\title{
International Poster Session
}

Ł印の演題は、学会の查読委員により優秀演題賞に選ばれた演題です。 民即の演題は、各学会の查読委員により選ばれた若手奨励賞受賞演題です。 凡 印の演題は、Travel Award Program 受賞演題です。

本プログラムに印刷されている演題は、インターネットによって応募された抄録内容が直接 印刷されております。そのため、表記の統一はなされておりません。(ごく一部において機械 的に変更されている場合もあります。)

*所属機関につきましては、演題登録締切後、所属機関へ正式名称の照会をさせていただき、 省略表記の統一を行いました。その結果、登録された名称と差異がある場合がございます。 ご了承ください。 


\section{Q IP-1_C}

Increased incidence of synchronus and metachronus esophageal cancer in patients with head and neck cancer in Taiwan

E-Da Hospital/School of Medicine, I-Shou University $\bigcirc$ Chao Ming Tseng, Ching-Tai Lee, Wen-Lun Wang

Background : Second primary esophageal squamous cell carcinoma (ESCC) frequently develop in the patients with head and neck cancer (HNSCC). This study aimed to examine the trend of incidence of synchronus and metachronus ESCC after diagnosis of HNSCC.

Methods : This retrospective population-based cohort study analyzed data from the Taiwan National Health Insurance Research Database from 1999 to 2013. A total of 68131 head and neck cancer patients were enrolled. Subjects with esophageal endoscopic screening (EE) within three months after the diagnosis of index HNSCC or without esophageal endoscopy (Non-EE), were matched $(1: 2)$ according to age gender Charlson comobidity index and index were analyzed using Cochran-Armitage trend test.

Results. The incidence of synchronous ESCC stepwise increased during the study period, from $0.0 \%$ in 1999 to $0.8 \%$ in 2012 ( $\mathrm{P}<$ $0.05)$. The Five-year and 10-year cumulative incidence rate of metachronous esophageal cancers were $1.4 \%$ and $2.7 \%$, respectively. in those with initial negative EE finding. Patients with oropharynx or hypopharynx cancers (high-risk) had significantly higher risk for developing metachronous esophageal cancers than those with developing metachronous esophageal cancers than those with ora or larynx cancers (low-risk) $(10-y c$
$\mathrm{HR}: 2.15 \quad[95 \% \mathrm{CI}: 1.57-2.96])$

Conclusion. The metachronous esophageal cancer continue to develop even at 10-year after treatment for primary HNSCC. Oropharynx and hypopharynx cancer survivors may require a longterm endoscopic surveillance

\section{IP-3_G}

Defined Lifestyle and Germline Factors Predispose Asian Populations to Gastric Cancer

Gastroenterology and Hepatology Dept. Yokohama City Univ., Sch. Med. ${ }^{1)}$, Genome Science Div. Rcast, Tokyo Univ. ${ }^{2)}$, Molecular Preventive Medicine Dept. Tokyo Univ., Sch. Med." ${ }^{3)}$ Pathology Dept. Tokyo Univ., Sch. Med."), Surgery Dept. Yokohama City Univ., Sch. Med. ${ }^{5)}$

OAkihiro Suzuki ${ }^{1.2)}$, Miwako Kakiuchi ${ }^{2)}$, Amane Tagashiraa ${ }^{2.4}$, Hiroto Kato ${ }^{3)}$, Daisuke Komura ${ }^{3)}$, Shogo Yamamoto ${ }^{2)}$, Kenji Tatsuno $^{2)}$, Eiji Sakai ${ }^{1)}$, Takashi Oshima ${ }^{5}$, Yasushi Rino, ${ }^{5)}$ Atsushi Nakajima $^{1)}$, Masashi Fukayama ${ }^{4)}$, Shumpei Ishikawa ${ }^{3)}$, Hiroyuki Aburatani $^{2)}$

Gastric cancer (GC) is one of the leading causes of cancer mortality throughout the world, with the highest incidence in east-Asia. Although the somatic genetics of GC has extensively been characterized by recent advances in cancer genome sequencing, the germline and environmental effects on GC and their ethnic differences have poorly been understood. Here, we performed genomic scale trans-ethnic analysis of 531 GC cases (319 Asian and 212 nonAsians), by integrating east-Asian and public GC datasets. There is one distinct GC subclass with clear alcohol-associated mutation signature and strong Asian specificity, and almost all the cases in the subclass are attributable to alcohol intake behavior and Asianspecific ALDH2 defective allele. Alcohol-related GCs have low mutation burden and a characteristic immune profile of increased Bcell infiltration into the tumor area in relation to cancer cell-intrinsic CXCL13 chemokine expression. In addition to germline variants of genes involved in BRCA pathways, we found frequent $(7.4 \%)$ germline CDH1 variants among Japanese GCs. Most of them were attributed to a few recurrent SNVs shared by both Japanese and Koreans, suggesting the existence of common ancestral events and widespread distributions of these pathogenic variants among eastAsian populations. These results revealed uncharacterized impacts of germline variants and their interplays with lifestyles in the high incidence areas.

gastric cancer

genome
Association between ALDH2 Polymorphism and Gastric Cancer Risk According to Alcohol Consumption : A Meta-Analysis

Department of Internal Medicine, Seoul National University Bundang Hospital ${ }^{1)}$, Department of Internal Medicine, Seoul National University Hospital Gangnam Center ${ }^{2}$, School of Public Health and Institute of Health and Environment, Seoul National University ${ }^{3)}$, Department of Internal Medicine and Liver Research Institute, Seoul National University College of Medicine ${ }^{4)}$

OCheol Min Shin ${ }^{1)}$, Seung Joo Kang's), Joohon Sung ${ }^{3)}$, Nayoung $\mathrm{Kim}^{4)}$

\section{Background}

Alcohol is thought to be associated with increased risk of gastric cancer. It has been suggested that alcohol is more carcinogenic in persons who possess the ALDH2 polymorphism.

\section{Methods}

We searched the PubMed, EMBASE, and Cochrane databases for relevant articles published between January 2000 and December 2018.

\section{Results}

Total 12 case-control studies consisting of 4,791 cases and 8,368 controls were included in the analysis. Inactive ALDH2 genotypes (G) A or A/A) were associated with an increased risk of gastric cancer $(\mathrm{OR}=1.21,95 \% \mathrm{CI}: 1.07-1.36)$ compared with active ALDH (G/G). Subgroup analysis by alcohol consumption showed more increased risk in moderate to heavy drinkers ( $\mathrm{OR}=1.83,95 \% \mathrm{CI}: 1.57-2.12$ ) than nondrinkers or mild drinkers $(\mathrm{OR}=1.16,95 \% \mathrm{CI}: 1.04-1.28)$. Alcohol consumption increased gastric cancer risk in persons who have inactive $A L D H \quad(O R=1.99,95 \% C I: 1.60-2.48)$ more than persons who have active ALDH (OR=1.48, 95\% CI : 1.13-1.94).

\section{Conclusions}

ALDH2 polymorphism may be associated with an increased risk of gastric cancer, especially in moderate to heavy drinkers. Gastric cancer is more susceptible among drinkers who have inactive ALDH.
All 4 markers were excellent diagnostic panels to clearly discriminate BE from CLE Moreover, as FABP1 and Hepar have different expression levels in dysplasia and EAC, these markers could function as key diagnostic aids in helping to determine the state of disease progression. 

Q $\mid P-5 \_E \quad \begin{aligned} & \text { Differences in biologic behaviors between } \\ & \text { mixed and pure signet ring cell carcinoma in }\end{aligned}$ Chinese early gastric Cancer patients

Changzhou No.2 People's Hospital

OXiaoyong Wang, Xiangrong Qin, Jia Wu

Background and Aim : The behavior of Signet ring cell carcinoma (SRC) carcinoma in early gastric cancer (EGC) is still controversial. This study aimed to compare clinicopathologic features between SRC and NSRC.

Methods : We reviewed medical records of patients with EGC between 2009 and 2014 in our hospital. We divided EGC into (1) pure SRC carcinoma, which was defined as composed only of signet ring cells (2) mixed SRC carcinoma, defined as signet ring cell carcinoma that was mixed with other histological components and (3) NSRC. We reviewed the clinicopathologic features.

Results : A total of 1008 patients were enrolled. 163 patients $(16.2 \%)$ were classified as SRC, including 63 cases of pure SRC and 100 mixed SRC. Stratification analyses according to the purity of SRC demonstrated that mixed SRC were associated with an increased risk of LVI $(\mathrm{P}=0.03)$ and LNM $(\mathrm{P}<0.001)$. In univariate analysis, LNM was associated with mixed SRC histologic type $(\mathrm{P}=$ $0.002)$, submucosal invasion $(\mathrm{P}<0.001)$ and the presence of LVI $(\mathrm{P}$ $<0.001)$. On multivariate logistic regression, the presence of LVI (odds ratio $26.70 ; 95 \%$ confidence interval 3.01-236.64; $\mathrm{P}=0.003$ ) and submucosal invasion (odds ratio $2.38 ; 95 \%$ confidence interval $1.01-5.60 ; \mathrm{P}=0.04$ ) were independent predictors of LNM in SRC.

Conclusion : Mixed SRC in EGC showed more aggressive behavior than other histologies.

early gastric cancer

signet ring cell carcinoma 


\section{IP-6_C}

Precision medicine of Gastric Cancer Patients Based on the PDX Model in NSG Mouse

The Affiliate Hospital of Qingdao University

OXiaoyu Li, Zibin Tian

Background : Gastric cancer (GC) is one of the most prevalent malignant cancers worldwide. Due to the relatively large inter-tumor heterogeneity, the precision medicine progression of GC is slow. Patient-derived xenografts (PDX) implant the patients'primary tumor tissues in immunodeficient mice which can predict patients tumor responses to anti-cancer agents. Our study aimed to establish GC PDX models and provide the optimal individualized therapeutic strategies. Methods : We implanted 30 cases of advanced GC tissue to the NSG mice and reported the tumor-forming period, traceability evaluation, and the immune cell infiltration. We also used a new method, Hollow Fiber Assay, to implant hollow fiber tubes under the skin of nude mice for rapid high-throughput drug screening in vivo. Results : The PDX models of GC in NSG mice were successfully established at least 4 months after three generations. Macrophages were dorminatedin the gastric adenocarcinomas PDX models, while the NK cells were dorminated in the gastric mucus adenocarcinomas. The hollow fiber assay significantly shortened the period of the implanted tumor growth and may inoculate 7 different kinds of tumor cells simultaneously. Conclusion : We established the GC PDX models successfully, and our results expanded the current understanding of the GC tumor microenvironment in PDX models. The hollow fiber assay could be used for the rapid highthroughput drug screening as a complement to the PDX models. IP-7_G $\begin{aligned} & \text { The Prognostic Effect of Immunohistochemical } \\ & \text { Stain of Biomarkers in Gastric Cancer }\end{aligned}$

St. Vincent's Hospital, College of Medicine, The Catholic University of Korea

OIk Hyun Jo, Woo Chul Chung, Yeon-Ji Kim, Jaeyoung Kim

Background and Aim : The treatment of gastric cancer is still unsatisfactory, and it has been known that few prognostic biomarkers are available but have limited values. We aimed to investigate the prognostic effect of various biomarkers - p53, EGRF, HER-2, Ecadherin, Ki-67- in patients with gastric cancer.

Methods : Between January 2014 and December 2016, we analyzed 505 gastric cancer tissues (279 early gastric cancers and 226 advanced gastric cancers) from the patients who had received radical gastric resection. All available immunohistochemical stained slides of surgical specimens for p53, EGRF, HER-2, E-cadherin, and Ki-67 were reviewed. We evaluated the various factors associated with disease recurrence during long-term follow-up.

Results : The median follow-up duration was 32.5 months (range : $7 \sim 70$ months). The higher levels of Ki-67 expression were in advanced gastric cancers, and otherwise were unremarkable. As for disease recurrence, lymphatic invasion and disease free interval, there was no prognostic effect of all biomarkers, except only HER-2 positive stage I gastric cancers. They had higher rate of disease recurrence $(P<0.01)$ and microscopic lymphatic invasion $(P=0.05)$.

Conclusions: The immunohistochemical activities for various biomarkers in prognosis of gastric cancer had limited values. Only, HER-2 expression could be a good biomarker for prognosis prediction for stage I gastric cancers.
IP-8_G

Analysis of mutation in early-stage diffuse-type gastric cancer with and without Helicobacter pylori infection through next-generation sequencing

Gastroenterology and Hepatology Center, Cancer Institute Hospital Ariake $^{1)}$, Cancer Institute, Cancer Precision Medicine Center ${ }^{2)}$ OKen Namikawa ${ }^{1)}$, Seiichi Mori ${ }^{2}$, Junko Fujisaki ${ }^{11}$

Background and Aim : The present study aims to identify more contributory mutations of the driver gene to cancer initiation through genome analysis in early-stage diffuse-type gastric cancer (EDGC) without Helicobacter pylori (HP) infection and discover DNA mutations related to DGC development through genome analysis in EDGC without HP infection. Furthermore, differences in mutation between HP-positive and HP-negative cancers were analyzed. Methods : Tissue samples were 26 lesions from 26 patients, including endoscopically or surgically resected formalin-fixed paraffin-embedded (FFPE) EDGC tissues and fresh frozen (FF) tissue procured as biopsy samples prior to resection, were analyzed. The size of DGC tumors should be $>10 \mathrm{~mm}$ in diameter. We classified tissues into two groups : (1) EDGCs without HP infection including $9 \mathrm{FF}$ and 5 FFPE samples and (2) EDGCs with HP infection including $9 \mathrm{FF}$ and $3 \mathrm{FFPE}$ samples. The EDGC tissues and paired blood samples were analyzed using whole-exome sequencing (WES). Result : CDH1 mutation was detected in 10 out of 14 without HP infection samples $(71.4 \%)$ and 7 out of 12 with HP infection samples $(58.3 \%)$. Conclusion: More CDH1 mutasions were detected in early-stage diffuse-type gastric cancers without Helicobacter pylori infection than with Helicobacter pylori infection, though the difference was not statistically significant.

\section{ASSESSMENT OF PD-L1 EXPRESSION IN SURGICALLY RESECTED \\ LYMPHOEPITHELIOMA-LIKE GASTRIC CARCINOMAS}

Department of Pathology, Jeju National University Hospital ${ }^{1}$, Department of Pathology, Asan Medical Center ${ }^{2}$ OSun Mi Lee ${ }^{1)}$, Chang Ohk Sung ${ }^{2)}$

Lymphoepithelioma-like carcinoma (LELC) is characterized by intra-or peritumoral immune cell infiltration, of which type may be a good candidate for immune checkpoint inhibitors. However, there has been no studies for assessment of PD-L1 expression in gastric LELCs. We investigated the PD-L1 status by immunohistochemistry in surgically resected gastric LELCs.

We included 200 surgically resected gastric LELCs consisting of 77 early gastric cancers and 123 advanced gastric cancers. Whole tumor sections of LELCs were assessed by immunohistochemistry for PD-L1 expression using E1L6N rabbit monoclonal antibody. The extent of immunoreactivity was graded according to the percentage of membranous staining of tumor cells and intensity of staining was graded as weak, moderate and strong.

95.5\% of LELCs showed variable PD-L1 expression including mild to strong labeling ranging from $5 \%$ to $80 \% .38 \%$ of LELCs revealed strong membranous labeling, which was seen in AGCs and submucosal LELCs. $20 \%$ of LELCs showed weak positivity with $5 \%$ or less. Within a whole tumor section, variable intensity of staining was observed, representing heterogeneity of PD-L1 expression in tumors.

LELCs showed significantly high expression of PD-L1 in tumor cells. PD-L1 overexpression was more frequently observed in advanced LELCs than early LELCs. High expression of PD-L1 in LELCs suggests that immune checkpoint inhibitors is a potential therapeutic modality for patients with advanced gastric LELC. 

with submucosal invasion

Department of Gastroenterology, Cancer Institute Hospital ${ }^{1)}$, Department of Pathology, Cancer Institute Hospital ${ }^{2)}$

OHiroki Osumi ${ }^{1)}$, Hiroshi Kawachi' ${ }^{2)}$, Toshiyuki Yoshio ${ }^{1)}$, Noriko Yamamoto $^{2)}$, Junko Fujisaki ${ }^{1)}$

Background : EBV positive gastric cancer (EBVGC) is associated with a low prevalence of LNM ; however, EBV status is not considered in the present indication of endoscopic resection (ER). We aimed to clarify the implication of EBV status for ER of pT1b GC. Methods : Consecutive cases of pT1b GCs treated with curative surgery between 2005 and 2014 were retrospectively analyzed. Tissue microarray was made and EBV-encoded RNA in situ hybridization was performed for evaluation of EBV status. Clinicopathological factors and LNM status were compared between EBVGC and non-EBVGC groups. Results : Among the 1221 pT1b GCs that underwent gastrectomy with regional lymph node dissection, 898 pT1 bGCs were eligible in this study. EBVGC accounted for $7.9 \%$ (71 of 898) cases. Compared to non-EBVGC, EBVGC was more frequent in males, the upper third region, showed elevated growth features, and was associated with a lower frequency of accompanying ulceration, greater depth of submucosal invasion, and lower frequency of lymphatic invasion. Frequency of LNM was significantly lower in EBVGC than in non-EBVGC (4.2\% vs. $21.9 \%$ ). In EBVGC, tumors without lymphovascular invasion showed significantly lower frequency of LNM than those with lymphovascular invasion ( 0 of 50 , $0 \%$; vs 3 of $21,14.3 \%$ ). Histologically, $84.5 \%$ (60 of 71 ) of EBVGC included carcinomas with lymphoid stroma and/or lace pattern components. Conclusion : pTlb EBVGC is a convincing candidate for $\mathrm{ER}$, regardless of risk factors other than lymphovascular invasion.

Epstein Barr virus pT1b gastric cancer 


\section{IP-11_G}

Preliminary investigation for the life characteristics of gastro-oesophageal reflux disease and functional dyspepsia patients in southern China

Department of Gastroenterology and Hepatology, Guangdong Provincial People's Hospital/Guangdong Academic of Medical Science/ Guangdong Provincial Geriatrics Institute

OZhongwen Zheng, Xiaoling Bu, Weihong Sha, Aiping Shen, Dongli Wang, Fulong Luo

Background Gastro-oesophageal reflux disease (GORD) and functional dyspepsia (FD) are common digestive diseases. The difference of life characteristics between GORD and FD is little known. Objectives This study aims to investigate living characteristics of GORD, FD patients in the southern China by analyzing basic information, life habits and quality of life score. Methods 48 GORD patients, $27 \mathrm{FD}$ patients were enrolled and general information, living features, quality of life score (includes health-transition (HT), physical functioning (PF), role-physical (RP), bodily pain (BP), general health $(\mathrm{GH})$, vitality $(\mathrm{VT})$, social functioning $(\mathrm{SF})$, role-emotional (RE), mental health $(\mathrm{MH})$ ) were analyzed. Results Between GORD and FD groups, difference appeared on eating habits (12.5\% vs $29.6 \%, \mathrm{p}=0.048)$, regular defecation $(35.4 \%$ vs $63.0 \%, \mathrm{p}=0.021)$, normal stool frequency $(75.0 \%$ vs $92.6 \%, \mathrm{p}=0.041)$, sports hobby $(37.5 \%$ vs $14.8 \%, \mathrm{p}=0.036)$, $\mathrm{BP}(63.8$ vs 74.8 scores, $\mathrm{p}=0.029)$, SF $(72.7$ vs 63.4 scores, $\mathrm{p}=0.043$ ) and siesta time (45.4 vs 27.5 minutes after lunch, $\mathrm{p}=0.046)$. No difference was found on other living characteristics and scores. Conclusions FD patients tends to suffer more bodily pain and worse social activities. GORD patients seems needing more digestion time before siesta. More abnormal eating habits and less physical exercise are common in FD patients, while GORD patients experience higher irregular defecation rate and abnormal stool frequency. Deeper study is needed to understand the relationship between GORD, FD and life characteristics.

Gastro-oesophageal reflux disease Functional dyspepsia

\section{\&IP-12_G}

Clinical analysis of esophagogastric junction outflow obstruction

Department of Internal Medicine, Gangnam Severance Hospital, Yonsei University College of Medicine ${ }^{1}$, Department of Internal Medicine, Yonsei University College of Medicine ${ }^{2)}$

OYoung Min Kim¹), Jeung Eun Lee ${ }^{2)}$, Da Hyun Jung ${ }^{1)}$, Jun Chul Park $^{2)}$, Jie-Hyun Kim ${ }^{1)}$, Young Hoon Youn ${ }^{1)}$, Hyojin Park ${ }^{1)}$

\section{Background/Aim}

There were no significant clinical characteristics and consensus treatment guideline regarding esophagogastric junction (EGJ) out-
flow obstruction. The aim of our study was to investigate clinical significance of this rare disease.

\section{Methods}

We retrospectively reviewed electronic medical records of $2,684 \mathrm{pa}$ tients who underwent high-resolution manometry (HRM) between January 2011 and January 2018. EGJ outflow obstruction was diagnosed by HRM characterized by elevated relaxation pressure (IRP) and intact peristalsis. We analyzed HRM parameters and clinical course of patients.

\section{Results}

A total of 53 patients were diagnosed with primary EGJ outflow obstruction. Most patients $(75.0 \%)$ presented with dysphagia or ches pain. HRM parameters (mean \pm standard deviation) were $39.0 \pm 13.6$ $\mathrm{mmHg}$ in basal lower esophageal sphincter pressure and $20.5 \pm 9.57$ $\mathrm{mmHg}$ in IRP. 10 patients received endoscopic treatment. 1 patient $(100 \%)$ after POEM, 5 patients $(80 \%)$ after Botox injections, and 4 patients $(50 \%)$ after pneumatic dilatation improved symptoms. Among 6 patients who underwent follow-up HRM, 2 patients developed achalasia.

\section{Conclusions}

Primary EGJ outflow obstruction patients presented dysphagia or chest pain dominantly. Endoscopic treatments showed improvement of symptoms. In follow-up HRM, some patients developed achalasia. However, further research with large scale is warranted
to clarify clinical significance of this data.

\section{Q}

URGENT ENDOSCOPY IS NOT ASSOCIATED WITH LOWER MORTALITY IN HEMODYNAMICALLY UNSTABLE UPPER GASTROINTESTINAL BLEEDING

Division of Gastroenterology, Department of Medicine, Siriraj Hospital Mahidol University

OUayporn Kaosombatwattana, Manassawee Chakpaisan, Julajak Limsrivilai, Nonthalee Pausawasdi, Phunchai Charatcharoenwitthaya, Somchai Leelakusolvong

Background/Aims : Role of very early endoscopy in patients with acute UGIB remains debatable. We aimed to compare the results of timing of endoscopy in stable versus unstable hemodynamics at presentation. Methods : This prospective observational study included UGIB patients who underwent endoscopy between 20112017. The 30-day mortality and rebleeding rate were analyzed in two subgroups regarding hemodynamic status. Hemodynamic instability was defined as SBP $<100 \mathrm{mmHg}$ and/or HR $>100$ beats/ min. Result : 1,359 patients were included, 458 (35.7\%) were hemodynamically unstable. Urgent endoscopy increased rebleeding rate and 30-day mortality in patients with hemodynamic instability whereas it did not affect clinical outcomes those with stable hemodynamics. Conclusion : Urgent endoscopy did not provide mortality benefit and may cause harm, underscoring the notion by international guidelines that it should be offered to selected cases after adequate resuscitation.

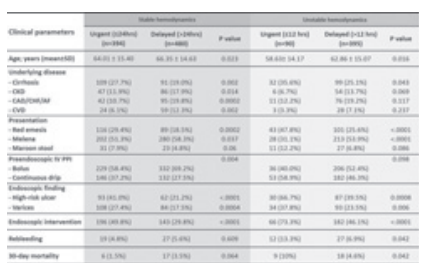

upper gastrointestinal bleeding

timing of endoscopy

IP-14_G

Tailored vonoprazan-based first line eradication therapy of Helicobacter pylori optimized by clarithromycin susceptibility testing

Department of Gastroenterology and Hepatology, Numazu Municipal Hospital

ODaisuke Miyagishima, Naoto Fujita, Kaoru Sugimura, Hiromasa Suzuki, Akane Kurosugi, Shingo Kasamatsu, Michio Kubota, Akihiko Nakagawa, Masami Shinozaki

Background : Vonoprazan (VPZ) -based first-line Helicobacter pylori eradication therapy optimized by clarithromycin (CAM) susceptibility testing has been recommended by JSHR in 2016, but there are few published data in clinical practice. We aimed to compare effectiveness of tailored VPZ-based first-line therapy with conventional VPZ-based therapy.

Methods : Patients who underwent first-line eradication therapy and received judging examination in our hospital between April 2016 and April 2018 were enrolled. Eligible patients were divided into a conventional group (VPZ $20 \mathrm{mg}$, amoxicillin $750 \mathrm{mg}$, CAM 200 $\mathrm{mg}$, twice daily, for 7 days without susceptibility test) and a tailored group (CAM 200mg or metronidazole (MNZ) 250mg was chosen according to CAM-susceptibility results according to JSHR guideline 2016), and eradication success rate was compared.

Results : A total of 212 patients were enrolled in this study. The tailored group included 108 subjects and the conventional group included 104 subjects. Eradication success rate was 95.4\% (103) 108) for tailored group and $90.4 \%$ (94/104) for conventional group. The eradication rate in tailored group was higher than conventional group, but there was no significant differences between the two groups $(p=0.157)$. The prevalence of resistance to clarithromycin (MIC $\geq 1 \mu \mathrm{g} / \mathrm{ml})$ was $17.8 \%$.

Conclusions : Tailored first-line VPZ-based eradication therapy of $H$ pylori optimized by clarithromycin susceptibility testing is the most effective eradication strategy. 
IP-15_G 演題取り消し 


\section{\&IP-16_G}

Preliminary discussion of the clinical significance for lactase deficiency and selfreported lactose intolerance symptoms

Guangdong Provincial People's Hospital

$\bigcirc$ Chen Zhang, Wen Zhong Zheng, Ling Xiao Bu, Hong Wei

Sha, Chao Bo He, Ping Ai Shen, Li Dong Wang

【Abstract】Background Lactase Deficiency (LD) is a common phenomenon in China which is commonly diagnosed by lactose hydrogen breath test (LHBT). Some people have self-reported lactose intolerance (LI) symptoms after lactose consumption. However, the clinical significance of LD and self-reported LI symptoms are still not clear. Objectives This study aimed to analyze whether the severity degree of LD and the self-reported LI symptoms presented the clinical significance. Methods 84 outpatients from the past three years were enrolled in this study. The clinical features of these subjects including age, sex, body mass index (BMI) were recorded. All the subjects accepted LHBT, gastrointestinal endoscope, allergen tests and routine tests (blood routine test, liver and renal function test et.). According to the LHBT results, people were graded as "negative LD (NLD), mild LD (MILD), moderate LD (MOLD) and severe LD (SLD)". For further analysis, subjects were divided into two groups : self-reported LI symptoms positive and symptoms negative. The analysis was performed between clinical parameters among each group. Results No difference was found on sex, average age, BMI, the rate of allergen, the rate of self-reported LI symptoms and other clinical parameters among NLD, MILD, MOLD and MOLD. Compared with self-reported LI symptoms negative group, the positive group presented lower trends on nutrition index including BMI ( $\mathrm{P}=0.019,95 \% \mathrm{CI},-4.44 \sim-40)$, Serum Total Protein ( $\mathrm{P}$ $=0.033,95 \%$ CI,-7.09 -.29), Albumin $(\mathrm{P}=0.041,95 \% \mathrm{CI},-4.70 \sim-.09)$. Conclusions The degree of LD plays a limited role in guiding clinical treatment.Patients who have self-reported LI symptoms seem to have lower nutrition level.

Lactase deficiency

clinical significance

\section{IP-17_G}

Enhancement of long-term relapse-free rate by infliximab and plant-based diet as first-line (IPF) therapy in Crohn disease

Akita City Hospital

$\bigcirc$ Mitsuro Chiba, Tsuyotoshi Tsuji, Yu Obara, Hideo Ohno, Hajime Ishii, Satoko Tsuda, Kunio Nakane, Masafumi Komatsu

BACKGROUND : The lifelong relapse-free rate in Crohn disease (CD) is $10-15 \%$. The most ubiquitous environmental factor in inflammatory bowel disease is westernized diet (Perm J 2019;23: 18-107). The relapse preventive effect of plant-based diet (PBD) was reported (World J Gastroenterol 2010 ; $16: 2484$ ) and successful induction without primary nonresponders was reported by infliximab and PBD as first-line (IPF) therapy in CD (Perm J 2017;21:17009).

AIM : To investigate whether IPF therapy enhances long-term relapse-free rates in CD.

METHODS : Twenty-four remitted adult patients treated by IPF therapy were included. At discharge, patients were advised to adhere to PBD. Biologics were not used. Relapse was defined as appearance of symptoms of $\mathrm{CD}$ resulting in alteration of therapeutic modality. PBD score (PBDS), which evaluates adherence to PBD was calculated three times : before IPF therapy (baseline), 2 years after discharge (short-term), and beyond 2 years (long-term). A higher PBDS indicates greater adherence to PBD.

RESULTS : The cumulative relapse-free rate by Kaplan-Meier at 1, 2,3 , and 4 years was $79 \%, 66 \%, 57 \%$, and $52 \%$, respectively. There was no further reduction afterwards. Mean short- and long-term PBDS were 30.7 and 24.0 , respectively, which were significantly higher than baseline PBDS (9.2).

CONCLUSION : The relapse-free rate at 5 years after IPF therapy was $52 \%$. This indicates IPF therapy can remarkably enhance the long-term relapse-free rate.

\section{IP-18_G}

Clinical outcome with Ustekinumab in medically-refractory Crohn's disease : real world experience from a single center cohort

Inflammatory Bowel Disease Center, Fukuoka University Chikushi Hospital $^{1}$, Department of Gastroenterology, Fukuoka University Chikushi Hospital ${ }^{2)}$

ONoritaka Takatsu ${ }^{12}$, Teruyuki Takeda ${ }^{1)}$, Masahiro Kishi ${ }^{2}$,

Takashi Hisabe ${ }^{2)}$, Kenshi $\mathrm{Yao}^{2)}$, Toshiharu Ueki ${ }^{2)}$

Background and purpose : Few studies have described the real world experience with ustekinumab (UST) in patients with Crohn's disease $(\mathrm{CD})$. We aim to describe the real world clinical outcomes achieved with UST in a single center cohort of CD

Methods: The subjects were CD patients who received UST at our hospital. The primary endpoints were achievement of clinical response and remission rate at 8 and 52 weeks. The secondary endpoint was treatment safety. Clinical response was defined as a decrease from baseline in CDAI score of at least 100 points or a total CDAI score less than 150 . Clinical remission was defined as a total CDAI score $<150$.

Results : Twenty-eight patients were treated with UST. The subjects comprised 14 males with a median age of 45.5. The median CDAI was 199. As for the reasons for introduction of UST, 25 patients were failure or intolerance to anti-TNF $\alpha$. At 8 weeks, clinical response was achieved in $62 \%$ and remission in $58 \%$. At 52 weeks, clinical response was achieved in $75 \%$ and the remission rate was the same number. There were not serious adverse events that were necessity of UST cessation.

Conclusion : UST is an effective and safe therapeutic option for inducing and maintaining clinical remission in patients with CD failing anti-TNF $\alpha$.

ustekinumab

Crohn's disease
Living with ulcerative colitis in Japan : Biologic persistence and healthcare resource utilization

Pfizer Inc, New York, USA ${ }^{1)}$, Adelphi Real World ${ }^{2)}$, Pfizer Inc, Groton, CT, USA ${ }^{3)}$, Pfizer Inc, Madrid, Spain ${ }^{4)}$, Pfizer Inc, Tokyo, $\mathrm{Japan}^{5)}$, Kitasato University Kitasato Institute Hospital ${ }^{6}$ ODanielle Bargo ${ }^{1)}$, Theo Tritton ${ }^{2}$, Joseph Cappelleri' ${ }^{3)}$, Irene Modesto $^{4)}$, Marco DiBonaventura ${ }^{1)}$, Timothy Smith ${ }^{1}$, Takanori Tsuchiya ${ }^{5)}$, Sean Gardiner ${ }^{5)}$, Stuart Blackburn ${ }^{2)}$, Taku Kobay$\operatorname{ashi}^{6)}$

Aims : Describe persistence to first biologic therapy and establish the associated healthcare costs among patients with ulcerative colitis (UC).

Methods : Retrospective longitudinal cohort design indexing UC patients on initiation of first biologic therapy between Aug-2013 and Jul-2016 with 2-year follow-up to assess resource-use, utilizing Japanese hospital administrative/claims data (MDV). Patients stratified on persistence to indexed biologic at 6/12/18-months.

Results : Biologic therapy persistence was $66.9 \%$ : 6-months, $58.1 \%$ : 12-months, $51.8 \%$ : 18-months. Approximately $70 \%$ of nonpersistent patients were not treated with biologics following discontinuation at each timepoint. Non-persistence was associated with lower prescription costs, but greater inpatient costs and time-incare (12-months : 19.6 vs. 4.5 days,p $<0.0001)$.

Conclusion : Observed low levels of persistence to biologic therapy may be associated with increased burden on inpatient healthcare services.

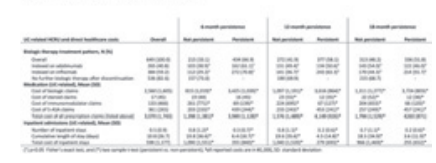

Ulcerative Colitis 

LPA 1 -20_G TL1A-DR3 system in naive T cells leads

Department of Gastroenterology and Neurology, Akita University Graduate School of Medicine

OYosuke Shimodaira

【Background】 TL1A is an IBD susceptibility gene. DR3, the receptor for TL1A, is expressed on T cells, fibroblasts and ILC2 and its engagement can lead to apoptosis, proliferation and activation. The role of TL1A and DR3 to the development of chronic colitis is largely unknown. 【Aim】 To determine the contributions of TL1A and DR3 in different cell types in adoptive transfer colitis. 【Method】 Naive T cells isolated from WT, Tlla-/-, or Dr3-/- mice were transferred into Rag-/-, Rag-/-Tlla-/-, or Rag-/-Dr3-/-. Weight was monitored and colitis was assessed by H\&E. IFN $\gamma$ - and IL17producing $\mathrm{T}$ cells in the intestinal mucosa and MLN was determined by flow cytometry. For in vitro $\mathrm{T}$ cell differentiation assays, naive T cells from WT, Tlla-/- or Dr3-/- were differentiated into Th 1 and Th17 cells. [Result]Rag-/-Tlla-/- mice receiving Tlla-/-naive $\mathrm{T}$ cells experienced less weight loss compared to Rag-/- receiving WT T cells. Protection from colitis development was also reflected by reduced histoscores in Rag-/-Tlla-/- mice receiving Tlla-/- T cells, as well as by reduced numbers of IFN $\gamma+\mathrm{CD} 4+\mathrm{T}$ cells in the mucosa and MLN. Number of IL17+CD4+T cells was not different in these mice. TL1A-deficiency in donors ( $\mathrm{T}$ cells) alone recapitulated this phenotype but TL1A-deficiency in recipients (all non-T or non-B cells) alone did not. Rag-/-Dr3-/- receiving Dr3-/- T cells had reduced numbers of IFN $\gamma+\mathrm{CD} 4+\mathrm{T}$ cells in the mucosa and MLN. In vitro differentiation into Th1 cells was impaired in Tlla-/- and Dr 3-/- cells but Th17 differentiation was unchanged. 【Conclusion】Our data indicate that TL1A contributes to chronic T cell driven colitis by effecting Thl differentiation in vivo. 
IP-21_G

The use of rifaximin in the treatment of incomplete bowel obstruction

Digestive Department, Kiang Wu Hospital

$\bigcirc$ Hon $\mathrm{Ho} \mathrm{Yu}^{1)}$, Chin Wang Chiang ${ }^{2)}$, Jin Wen Zhang ${ }^{2)}$

\section{Background :}

The use of rifaximin in incomplete bowel obstruction management provided good treatment outcome which may due to the local ef fects on GI flora.

\section{Case presentation :}

77 years old female with history of hypertension and cerebral em-
bolism presented in hospital on April 2016. She had blood stools with constipation for one year and abdominal pain for four months prior admission. The patient was diagnosed with sigmoid tumour and followed by sigmoid colectomy and appendectomy. Pathological report suggests intermediate grade of Adenocarcinoma of the Copost-surgery. However, rifaximin was commenced on 27 May and the symptoms of abdominal discomfort and bloating was improved Symptoms were continuously improved and rifaximin was ceased on 20 June.

\section{Conclusions :}

The patient presented bloating and vomiting post-surgery. How ever, rifaximin was shown to provide symptomatic relief and facilitates the recovery process. it is concluded that rifaximin not only bowel obstruction management.

Medication chart :

3 days post op
Duphalac SYR 6Oml

biofermin-s $1 \mathrm{td} 20 \mathrm{ml}$ bd
PARAFFIN LIQ $30 \mathrm{ml}$ bd

6 days post op

Xifaxan 200mg bd

16 days post op
Xifaxan $200 \mathrm{mg}$ bdX 10 days after $\mathrm{D} / \mathrm{C}$

28 days post op

combizym 1 bds, dogmatil 50mg bds

rifaximin

incomplete bowel obstruction

\section{IP-22_G}

Altered Intestinal Permeability and Effect of Mosapride on Postoperative Ileus in a Guinea Pig Model

Department of Internal Medicine, Gangnam Severance Hospital, Yonsei University College of Medicine

OYoung Min Kim, Zahid Hussain, Young Ju Lee, Hyojin Park

\section{Background/Aims}

The alteration of permeability and accumulation of inflammation seems to play an important role in the pathophysiology of Postoperative ileus (POI). This study was performed to characterize the alteration of intestinal permeability regarding the development of POI. Moreover, we investigated whether mosapride, a 5-HT4 recepPOI. Moreover, we investigated whether mosapride, a 5-HT4 recep-
tor agonist, has an effect on the intestinal permeability and has an tor agonist, has an effect on the intestinal
anti-inflammatory effect in the POI model.

\section{Methods}

An experimental POI model with guinea pig was created. Fluorescent markers (FITC-dextran) were passed through the harvested intestinal membranes in the Ussing chamber and checked for differences in optical translucency in the ileum and the proximal colon. We compared intestinal permeability and inflammatory grade represented by leukocyte counts and the expression of calprotectin between control group, POI group, and group of mosapride $(0.3 \mathrm{mg} / \mathrm{kg}$ and $1 \mathrm{mg} / \mathrm{kg}$, s.c.) administration after surgical manipulation.

\section{Results}

The ileum and proximal colon showed increased permeability and inflammatory grade in the POI group compared with control group. Increased permeability of POI group was significantly decreased af ter mosapride administration. However, elevated leukocyte counts and the expression of calprotectin of POI group was no significant change by mosapride.

\section{Conclusion}

Increased intestinal permeability and inflammatory grade were observed in the POI guinea pig model. Mosapride has a reducing effect on this increased permeability.
201P-23_G

CT colonography vs colonoscopy for the diagnosis of colorectal cancer : a cost utility analysis from a United Kingdom health system perspective

Imperial College School of Medicine ${ }^{1)}$, Imperial College Business $\mathrm{School}^{2)}$

OAnam Malik ${ }^{1)}$, Olaoluwamide Akinwuntan ${ }^{1)}$, Sunnyath $\mathrm{Ali}^{2)}$, Jenanan Mohan ${ }^{1)}$, Falak Naqvi ${ }^{1)}$, Harmanjit Singh ${ }^{1)}$, Konstantinos Tourmouzis ${ }^{2}$, Laure De Preux ${ }^{2)}$

Background

While colonoscopy is considered gold standard for colorectal cancer (CRC) diagnosis, literature suggests newer non-invasive methods lar sensitivity to colonoscopy, lower cost and lower risks of complications. This study aimed to investigate the cost utility of CTC against coctive.

Method

A literature review was performed to obtain lifetime utility 2019) and values were plotted on a decision tree Using this an incremental cost-effectiveness ratio (ICER) was calculated.

Results Calculations revealed CTC to cost $£ 2752 / 0.798$ QALY whereas
colonoscopy costing $£ 1975 / 0.835$ QALY. An ICER value of $£-$
$20895.581 /$ QALY indicates that CTC is less cost effective that 20895.581 /QALY indicates that CTC is less cost effective that
colonoscopy. One-way sensitivity analysis reducing the probability colonoscopy. One-way sensitivity analysis reducing the probability
of inconclusive results following CTC showed an ICER of $£ 102$ of inconclusive results following CTC show
829 / QALY, making CTC more cost effective.

Discussion

There is a higher probability of inconclusive results after CTC leading to further investigations being needed (due to a lack of stanscopy currently remains more cost effective, widespread use of CTC in future years leading to nationwide training of radiologists may lower inconclusive results and therefore further research is recolorectal cancer CT colonography

IP-24_G

The Diagnostic Accuracy of Papanicolau Staining in Brush Biopsy as an Alternative to Tissue Biopsy in Patients Undergoing Colonoscopy for Non-Obstructing Colonic Tumors

Internal Medicine-Section of Gastroenterology, VSMMC

$\bigcirc$ Clint Lumba Becaldo, Arlene Matute Kuan, Leslee Yap Ong

Introduction : In the Philippines in 2010, colorectal cancer combined rank $4^{\text {th }}$ for both sexes. Early detection of colorectal malignancy remains the forefront of long-term management.

Objective : The study was conducted to determine the diagnostic accuracy of papanicolau stain in detecting malignancy from brush biopsy specimens in patients undergoing colonoscopy.

Methodology : A prospective, open label 19-month study of patients aged 19 years and above. Demographics and informed consent of all patients undergoing colonoscopy were taken. On colonoscopy, brush biopsies around the four sides of tumors were sampled after the tissue biopsy then smeared to slides, fixed with $95 \%$ ethyl alcohol and stained with Papanicolau method and read by 2 independent pathologists.

Results : 60 patients underwent colonoscopy for colonic tumors. Average age was 55.60 years old, $53.3 \%$ were females, $85 \%$ had family history of colon cancer and $50 \%$ were smokers. A significant association of Pap stain in detecting malignancy $(\mathrm{p}=0.002)$ was noted with sensitivity of $79.2 \%$ and specificity of $66.6 \%$.

Conclusion : The result of this study showed good sensitivity of using Pap stain in that it is able to correctly identify $79.2 \%$ for patients who truly have colon cancer with specificity of $66.6 \%$. A positive Pap stain is highly associated with colon cancer detection (PPV $=90.5 \%$ ).

colorectal cancer

papanicolau stain 


\section{\& IP-25_G Quality of Life After Colorectal Cancer}

University Surgical Cluster, National University Health System ${ }^{11}$, Yong Loo Lin School of Medicine, National University of Singapore ${ }^{2)}$ OHeidi SY Chang ${ }^{1)}$, Tian Zhi Lim', Ning Qi Pang ${ }^{1)}$, Dedrick Chan $^{1)}$, Ker-Kan Tan ${ }^{1.2)}$

\section{Purpose}

Quality of life (QoL) is an important aspect in the management of colorectal cancer. We aim to evaluate the QOL of colorectal cancer patients after diagnosis and/or treatment.

\section{Methodology}

A cross-sectional study was conducted on 100 consecutive patients who presented with a history of colorectal cancer, who had undergone treatment. Patients were administered the EORTC QLQ-C30 questionnaire. The Global Health Status of the questionnaire, which measures QoL, was scored between 0 to 100, where 100 denotes perfect QoL.

\section{Results}

The median age was $65(27-87)$. The mean QoL score of our study population is $68.0 \pm 23.6$. Stoma creation had no impact on the QoL There was no difference in QoL in patients with stoma created in the index operation $(65.0 \pm 24.8)$ compared to those without (64.8 $\pm 23.6)$. Stoma reversal also did not have a significant effect on the QoL $(60.7 \pm 21.4$ vs $66.7 \pm 26.1)$. Comparing the QoL of patients who underwent chemo/radiotherapy (RT) against patients who never had chemo/RT, no significant difference was noted $(64.0 \pm 22.3$ vs $70.0 \pm 24.5)$. Amongst those who underwent resection, chemo/RT did not result in a significantly different QoL compared to those without $(66.7 \pm 24.7$ vs $71.7 \pm 22.3)$.

\section{Conclusion}

QoL is important in the treatment of patients with colorectal cancer. Stoma and chemo/RT may not have as large an impact on QoL as previously thought.

Colorectal Cancer

Quality of Life 


\section{IP-26_G}

The potency to halt hepatocellular carcinoma and colorectal cancer progression as cancer immunotherapy to suppress MICA release

Division of Gastroenterology, Department of Medicine, Showa University School of Medicine ${ }^{1)}$, Department of Gastroenterology, Graduate School of Medicine, Chiba University ${ }^{2)}$

OJun Arai $^{1)}$, Ryo Nakagawa ${ }^{2)}$, Ryosuke Muroyama ${ }^{2)}$, Yumi Otoyama $^{1)}$, Ikuya Sugiura ${ }^{1)}$, Yoko Nakajima ${ }^{1)}$, Atsushi Kajiwara $^{1)}$, Yuki Ichikawa ${ }^{1)}$, Shojiro Uozumi ${ }^{1)}$, Yuu Shimozuma ${ }^{1)}$, Manabu Uchikoshi ${ }^{1)}$, Masashi Sakaki ${ }^{1)}$, Naoya Kato ${ }^{2)}$, Hitoshi Yoshida $^{1)}$

$<$ Background $>$ Regorafenib (REG) is approved for hepatocellular carcinoma (HCC) patients who got resistant to sorafenib (SOR) as well as colorectal cancer (CRC). In our previous GWAS study, MHC class I-related chain A (MICA) was correlated with HCC development (Nat Genet 2011). MICA is a cell-bound NKG2D ligand and shed into the sera of patients as soluble MICA (sMICA), being immunological decoy for NK cells. We have shown REG decreases sMICA of HCC more than SOR, inducing NK cell-mediated cytotoxicity (J Gastroenterol Hepatol 2018). Here the influence of REG to sMICA in CRC is examined. The sheddase of MICA in CRC is also evaluated.

$<$ Methods >1) Human CRC cells (HCT116 and HT29) were treated with REG at $0.5,1$, and $2 \mu \mathrm{M}$ to measure the sMICA level by ELISA. 2) Potential candidates to shed MICA were knocked down with siRNA.

$<$ Results $>1$ ) REG significantly decreases sMICA in a dose dependent manner $(\mathrm{P}<0.001$ at 1 , and $2 \mu \mathrm{M})$.2) Knockdown of ADAM17, not ADAM9, 10, or VEGFA, in HT29 significantly decreases SMICA.

$<$ Conclusion $>$ Suppression of sMICA release by REG could provoke NK cell-mediated cytotoxicity. Interestingly, ADAM17 correlates with MICA shedding, being a potent druggable target for the CRC patients.

\section{Q}

IP-28_G

Predictive Factors for Risk of HCC in Immune Inactive $\mathrm{CHB}$

Kangdong Sacred Heart Hospital of Hallym University Medical Center

OHyoung Su Kim, Myoung Kuk Jang, Woon Geon Shin, Hak Yang Kim, Choong Kee Park

BACKGROUND \& AIMS : The risk factors for hepatocellular carcinoma (HCC) in immune inactive chronic hepatitis B (CHB) have not been clarified. The aim of this study was to investigate the predictive factors for $\mathrm{HCC}$ in immune inactive $\mathrm{CHB}$.

METHODS : A total of 337 patients with immune inactive CHB were enrolled and analyses were performed to identify the risk factors for HCC.

RESULTS : During the mean 63 months, the incidence of HCC in the total study population was $4.5 \%$. Patients who developed HCC were older, had more cirrhosis, and were more likely to experience ALT elevation $>2 \mathrm{X}$ upper limit of normal (ULN) during follow-up than those without HCC. In Cox regression analysis, increased ALT levels $>2 \mathrm{X}$ ULN during follow-up and presence of cirrhosis were identified as the independent factors. With increasing number of risk factors, the respective cumulative incidence of $\mathrm{HCC}$ at 10 years was $6.3 \%, 8.8 \%$, and $63.5 \%$.

CONCLUSIONS : Underlying cirrhosis and hepatic inflammation reflected by increased ALT levels $>2 \mathrm{X}$ ULN were significant predictors for HCC in immune inactive CHB. ALT elevation showed a synergistic effect in HCC development combined with cirrhosis. It suggests that patients with high serum ALT levels, especially those with cirrhosis, require closer surveillance for $\mathrm{HCC}$ even in immune inactive $\mathrm{CHB}$.

chronic hepatitis B

Hpatocellular carcinoma

IP-29_G

Clinical implication of circulating tumor cellsin patients with hepatocellular carcinoma using a microcavity array

Second Department of Internal Medicine, University of Fukui OKazuto Takahashi, Kazuya Ofuji, Katsushi Hiramatsu, Takuto Nosaka, Tatsushi Naito, Hidetaka Matsuda, Masahiro Ohtani, Tomoyuki Nemoto, Yasunari Nakamoto

Purpose : The aim of this study was to evaluate the clinical significance of circulating tumor cells (CTCs) in patients with chronic liver disease (CLD) and hepatocellular carcinoma (HCC) using a microcavity array (MCA), which was a novel isolation system for CTCs.

Experimental Design: Peripheral blood samples $(3 \mathrm{~mL})$ were processed using the MCA system from 7 healthy donors, 14 patients with liver cirrhosis and 31 patients with HCC. We investigated mRNA expression of liver-specific genes in isolated CTCs using $\mathrm{qPCR}$.

Results : In samples from HCC patients, positivity rate was $90.3 \%$ and the mean number of detected CTCs was 47.6 \pm 114.1 (mean \pm $\mathrm{SD})$. The number of CTCs was significantly higher in patients with metastatic HCC $(102.2 \pm 160.6)$ than in those with localized HCC (8.2 $\pm 7.7)(\mathrm{P}<0.05)$. Expression of AFP, glypican-3, EpCAM and albumin genes was detected in isolated CTCs. The cumulative survival of patients with $\geq 10$ CTCs or ALB-positive patients in mRNA expression of CTCs was significantly reduced $(\mathrm{P}=0.025, \mathrm{P}=0.037$, respectively). Furthermore, we found that it was the CTC counts as well as tumor number and tumor size that correlated with patients with metastatic HCC.

Conclusion : The MCA system may provide a predictive tool for prognosis in HCC patients and a new strategy to analyze mRNA expression of CTCs for precision medicine. 
IP_30 G Clinical and biological significance of BMP9 in the malignant nature of hepatocellular carcinoma

Department of Gastroenterology, Kanazawa University Hospital OKouki Nio, Taro Yamashita, Shuichi Kaneko

Background : The major malignant phenotypes of liver cancer are related to the presence of cancer stem cells (CSCs). We recently reported that distinctive CSCs regulating tumorigenicity (EpCAM+) and metastasis $(\mathrm{CD} 90+)$ have different epithelial/mesenchymal gene expression signatures. BMP9 has recently been highlighted as a molecule involved in the proliferation of hepatocellular carcinoma (HCC) cells. Here, we investigated the clinical and biological significance of BMP9 in the malignant nature of HCC.

Methods : BMP9 expression in human HCC tissue and in HCC patient serum were examined by IHC and ELISA, respectively, and correlations with prognosis and recurrence were analyzed. The effects of BMP9 on CSC maintenance and angiogenesis were examined using HCC cell lines and HUVEC.

Results : In IHC cohort, patients with BMP9-high HCC had a poorer prognosis and more advanced form of recurrence than patients with BMP9-low HCC. Similarly, patients with serum BMP9high had a poor prognosis. In HCC cells, BMP9 increased EpCAMpositive liver CSC subsets and the expression of EpCAM gene as well as BMP9 signaling genes, including inhibitors of DNA binding 1 (ID1). Interestingly, BMP9-induced EpCAM gene expression was reduced by ID1 knockdown. In addition, BMP9 activated tube formation in HUVECs.

Conclusion: These findings suggests that BMP9 is a novel biomarker of $\mathrm{HCC}$ and affects the malignant nature of $\mathrm{HCC}$ through CSC maintenance and angiogenesis. Thus, BMP9 signaling could be a pivotal therapeutic target against HCC.

BMP9

liver cancer stem cell 


\section{Q RIP-31_E}

Video capsule endoscopy in Pediatric population : Single-center experience of 15 year

University Hospital Virgen Macarena

OVictoria Alejandra Jimenez-Garcia, Federico Arguelles-Arias,

Rafael Romero-Castro, Laura Lorenzo-Gonzalez, Carmen Cuadrado, Pedro Hergueta-Delgado, Angel Caunedo-Alvarez

Background : Study of small bowel pathology in the pediatric age is complex due to the invasiveness of most of the available tests. Video capsule endoscopy (VCE) in children has some limitations based on age. However, is a non-invasive useful diagnostic procedure.

Aims : We retrospectively reviewed the VCE procedures in pediatrics performed in an academic single-center, focused on indications, findings, safety and diagnostic yield.

Results : Since September 2001 to January 2019, 120 VCE procedures were performed in pediatrics (3-14 years-old). The main indications and findings are in Table 1 . Adverse events were observed in 3 patients $(2.5 \%)$, none of them required emergency surgery. In general, the diagnostic impact was $87 \%$.

Conclusions : VCE is a safe diagnostic method in pediatrics, starting of 3 years. VCE is an useful and non-invasive tool in the study of small bowel pathology.

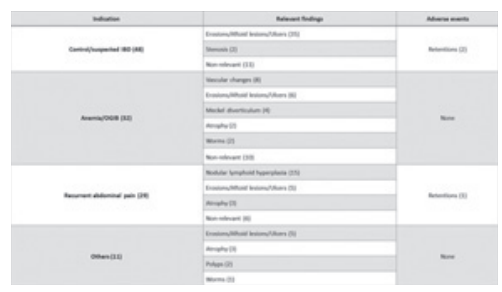

Videocapsule endoscopy

IP-32_G

Factors affecting the characteristics of advanced colorectal adenoma in diabetic patients

Kangwon National University Hospital

Ji Hyun Kim, Sung Joon Lee

Background : In recent years, the incidence of diabetes mellitus (DM) and colorectal cancer has been increasing in Korea. It is reported that hyperinsulinemia associated with type 2 DM leads to carcinogenesis through increased level of insulin-like growth factor 1. This study aimed to evaluate factors affecting the incidence of advanced colorectal adenomas known to be precancerous lesion, in diabetic patients of Korea.

Method: We retrospectively analyzed the data of patients with type $2 \mathrm{DM}$ who had colonoscopy between August 2008, and August 2016 in our hospital. Different variables were analyzed by multivariate logistic regression analysis to determine risk factors for development of advanced colorectal adenomas.

Results : 527 patients were included in the final analysis, and advanced adenomas were found in 65 patents $(12 \%)$. Patients in both groups were diagnosed with DM approximately 11 years prior to colonoscopy. Among 65 patients with advanced colorectal adenoma, 49 (77.8\%) were male. Lower HDL cholesterol (odds ratio 0.41, 95\% CI : 0.201-0.819) and higher LDL cholesterol (odds ratio 3.60, 95\% CI : 1.138-11.379) were significantly related to development of advanced colorectal adenomas ( $P$ value $0.01,0.03$ respectively).

Conclusion : LDL and HDL cholesterol were significantly related to advanced colorectal adenoma. There is no relationship between the level of $\mathrm{HbAlc}$ and advanved colorectal adenoma incidence. Further study will be needed to know the factors affecting the risk of advanced colorectal adenoma in diabetic mellitus.
IP-33_G

Adenoma detection with chromo endoscopy using oral indigo carmine

Nate Hospital ${ }^{1}$, Department of Gastrointestinal Medicine, Wakayama Medical University Hospital ${ }^{2)}$

OKoki Kawanishi' ${ }^{1}$, Takao Maekita ${ }^{2)}$, Yoshifumi Ikeda ${ }^{1)}$, Masahiko Furotani ${ }^{1)}$, Takayuki Kanno ${ }^{1)}$, Toru Niwa ${ }^{1)}$, Tsunehiro Nagaoka $^{1)}$, Keiichi Hatamaru ${ }^{2)}$, Takeichi Yoshida ${ }^{2}$, Yoshiyuki Ida ${ }^{2)}$, Mikitaka Iguchi' ${ }^{2)}$ Masayuki Kitano ${ }^{2)}$

Aim : Colonoscopy is currently the reference method to detect colorectal neoplasia, yet some adenomas remain undetected. Indigo carmine staining with various methods has shown interesting results for reducing this miss rate. The aim of this study was to determine if the standard colonoscopy prep followed by oral indigo carmine intake shows the increase in the adenoma detection rate (adenoma and adenocarcinoma ; ADR) and the mean number of adenomas per patient (MAP). Method : We introduced the oral indigo carmine technique to every subject and prospectively collected the data, and also performed retrospective review of endoscopic reports and medical charts of consecutive patients who underwent colonoscopy before introducing this technique to compare the results. Results : 211 subjects were included in the study (105 in indigo group). The ADR was not significantly different between the groups ; $44.8 \%$ in the indigo group versus $41.5 \%$ in the standard colonoscopy group (odds ratio, $1.15 ; 95 \%$ confidence interval, 0.9 $1.52, \mathrm{p}=0.33$ ). MAP was significantly greater in the indigo group (1.1) than in the standard colonoscopy group $(0.81 ; \mathrm{p}=0.008)$. For advanced adenomas, the results were $10(9.5 \%)$ and $7(6.6 \%)$, respectively $(\mathrm{p}=0.13)$. Conclusion : The routine use of oral indigo carmine does not lead to higher ADR regardless of higher MAP.

indigo carmine

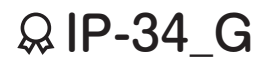

Polyp Recognition with You-Look-OnlyOnce (YOLO) Real-time Object Detection Systemin Colonoscopy

Department of Gastroenterology and Hepatology, Changi General Hospital ${ }^{1}$, GI Tech Ltd ${ }^{2}$

OJames Weiquan $\mathrm{Li}^{1 /}$, Tiing Leong Ang ${ }^{1)}$, Kenny De Wei Chong', Tiongsun $\mathrm{Chia}^{2}$ INTRODUCTION : Adenoma are missed in colonoscopy. Multiple
convoluted neural networks (CNNs) for polyp recognition are comconvoluted neural networks (CNNs) for polyp recognition are com-
putationally very expansive. You-Only-Look-Once version3 (YOLOv putationally very expansive. You-Only-Look-Once version3 (YOLOv
3 ) is a recently described, state-of-the-art, real-time object detection system using a single network evaluation for object classification and localization.

AIM : To evaluate the performance of YOLOv3 in detecting polyps from actual colonoscopy videos.

METHODS : Training data comprised 6,038 images from 18 "live" colonoscopy videos from a tertiary Singapore centre and live colonoscopy videos from a tertiary Singapore centre and
three public databases (CVC-300, CVC-ClinicDB and ETISthree public databases (CVC-300, CVC-ClinicDB and ETIS-
LaribPolypDB). Transferred learning was applied to improve feaLaribPolypDB). Transferred learning was applied to improve fea-
ture extraction using weights pre-trained with millions of images ture extraction using weights pre-trained with millions of images
from ImageNet. Validation performed using 2571 images. Testing from ImageNet. Validation performed using 2571 images. Testing
consisted 6,261 images of normal colon and sessile, pedunculated or flat-elevated polyps extracted from CVC-VideoClinicDB. Polyp masks in videos were converted to bounding boxes for consistency. Bounding boxes with intersection over union (IOU) area $>0.5$ are considered positive prediction.

RESULTS : Polyp detection rate was 100\% (all polyps in videos detected at least once). Our system reported a mean average precision (mAP) of 87.54 . Precision and recall $80.7 \%$ and $83.8 \%$, respecsion $(\mathrm{mAP})$ of 87.54 . Precision and recall $80.7 \%$ and $83.8 \%$, respec-
tively, which outperformed other algorithms tested on the same da-
tabase. F1 score 0.822 . Despite an ultra-deep CNN network with 106 tabase. F1 score 0.822 . Despite an ultra-deep CNN network with 106 achieved. Sensitivity and specificity per image $83.8 \%$ and $53.1 \%$ respectively.

CONCLUSION : YOLOv3 can classify and localise different polyps for real-time polyp detection using a single network evaluation. 


\section{IP-35_G}

Chemoprotective Activity of Skullcapflavone I against Aflatoxin-Induced Cytotoxicity and Genotoxicity : Mechanisms in THLE-3 Human Hepatocytes

De La Salle University ${ }^{11}$, National Research Institute of Chinese Medicine $^{2}$

OGlenn Oyong ${ }^{1)}$, Maria Carmen Tan ${ }^{1)}$, Chien-Chang Shen ${ }^{2)}$, Consolacion Ragasa ${ }^{1)}$

The protective effect of skullcapflavone I (SKI) against aflatoxin B1 (AFB1)-induced cytotoxicity and genotoxicity in THLE-3 including possible mechanisms were investigated. SKI previously elucidated and isolated from Andrographis paniculata were tested to be biocompatible with THLE-3 which were treated with or without SKI for $24 \mathrm{~h}$ before AFB1 exposure. Expressed Akt, Bcl2, and CYP $2 E 1$ transcripts were detected by qRT-PCR. Immunoblotting was performed for translated CYP2E1 including Akt and ERK1/2 phosphorylation. GST and AFB1 genotoxic metabolite, aflatoxin B1-8,9 epoxide (AFBO), were quantified. Comet assay was performed to assess DNA damage. AFB1 significantly downregulated the gene and protein expression of $\mathrm{Akt}, \mathrm{Bcl}-2$ while upregulating CYP2E1. Akt phosphorylation was inhibited while ERK1/2 activation increased. Increased comet tail length and moment connote DNA damage. SKI pre-treatment afforded cytoprotective effect in THLE3 viability with half-maximal effective dose of $8.46 \mu \mathrm{g} / \mathrm{mL}$. AFB1induced activities were reversed through dynamic Akt, Bcl-2 upregulation and CYP2E1 downregulation. Moreover, Akt activation increased while ERK1/2 phosphorylation was inhibited. GST significantly increased accompanied by decreased AFBO. Tail length and moment were comparable with untreated controls. Remarkably, LYS294002, a PI3K inhibitor, impaired SKI protective effect in THLE-3 suggesting key role of the pathway. This is the first report describing SKI protective activity against AFB1 toxicity in human hepatocytes and may help contribute to pharmaceutical leads targetting aflatoxin poisoning and carcinogenicity.

\section{IP-36_H 演題取り消し}

\section{Q}

Adherence to EASL (European Association for the Study of the Liver) guidelines in managing of spontaneous bacterial peritonitis (SBP).

Northwick Park Hospital, London North West University Healthcare NHS Trust ${ }^{1)}$, Ealing Hospital, London North West University Healthcare NHS Trust ${ }^{2}$

OMaria Bashyam ${ }^{1)}$, Vijay Manglam²), Sayyedd Adnan Aali' ${ }^{12}$,

Priya Khanna ${ }^{1)}$, Guduru Gopal Rao ${ }^{1)}$, Amit Amin ${ }^{11}$, Arabinda $\mathrm{Pal}^{2)}$, Jayantha Arnold ${ }^{2)}$, Meron Jacyna ${ }^{1)}$, David Sherman ${ }^{1)}$, AlRubaiy Laith ${ }^{1)}$, Sharif Amar ${ }^{11}$

Aim :

Diagnosis and prompt treatment of SBP is crucial as its associated mortality is upto $30 \%{ }^{1}$. We evaluated ascitic fluid (AF) analysis for SBP across our trust.

Method :

We did a retrospective analysis of AF from July-Dec2018 in cirrhotics, using online results systems. We evaluated SBP ( $>250$ - 2 entroprimary prophylaxis $(\mathrm{PP})$ when total protein $<15 \mathrm{~g} / \mathrm{L}$.

Results

Data showed 112 samples, 66 patients, median age61 and $71 \%$ with alcoholic cirrhosis. Reporting time was 2days average (4hours-10 days). $31 \%$ of requests mentioned SBP.

4 had SBP (average MELD17, CP-B9) no PP and reporting times of 4hours-7days. 2 had bacterascites and treated as significant. $66 \%$ had protein levels, $55 \%$ eligible for PP, 1 developed SBP (no PP), 14\% prescribed PP (nerage

Conclusion :

We observed delays in reporting AF and high mortality. Target areas are reporting times and considering PP. We are now implementing a trust ascites bundle, educating doctors and collaborating

1. EASL, EASL clinical practice guidelines on the management of ascites, spontaneous bacterial peritonitis, and hepatorenal syn-

SBP

Liver

IP-38_G

Effect of Hypoalbuminemia on the Mortality of Cirrhotic Patients with Spontaneous Bacterial Peritonitis

Division of Gastroenterology, Department of Medicine, Buddhist Dalin Tzu Chi Hospital ${ }^{1)}$, School of Medicine, Tzu Chi University ${ }^{2)}$ Tsung-Hsing Hung ${ }^{12}$

Background: The effects of hypoalbuminemia on the mortality of patients with cirrhosis and spontaneous bacterial peritonitis (SBP) have not been well determined.

Methods : We collected data from Taiwan's National Health Insurance Database on 14583 patients with cirrhosis and SBP who were hospitalized with SBP from 2010 to 2013. The 30-day and 3-year prognostic factors were calculated.

Results : Overall, 30-day and 3-year mortality were $21.9 \%$ and $77.7 \%$, respectively. After Cox proportional hazard regression model adjusted by the patients'gender, age, and comorbid disorders, old age (HR : 1.02, 95\% CI 1.01- 1.02, P<0.001), male (HR : $1.10,95 \%$ CI $1.02-1.20, \mathrm{P}=0.016$ ), esophageal variceal bleeding (HR : $1.23,95 \%$ CI 1.06- 1.43, $\mathrm{P}=0.006$ ), hepatocellular carcinoma (HR : $1.91,95 \%$ CI $1.77-2.05, \mathrm{P}<0.001$ ), hepatic encephalopathy (HR : $1.42,95 \%$ CI $1.30-1.55, \mathrm{P}<0.001$ ), hypoalbuminemia (HR : $1.62,95 \%$ CI 1.51- $1.74, \mathrm{P}<0.001$ ), renal function impairment (HR : $2.44,95 \%$ CI 2.25- 2.64, $\mathrm{P}<0.001$ ), non-alcoholic related cirrhosis (HR : 1.12, $95 \%$ CI 1.02- $1.24, \mathrm{P}=0.025)$, conferred higher risks for 30-day mortality in the cirrhotic patients with SBP. Patients with hypoalbuminemia also have poor 3-year survival (HR : $1.57,95 \%$ CI $1.50-1.63$, $\mathrm{P}<0.001)$.

Conclusion: In cirrhotic patients with SBP, the presence of hypoalbuminemia predicts poor short-term and long-term outcomes. 
Litholytic medication can be an alternative treatment in patients with gallbladder dyskinesia

Department of Internal Medicine, Gangnam Severance Hospital, Yonsei University College of Medicine ${ }^{1)}$, Department of Internal Medicine, Hallym University Dongtan Sacred Heart Hospital, Hallym University College of Medicine $^{2)}$, Department of Internal Medicine, Inha University School of Medicine ${ }^{3)}$, Department of Internal Medicine, Gachon University Gil Medical Center ${ }^{4)}$, Digestive Disease Center, CHA Bundang Medical Center, CHA University ${ }^{5)}$,

Department of Internal Medicine, Soonchunhyang University College of Medicine, Cheonan Hospital ${ }^{6)}$

OYoung Min $\mathrm{Kim}^{1)}$, Dong Hee $\mathrm{Koh}^{2)}$, Seok Jeong ${ }^{3)}$, Jae Hee

$\mathrm{Cho}^{4)}$, Chang-Il Kwon ${ }^{5)}$, Tae Hoon Lee ${ }^{6)}$, Dong Ki Lee ${ }^{1)}$, Sung Ill Jang $^{1)}$

\section{Background/Aims}

There was no consensus treatment guideline for gallbladder (GB) dyskinesia which diagnosed by reduced ejection fraction (EF) less than $38 \%$ measured with the ${ }^{99} \mathrm{Tc}^{\mathrm{m}}$-diisoproyl iminodiacetic acid effect of medical treatment on GB dyskinesia.

\section{Methods}

We retrospectively reviewed electronic medical records of enrolled 62 patients in 6 multicenter. The inclusion criteria of patients were as follows : (1) took litholytic drugs (ursodeoxycholic acid or a mixture of chenodeoxycholic acid and ursodeoxycholic acid), (2) under went DISIDA scan before and after medical treatment. We analyzed EF in all enrolled patients.

\section{Results}

EF of all enrolled patients was $34.4 \pm 22.6$ and it was significantly in creased by $53.8 \pm 26.8$ after medical treatment $(P<0.001)$. In 35 patients who diagnosed with GB dyskinesia, EF was $19.5 \pm 10.9$, and it was significantly improved by $47.9 \pm 27.3$ after medical treatment ( $P$ Conclusion

Litholytic drugs improved GB function. Especially, this medical treatment can be an alternative method on GB dyskinesia.
Gallbladder dyskinesia
litholytic drug 


\section{$\star$ Q IP-40_E}

Is Lumen-Apposing Metal Stents More Effective Than Plastic Stents for the Management of Pancreatic Fluid Collections : A Systematic Review and Meta-analysis

Affiliated Hospital of Southwest Medical University

OXiaowei Tang, Shali Tan, Yan Peng

Aim : We conducted this meta-analysis to investigate the role of lumen-apposing metal stents (LAMS) for pancreatic fluid collections (PFC).

Methods: We searched the studies from Pubmed, Medline, Embase and Cochrane databases from inception to November 2018. We extracted the data and analyzed the technical success, clinical success as well as the adverse events (AEs) of LAMS to evaluate its efficacy and safety. The pooled risk ratios (RR) is utilized to compare the clinical outcomes of LAMS with plastic stent.

Results : Eighteen studies including a total of 1391 patients are included. The pooled technical success of LAMS for PFC was 97\% [95\% confidence interval (CI): $95 \%-98 \%]$, and the pooled clinical success was $87 \%$ (95\% CI : 83\%-90\%). Regarding to AEs, the pooled event rate was $22 \% \quad$ (95\% CI : 16\%-29\%). Seven studies including 766 patients compared the clinical outcomes of LAMS with plastic stents. The pooled RR of technical success for LAMS and plastic stent was $1.01(95 \% \mathrm{CI}: 0.97-1.04, \mathrm{P}=0.72)$. For the clinical success, the pooled RR was $1.02(95 \% \mathrm{CI}: 0.98-1.07, \mathrm{P}=0.34)$. As for the the overall AEs, the pooled RR was 1.44 (95\% CI : 0.66-3.14, $\mathrm{P}=$ 0.36 ).

Conclusions : Our current study revealed that LAMS has no obvious advantages over plastic stents for PFC. Further randomized controlled trials comparing LAMS and plastic stents are warranted in future.

Lumen-Apposing Metal Stents (LAMS) pancreatic fluid collections (PFC)

\section{\&IP-41_G}

Angiopoietin-1 modified hair follicle mesenchymal stem cells (HF-MSCs) repair severe acute pancreatitis (SAP) via inducing angiogenesis in rats.

The Second Affiliated Hospital of Harbin Medical University OShizhu Jin

\section{BACKGROUND :}

Severe acute pancreatitis (SAP) is a high mortality disease in clinic. Previous research has demonstrated that stem cell have potentia for the treatment of SAP, Hair follicle-derived mesenchymal stem cells (HF-MSCs), with the advantages of abundant sources and convenient tiss.

\section{METHODS :}

In this research, to explore the therapeutic effects of HF-MSCs on severe acute pancreatitis, HF-MSCs were cultured and transplanted into pancreatis, and angiopoietin-1 modifid HF-MSCs (Ang-1-HF-MSCs) were generated MSCsectis MSCs co-localized with Ngn3, Pax4, PDX-1, and CD31, and the expression levels of these four makers of pancreatic stem cells were significantly increased after 2 weeks. Inflammatory response and angiogenesis were measured in pancreatic tissue samples, and the beneficial effects were evaluated through western blot immunohis-
tochemistry and immunofluorescence analysis.

\section{RESULTS :} Greater numbers of infused Ang-1-HF-MSCs were detected and co-
express Ngn3, Pax4, PDX-1 and CD31 in pancreas of rats with SAP than of control rats. It proved that HF-MSCs can differentiate into pancreatic cells and vascular endothelial cells. Infused Ang-1-HFMSCs promoted angiogenesis and reduced acinar cell necrosis in pression of anti-inflammatory mediators and reduced inflammation mediators in rats with SAP.

\section{CONCLUSIONS :}

Angiopoietin-1 modified HF-MSCs can repair injured pancreatic tissue via inducing angiogenesis in SAP rats.

IP-42_G

Effect of Calorie-Restriction on Severity of Experimental Acute Pancreatitis

All India Institute of Medical Sciences

OManish Kumar Sharma, Kumari Priyam, Tony George Jacob, Pramod Kumar Garg, Tara Sankar Roy

Impaired autophagy has been shown to be involved in pathogenesis of acute pancreatitis (AP). Calorie-restriction can induce autophagy. This can affect the outcome of experimental AP. Here, adult, male, Swiss albino mice weighing 20-25 grams were divided into three groups of six each. The first group (G1) was given equivalent doses of normal saline. The second group (G2) was not given any access to food during the night preceding the experimental induction by eight, hourly intraperitoneal injections of caerulein $(50 \mu \mathrm{g} / \mathrm{Kg}$ / dose). The third group (G3) was given free access to chow and water during the night and chow was removed just prior to inducing AP. All animals were sacrificed at the 9th hour after induction by $\mathrm{CO} 2$ narcosis. The pancreas was harvested. A part was either fixed in buffered paraformaldehyde or flash-frozen in liquid nitrogen. The histopathological score was estimated on H\&E-stained sections. Immunoblotting was used to study the expression of P62 (autophagic flux marker), LC3II/I (marker of induction of autophagy), HMGB1 (necrosis marker) and RIP1 (necroptosis marker). Animals of G3 had a higher histopathological score compared to G1 and G2 $(\mathrm{p}<0.001, \mathrm{p}<0.001)$. G3 also had lower expression of $\mathrm{P} 62$ and LC3II/I $(p=0.003)$ than G2. HMGB1 and RIP1 expression was significantly higher in G3 in comparison to G1 $(\mathrm{p}<0.001, \mathrm{p}<0.001$, respectively). Thus, calorie-restriction prior to induction of AP decreases the severity of AP.

IP-43_G Serum endocan as a novel biomarker for predicting survival in cirrhotic patients

Department of Hepatology, Kanazawa Medical University ONobuyuki Toshikuni, Mutsumi Tsuchishima, Mikihiro Tsutsumi

Aim : There are few biomarkers for predicting outcomes in cirrhotic patients. Endocan is a soluble proteoglycan that is secreted by activated vascular endothelial cells. The aim of this study was to examine the usefulness of serum endocan in predicting survival in cirrhotic patients. Methods : We enrolled 68 cirrhotic patients without a past history of hepatocellular carcinoma (median age 64 years, 36 men and 32 women ; $9 \mathrm{HBV}, 29 \mathrm{HCV}, 23$ alcoholic, and 7 others). Eight healthy people were used as a control. Results : (1) The median serum endocan levels were $1.1 \mathrm{ng} / \mathrm{ml}$ in healthy people and 1.9 $\mathrm{ng} / \mathrm{ml}$ in cirrhotic patients $(\mathrm{P}=0.013)$. Serum endocan levels increased with Child-Pugh grade progression (A/B/C, 1.6/2.6/3.1 ng/ $\mathrm{ml}, \mathrm{P}=0.018$ ). (2) During a median follow-up period of 10 years, 22 patients died. The cumulative survival rates at 5,10 , and 15 years were $97 \%, 87 \%$, and $73 \%$ in the patients with serum endocan levels $<2.0 \mathrm{ng} / \mathrm{mL}$ and $87 \%, 54 \%$ and $41 \%$ in those with levels $\geq 2.0 \mathrm{ng} /$ $\mathrm{mL}(\mathrm{P}=0.0049)$. A Cox proportional hazards regression analysis revealed that older age, Child-Pugh grades $\mathrm{B}$ and $\mathrm{C}$, definitive therapy failure, and elevated serum endocan levels $(\geq 2.0 \mathrm{ng} / \mathrm{ml}$; hazard ratio $1.6,95 \%$ confidence interval $1.0-2.8 ; \mathrm{P}=0.044)$ were predictors of poor survival. Conclusions : Serum endocan level reflects liver functional reserve and can be a survival predictor in cirrhotic patients. 
Outcomes of large volume paracentesis in cirrhotic patients with spontaneous bacterial peritonitis.

Aga Khan University Hospital

OHafiz Abdul Basit Siddiqui, Rabeea Azmat

Introduction :

Spontaneous bacterial peritonitis (SBP) is prevalent in decompensated cirrhosis with associated high mortality and incidence of acute kidney injury (AKI) and hepatorenal syndrome (HRS). Outcomes of LVP in SBP has not been clearly addressed. This study assessed the outcome of LVP in patients with SBP, in terms of mortality, length of stay and renal outcomes.

Methods :

We conducted a cross sectional study on 113 patients with diagnosed SBP. Among these patients 61 underwent LVP while 51 were managed conservatively. Baseline and 48 hours clinical outcomes were compared. Total length of stay, special care unit stay and need for RRT were assessed among other factors.

Results :

Statistically significant improvement seen in post 48 hours creatinine among patients undergoing LVP as shown in the table attached.

Conclusion :

LVP in SBP translates into significantly positive outcomes, Hence LVP is recommended as a favored therapeutic option.

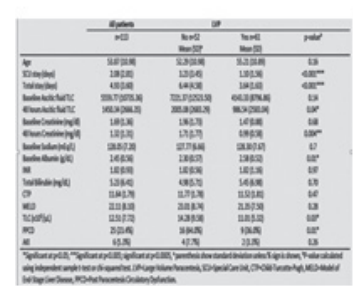

Large Volume Paracentesis Spontaneous Bacterial Peritonitis 


\section{IP-45_G}

Usefulness of Hepatobiliary Scintigraphy for Determining Cholecystectomy in Patients with Gallbladder Disease

Inje University Sanggye Paik Hospital

Ji Young Park, Tae Joo Jeon

The purpose of the study was to investigate the role of hepatobiliary scintigraphy in determining for cholecystectomy among patients with gallbladder disease. We retrospectively analyzed $713 \mathrm{pa}-$ tients who had 99mTc-diisopropyl-iminodiacetic acid (DISIDA) scan. The patients were classified as calculous cholecystitis (CC), acalculous cholecystitis (AC), symptomatic gallstones (SG) and asymptomatic gallstones (AG). We evaluated gallbladder motility with gallbladder ejection fraction (GBEF) estimated by DISIDA scan (Normal - GBEF $\geq 40 \%$, Abnormal - GBEF $<40 \%$ ), status of cholecystectomy and presence of recurrence after initial treatment. Patients with CC, AC, SG and AG were $267(37.4 \%), 135$ (18.9\%), $185(25.9 \%)$ and $126(17.7 \%)$, respectively. Patients who didn't get cholecystectomy despite of abnormal GB motility (group A) were 208 and the number of recurrence among them was 126 (60.6\%). Patients who didn't get cholecystectomy because they showed normal GB motility (group B) were 343 and the number of recurrence among them was $63(18.4 \%, \mathrm{p}<0.05)$. When we analyzed group A according to diagnosis, the number of patients were 102, 36, 51 and 19 and recurrent rates were $62(60.8 \%), 24(66.7 \%), 29(56.9 \%)$ and $11(57.9 \%)$, respectively. In group B, the number of patients were $73,72,93$ and 105 and recurrent rate were $29(39.7 \%), 8$ (11.1\%), 19 $(20.4 \%)$ and $7(6.7 \%)$, respectively $(\mathrm{p}<0.05)$.

DISIDA scan can be a useful indicator to determine cholecystectomy in patients with gallbladder disease. ing modified Look-Locker Inversion Recovery (MOLLI) sequence and pancreatic exocrine and endocrine function.

Department of Gastroenterology, Shinshu University School of Medicine $^{1)}$, Department of Radiology, Shinshu University School of Medicine $^{2)}$

ONorihiro Ashihara ${ }^{1)}$, Takayuki Watanabe ${ }^{1 /}$, Akira Yamada ${ }^{2)}$ Background :

Quantifying myocardial T1 values has been useful for detecting and characterizing fibrotic appearance in myocardial infarction, focal scars, and non-ischemic cardiomyopathies. Since pancreatic exocrine function decreases with chronic pancreatic fibrosis advancevalues and pancreatic exocrine and endocrine insufficiency.

Methods Thirty-two patients underwent abdominal contrast MRI in our de-
partment between October 2017 and February 2019. We evaluated T1 values with the MOLLI sequence, pancreatic exocrine insuffiE1) values, ar

Results :

Median cohort (9 male, 23 female) age was 71 (range : 49-84) years. Eighteen patients had pancreatic cysts, 3 had alcohol-induced chronic pancreatitis, 3 had pancreatic cancer, and 8 possessed other pancreatic features (autoimmune pancreatitis $: 2$, acute pancreatitis : 2 , bile duct tum
healthy control : 1).

A significant negative correlation was found between T1 and FE1 values $(\mathrm{r}=0.69, \mathrm{p}<0.01)$, with none between $\mathrm{T} 1$ and fasting insulin levels in the non-insulin administration group $(\mathrm{r}=0.044, \mathrm{p}=0.85)$. T 1 values were significantly higher in the insulin administration
than in the non-insulin group $(1655.5 \mathrm{vs} .898 .9 \mathrm{msec}, \mathrm{p}<0.01)$.

Conclusions : Pancreatic T1 values correlated with pancreatic exocrine function and might be useful in PEI diagnosis.
IP-47_G

Investigator-initiated prospective clinical study of the novel blood biomarkers for pancreatic cancer

Department of Gastroenterology, Kanazawa University Hospital OYoshio Sakai, Shuichi Kaneko

Pancreatic ductal adenocarcinoma (PDAC) is extremely difficult to be diagnosed in early stages. We previously reported that gene expression profile alteration of peripheral blood cells were observed with cancer development. We developed the blood mRNA screening system kit for pancreas cancer using gene expression profile alteration of PDAC patients'blood. The investigator-initiated clinical study to test the kit performance, which was designed in consultation with the Japanese PMDA, was performed.[Method] We selected significant 364 genes by analyzing PDAC and healthy subjects'blood mRNA expression data. We measured mRNA expression of the 364 genes and 17 house-keeping genes in 28 PDAC and 27 healthy subjects by real-time detection PCR, and established the equation for PDAC screening using the weighted linear sum of 56 genes'expression. After optimization of PCR method for these 56 genes, we developed the kit. The investigator-initiated clinical study to test the kit performance was performed in accordance with GCP.[Result] Sensitivity of the kit for 53 PDAC was $73.6 \%$. Specificities were $64.7 \%, 63.6 \%, 47.8 \%$ for 102 non-cancerous subjects, 23 IPMN, and 22 chronic pancreatitis patients. Among PDAC patients, 14 were in stage I, II, and 11 were positive with the kit. As for Pearson's correlation analysis between the kit and CEA or CA 19-9. $P$-values were $0.308,0.048$, and coefficients were 0.072 and 0.14 , respectively. [Conclusion] The study showed that the kit was possible alternative for PDAC screening.
IP-48_G

The quantitative monitoring of ct-DNA in patients with advanced PDAC undergoing chemotherapy

Department of Gastroenterology, Yokohama City University Makoto Sugimori, Kazuya Sugimori, Shin Maeda

Aim : The aim of this study was to evaluate the utility of quantitative monitoring ct-DNA using digital PCR (dPCR) in patients with advanced PDAC undergoing chemotherapy.

Method : The tissue-DNA and ct-DNA were obtained from 35 cases with advanced PDAC, and KRAS mutation was detected using dPCR. From 18 cases, serum samples were obtained every 4-8 weeks during chemotherapy, and the quantitative monitoring of KRAS mutation using dPCR was also performed.

Results : In the tissue-DNA analysis, 34/35 cases were KRAS mutation positive. And NGS revealed KRAS mutation negative case harbored NRAS Q61R. In the ct-DNA analysis, KRAS mutation was detected in 17/35 cases (liver or lung metastasis : 13/14 (mutation allele frequency (MAF) : $0.1 \%-31.7 \%$ ), peritoneal metastasis : $2 / 9(0.1 \%)$, locally advanced $: 2 / 12(0.1 \%))$. In the quantitative monitoring, the MAF value changed in concordance with the disease state. In 3 locally advanced cases, KRAS mutation was started to detect at the same or earlier time of liver metastasis. In 5 liver metastasis cases, KRAS mutation was disappeared during SD or $\mathrm{PR}$, and was reappeared at the same or earlier time of PD. The median PFS was significantly longer in KRAS mutation disappeared cases than in continuously detected cases (216 versus 51 days, $p=$ $0.0007)$.

Conclusion : The quantitative monitoring of ct-DNA using dPCR is valuable for monitoring disease state. The disappearance of KRAS mutation may be useful as a prognostic factor. 
IP-49G Identification of a druggable target in pancreatic cancer with specific genetic defects

National Health Research Institutes

OWen-Chun Hung, Tzu-Lei Kuo, Li-Tzong Chen

K-ras mutation and p53 loss are frequently found in pancreatic cancer. In addition, the WNT/B-catenin signaling molecules are also highly mutated. However, the genetic interaction between K-ras, $\mathrm{p}$ 53 and WNT/B-catenin in pancreatic tumorigenesis is still unclear. We generated Pdx1-CreKrasG12Dp53L/+APCL/+ (KPA) and Pdx 1-CreKrasG12Dp53L/+ (KPC) mice and compared their phenotypes in vivo. Our results demonstrated that survival of the KPA mice was shorter than that of the KPC mice. We isolated cancer cells from mouse tumors and found that the KPA cancer cells are highly invasive and exhibit distorted morphology in organoid culture with extensive invadopodia formation and elevated matrix metalloproteinase (MMP) activity. Moreover, the platelet-derived growth factor (PDGF) pathway is upregulated in the KPA cancer cells, and PDGF production induced by $B$-catenin triggers constitutive activation of the Src kinase via the PDGF receptor in the cells. Treatment of KPA mice with Src inhibitor significantly suppressed tumor growth in mice. In clinical setting, patient's serum PDGF level is correlated with the expression of PDGF and phosphor-Src in tumors and elevated PDGF/phosphor-Src level in tumors predicts increased recurrence and poor survival. Mutations of the WNT/Bcatenin signaling molecules are also higher in patients with elevated PDGF/phosphor-Src level. Collectively, we identify Src as a druggable target in pancreatic cancer with K-ras, p53 and WNT/Bcatenin defects. 


\section{Q}

Quality Improvement Project for DNA (Did-Not-Attend) Rates at our hospital's Endoscopy Unit during September 2018 March 2019

London Northwest Healthcare, Central Middlesex Hospital ${ }^{1)}$, London Northwest Healthcare, St Mark's Hospital ${ }^{2}$

OKinjal Patel ${ }^{1)}$, Maria Bashyam ${ }^{1.2)}$, John Walton ${ }^{1.2)}$, Ian Johnston $^{1.2)}$

Introduction :

Nonattendance at outpatient endoscopy appointments has financial
and productivity implications on the NHS. Common causes for DNAs include forgetfulness and communication failures. However.
tal factors play a role.

The Endoscopy Unit at our hospital offers services for our hospital
and Northwick Park Hospital with 2 affiliated units at our hospital. Last year, our hospital's DNA's were $6 \%$, above the standard $<5 \%$.

Aim

Analysis of the DNA rates at our hospital's endoscopy unit over the
last 6 months. Implement change and remeasure the outcome. Method

Retrospective audit of the DNA's/No shows from the $05 / 09 / 18$ to
$05 / 03 / 19$, using Endosoft software and hard copies of procedure

Results :

In this time period, 4027 appointments were made, $7 \%$ were DNA's. physicians with the highest DNA rates were Gastroenterologist

Demographically, $58 \%$ of DNAs were male, $61 \%$ aged $>50$ and $59 \%$ from outside Brent

Conclusion

Non-urgent referrals from Gastroenterologist, GPs and Surgeons are likelier DNA's. Those living further away from our hospital are We recommend the following

Pre-assessment for routine OGDs.

Sending Multiple reminder SMS, supplemented with calls if needed.

Ascertain reasons for DNAs.

Re-measure the DNA rate September 2019 gastrointestinal bleeding : a systematic review and meta-analysis

Affiliated Hospital of Southwest Medical University

OXiaowei Tang, Chunyu Zhong, Yan Peng

Aim : Recent studies have shown that over-the-scope-clip (OTSC) system allowed for effective hemostasis for refractory GI bleeding lesions. So we aimed to conduct a systematic review to evaluate the effectiveness and safety of the OTSC system for management of acute non-variceal upper GI bleeding.

Method: A comprehensive literature search was conducted on PubMed, EMBASE, and Cochrane Library covering the period from January 2007 to December 2018. The search terms included "gastrointestinal bleeding" AND "OTSC OR over the scope clip". The literature was selected independently by two reviewers according to the inclusion and exclusion criteria. The statistical analysis was carried out using Comprehensive Meta-Analysis software version 3.0.

Results : A total of 15 studies including 669 patients with 678 GI bleeding lesions were identified. The major cause for GI bleeding was the peptic ulcer $(70.61 \%)$. Pooled technical success was achieved in 662 lesions $(95.4 \% ; 95 \%$ confidence interval (CI), 93.0\%-97.0\%), and the pooled clinical success was achieved in 591 lesions $(85.1 \% ; 95 \%$ CI $77.9 \%-90.2 \%)$. The incidence of re-bleeding was reported in 64 patients and the post-procedure 30-day mortality was $10.2 \%(n=68)$. Only $2(0.3 \%)$ patients occurred complications after OTSC system procedure.

Conclusions : Our study demonstrated that the OTSC system was a technically feasible modality and highly efficacious in achieving hemostasis in acute non-variceal upper gastrointestinal bleeding.

\section{IP-52 E EUS-guided angiotherapy of bleeding GIST}

University Hospital Virgen Macarena

$\bigcirc$ Rafael Romero-Castro, Victoria Alejandra Jimenez-Garcia, Pedro Hergueta-Delgado, Antonio Garrido-Serrano, Reyes Aparcero-Lopez, Sofia Pereira-Gallardo, Angel Caunedo-Alvarez

Management of bleeding GIST is challenging. Several endoscopic procedures as a bridge therapy until surgical therapy such as cyanoacrylate (CYA) or polidocanol injection, clipping, detachable snare loop, band-ligation and hemostatic powders have been reported.

We report three cases of bleeding GIST successfully treated under EUS-guidance. Patients characteristics, type of procedure and clinical outcome are shown in the table. One adverse event was observed. A bleeding into the tumor cavity after the punction of a $22 \mathrm{G}$ needle, succesfully managed by EUS-guided deployement of a coil plus CYA. Clinical outcome in the three patients was uneventful until scheduled surgery. Histopathology confirmed there all were GIST.

EUS-guided therapy of bleeding GIST by injecting CYA, deploying coils or a combination of both was effective in these three cases. EUS-guided angioterapy could be another endoscopic procedure in bleeding GIST aiming to obtained hemostasis until scheduled surgical therapy.

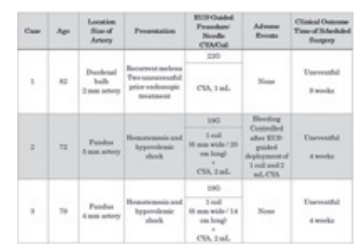

EUS-guided angiotherapy

GIST

|P-53_E Diagnostic utility of Endoscopic ultrasonography-elastography in gastric submucosal tumors : A pilot stud

\section{CHA Bundang Medical Center} OJong Woo Kim

Background : The etiology of gastric submucosal tumors (SMT) is assessed by endoscopic ultrasound (EUS) and EUS guided fine needle aspiration (FNA). However, despite of the invasive procedures, it is often difficult to obtain specimens. Therefore we investigated the potential of endoscopic ultrasound elastography (EUS-EG) using strain ratio to differentiate among gastric SMTs

Methods: We prospectively registered 10 patients with gastric SMT diagnosed by esophagogastroduodenoscopy in our medical center between April 2018 and August 2018. The patients underwent EUS-EG (EG-3670URK ; PENTAX Medical) combined with diagnostic ultrasound imaging systems (ARIETTA 70a ; Hitachi Aloka Medical LTd) and were assessed the strain ratio. Pathologic confirmations were done.

Results : Of the 10 patients with a confirmed diagnosis, eight had leiomyoma, one had schwannoma, and one had hamartoma. The most common lesion site was the cardia and the esophagus in three patients $(30 \%)$, respectively, followed by the body in two patients $(20 \%)$, and the antrum and the duodenum in one $(10 \%)$ each. Tumor diameter was $3-28 \mathrm{~mm}$. With regard to the strain ratios, leiomyomas showed relatively low value ranging from 2.0 to 9.2 . On the other hand, Schwannoma and duodenal hamartoma showed higher strain ratio, 62.0 and 26.4 , respectively.

Conclusions : EUS- elastography could be a promising diagnostic tool for defining the characteristics of gastric SMT. However, further studies with large sample size are necessary to elucidate the efficacy of elastography in differential diagnosis of gastric SMTs. 


\section{IP-54_E}

Endoscopic management of iatrogenic gastrointestinal defects with the Over-the-Scope Clip (OTSC) System : an updated systematic review

Affiliated Hospital of Southwest Medical University OXiaowei Tang, Chunyu Zhong, Yan Peng

Aim : Over-the-scope-clip system (OTSC) have been reported to be effective for gastrointestinal (GI) defects. So we aimed to conducted an updated systematic review to evaluate the clinical safety and efficacy of the OTSC system for management of iatrogenic GI defects.

Methods : Studies published in PubMed, Embase and Cochrane library from January 2006 to June 2018 were searched using the following terms, "defect OR perforation" AND "OTSC OR over the scope clip". The literature was selected independently by two reviewers according to inclusion and exclusion criteria. The statistical analysis was carried out using Comprehensive Meta Analysis software version 3.0.

Results : A total of 12 studies including 191 patients with iatrogenic GI defects were identified. All the studies were published in the Europe $(n=7)$ and the United States $(n=5)$. The major causes for iatrogenic GI defects were endoscopic submucosal dissection ( $\mathrm{n}=$ $79)$ and endoscopic mucosal resection $(\mathrm{n}=31)$. Pooled technical success was achieved in 182 patients $(89.1 \% ; 95 \%$ confidence interval (CI), $81.6 \%-93.8 \%$ ), and the pooled clinical success was achieved in 170 patients $(85.2 \% ; 95 \%$ CI, $71.9 \%-92.8 \%) .2$ patients ( $1 \%)$ occurred complications after OTSC system procedure.

Conclusion : Our study revealed that endoscopic closure of iatrogenic GI defects by OTSC system was a safe and effective approach. Further randomized controlled trials with large sample are warranted to compare OTSC system to others treatment modalities in future.
Clinical outcomes of lumen-apposing metal stent in the management of benign gastrointestinal strictures : a systematic review and metaanalysis

Affiliated Hospital of Southwest Medical University OXiaowei Tang, Shali Tan, Yan Peng

Aim : The aim of this meta-analysis was to determine the efficacy and safety of lumen-apposing metal stent (LAMS) for benign gastrointestinal (GI) strictures.

Methods: Medline, Embase, Cochrane and PubMed databases were searched using the keywords "benign stricture", "gastrointestinal stricture","stricture","lumen-apposing metal stent", "metal stent", "AXIOS", "self-expandable stents" on December 2018. Articles were selected for review by two authors independently on the basis of predefined inclusion criteria and exclusion criteria. A metaanalysis using a random effects model was performed.

Results : Six studies with a total of 144 patients were included in the final analysis (60 males, $41.7 \%$ ). Overall, the pooled technical success rate was $98.3 \%$ [95\% confidence interval (CI) : 0.962-1.004], clinical success rate was $73.8 \%(95 \%$ CI : $0.563-0.912)$ and adverse events rate was $30.6 \%$ (95\% CI : $0.187-0.425)$. The most common complication associated with LAMS for benign GI strictures was migration, and the pooled events rate was $10.9 \%$ (95\% CI : 0.058 0.160 ). According to locations of stricture, subgroup analysis was performed in terms of clinical success [Esophagogastric : $63.9 \%$ (95\% CI : 0.365-0.914) ; Gastroduodenal : $67.4 \%$ (95\% CI : $0.421-$ 0.927) ; Gastrojejunal : 78\% (95\% CI : 0.638-0.922) ; Pylorus : $77.6 \% \quad(95 \%$ CI $: 0.551-1.002)$; Colonic : $85.3 \%$ (95\% CI : $0.515-$ 1.191)].

Conclusions : LAMS can results in most patients obtaining clinical symptom improvement or resolution, and it might be an alluring prospect for treating patients with this difficult condition.

\section{Q IP-56_E}

Clinicopathologic and endoscopic features of Helicobacter pylori negative early gastric cancer treated by endoscopic submucosal dissection

Department of Internal Medicine, Kosin University College of Medicine

OKyoungwon Jung, Jangho Park, Jae Hyun Kim, Sung Eun

Kim, Won Moon, Moo In Park, Seun Ja Park

The aim of this study was to evaluate features of Helicobacter pylori negative gastric cancer ( $\mathrm{HpNGC)}$ treated by endoscopic resection (ER).

Patients with early gastric cancer (EGC) who had received all three tests of Helicobacter pylori (Hp) were included. The definition of HpNGC was limited to negative cases in which there was no previous history of eradication and all negative results of serologic test, rapid urease test and polymerase chain reaction of gastric specimen. Clinical, endoscopic, and pathologic findings analyzed retrospectively

A total of 89 patients (61.8\%) had ER in 144 patients diagnosed EGC. In EGC patients who underwent ER, 21 patients were negative for all three tests related to Helicobacter. According to the Hp positivity, there were no significant differences between two groups except female predominance of HpNGC. Both male and intestinal metaplasia were identified as risk factors for recurrence in both univariate $(\mathrm{p}=0.026$ and $\mathrm{p}=0.033)$ and multivariate analyzes (Odds ratio (OR) 10.501, $\mathrm{p}=0.033$ and $\mathrm{OR} 5.65, \mathrm{p}=0.040)$. In patients with HpNGC, endoscopic findings showed atrophy in all patients, intestinal metaplasia in $66.7 \%$.

Considering clinical features and background of the endoscopic mucosa, atrophic change and intestinal metaplasia may be more closely related to carcinogenesis than the positivity of $\mathrm{Hp}$. Further studies are needed to clarify the importance of mucosal change.

Early gastric cancer

Helicobacter pylori negative gastric cancer
The impact of mentoring by Japanese expert endoscopists on the learning curve of a Western trainee in endoscopic submucosal dissection at a European tertiary referral center

Department of Internal Medicine III, Medical University of Vienna ${ }^{1)}$, Department of Gastroenterology, Nippon Medical School ${ }^{2)}$, IMed19 $9^{3)}$

OWerner Dolak ${ }^{1}$, Osamu Goto ${ }^{2)}$, Yumiko Kamogawa-Schifter ${ }^{1)}$ Wolfgang Sieghart ${ }^{3)}$, Maximilian Schoeniger-Hekele ${ }^{1)}$, Barbara Tribl $^{1}$, Michael Trauner ${ }^{1}$

INTRODUCTION : Improving skills in endoscopic submucosal dissection (ESD) is difficult in Europe. Mentoring by Japanese expert endoscopists may help to overcome this issue.

METHODS : After structured learning at a large Asian referral center for ESD Western ESD trainee (WD) performed some own ESD cases before doing an observership in Japan. Hereafter, he received lesion specific mentoring for his next ESD cases (provided by e-mail). This is an analysis of his ESD learning curve.

RESULTS : Within 18 months Western ESD trainee performed ESD in 22 consecutive patients (male, $\mathrm{n}=17$; median age 63 years, range 37-81 years) : rectum, $n=8$; esophagus, $n=4$; colon, $n=4$; stomach, $\mathrm{n}=3$; esophagogastric junction, $\mathrm{n}=3 ; 17 / 22$ cases $(77 \%)$ were malignant. Mean specimen size was $35 \pm 17 \mathrm{~mm}$. Mentoring was applied to the 14 most recent cases, resulting in better en-bloc resection rate $(12 / 14,86 \%$ vs. $5 / 8,62 \%, p=0.032$, Fisher's exact test) and reduction of serious adverse events (3 perforations, all within the initial 8 cases).

CONCLUSION : Although the number observed is low, mentoring by Japanese expert endoscopists seems to be beneficial for the learning curve of a Western ESD trainee, resulting in better en-bloc resection rate and less serious adverse events. 


\section{IP-58 E}

Endoscopic submucosal dissection for colorectal non-invasive neoplasms : a single medical center report

Division of Hepato-Gastroenterology, Department of Internal Medicine, Kaohsiung Chang Gung Memorial Hospital

Wei-Chen Tai, Yu-Chi Lee, Long-Sheng Lu

\section{Introduction}

Endoscopic submucosal dissection (ESD) is an effective therapeutic endoscopic procedure for treating noninvasive colorectal tumors. The en bloc resection rate of ESD is high than conventional EMR (endoscopic mucosa resection). We aimed to evaluate the safety and clinical efficacy of colorectal ESD in Kaohsiung CGMH.

\section{Patient and Methods}

We retrospectively enrolled the patients who received colon rectal ESD between January 2012 and April 2018 from our hospital. We record the information by chart review, including the patient characteristics, indication for ESD, the size and pattern of tumor, the procedure times, the post ESD complication rates, the en bloc resection rate, and pathologic features of tumors.

\section{Result}

115 patients (70 male, 61\%) who underwent colorectal ESD were enrolled into this study. The mean age is 64.6 years old. The colorectal tumor location are located cecum $(24.3 \%)$, A-colon $(20 \%)$. Ttal tumor location are located cecum (24.3\%), A-colon (20\%), T-
colon (13.9\%), D-colon (5\%), S-colon (12.1\%), Rectum (17.3\%). colon $(13.9 \%)$, D-colon $(5 \%)$, S-colon $(12.1 \%)$, Rectum $(17.3 \%)$.
The average ESD time is 67.2 minutes. The rates of immediate and The average ESD time is 67.2 minutes. The rates of immediate and
delayed major bleeding rate were $3.4 \%$, respectively, while there were no patients with immediate or delayed perforation in this study. After an average postoperative follow up of 21.1 months, the rate of tumor recurrence was $0 \%$

\section{Conclusion}

Our data of colorectal ESD showed that colorectal ESD is a safe and Our data of colorectal ESD showed that colorectal ESD is a safe and
effective procedure for colorectal non-invasive tumors and wareffective procedure for colorectal non-in
rants consideration as first-line therapy.

Colon neoplasm

IP-59_E

Modified Pulley Traction System in Endoscopic Submucosal Dissection (ESD) of Colonic Lesions Department of Gastroenterology \& Hepatology, Tan Tock Seng Hospital $^{1}$, Department of Gastrointestinal Oncology, Osaka International Cancer Institute ${ }^{2)}$

OStephen Tsao ${ }^{1)}$, Mann Yie Thian" ${ }^{1)}$ Yoji Takeuchi' ${ }^{2)}$, Noriya $\mathrm{Uedo}^{2)}$

\section{Background :}

ESD is a proven technique for endoscopic treatment of early gastrointeestinal tract neoplasia. Technical challenges remain especially when resecting large lesions in difficult locations. Traction system providing unilateral traction has been described as a useful tool. We present a case series using modified pulley traction system in assisting colonic ESD.

\section{Methods :} Dental floss was used as the traction line. It was inserted through
the biopsy channel and a loop was tied outside the endoscope bethe biopsy channel and a loop was tied outside the endoscope be-
fore procedure. Circumferential incision with Flush Knife BT 1.5 $\mathrm{mm}$ Fujifilm was carried out at the targeted lesion after submucosal lifting. The dental floss loop outside the endoscope was cut and tied to an endoclip which was then placed over the lesion's distal margin. Another endoclip was placed over the existing line on the opposite wall distally creating a pulley traction system.

\section{Results :}

Three colonic ESD cases were carried out. Site of lesions were rectum $(3.7 \times 3.0 \mathrm{~cm})$, ascending colon $(4.0 \times 3.5 \mathrm{~cm})$ and distal sigmoid colon $(1.8 \times 1.5 \mathrm{~cm})$. Average completion time was $169.3 \mathrm{minutes}$. No perforation occured. Enbloc resection was achieved in all cases.br $>$ Conclusion :

The modified pulley traction system was helpful in achieving en bloc resection especially at difficult locations. This method provides an alternative where similar accesories are limited outside Japan, where as dental floss and endoclips are readily available throughout the world.

\section{IP-60 E Outcomes OF PATIENTS With DE- LAYED SURVEILLANCE COLONOSCOPIES}

Bay of Plenty District Health Board, Tauranga Hospital OCameron Schauer, Allan Plant, Alain Vandal, Adrian Claydon

\section{Background/Method :}

Surveillance colonoscopy has been shown to be an effective tool for prevention of colorectal cancer (CRC) in high-risk populations, if ad hered to. We aimed to discover the sequelae of late surveillance in a cohort of patients overdue for colonoscopy, in particular the development of CRC or advanced adenoma (AA) via comparison of overdue procedures (later than 90 days) to those whose surveillance was completed either early or on time over a 12 year period. The primary outcome measured was the incidence of adenocarcinoma and mary

\section{Results :}

786 patients were recorded as overdue for surveillance colonoscopy, and 386 were completed early or on time. The mean time overdue was 26.0 months. 4 cases $(0.50 \%)$ of CRC were found in patients overdue compared to 6 cases $(1.5 \%)$ for those done on time $(\mathrm{p}=0.13)$. There were $86(11.0 \%)$ AA in patients overdue compared to $27(7.0 \%)$ in those not overdue $(\mathrm{p}=0.022)$. Overdue status increased the expected risk of AA by approximately $64 \%$ (95\% CI $[4 \%, 158 \%], \mathrm{p}=0.03)$ or $18 \%$ per year overdue $(95 \%$ CI $[5 \%, 29 \%]$, $\mathrm{p}=0.004)$. The adjusted risk associated with previous polyps as the indication for surveillance is considerable at 78\% (95\% CI [15\%, $176 \%], \mathrm{p}=0.009)$. Absolute polyp count increases in expectation by 0.12 for each year overdue $(95 \%$ CI $[0.05,0.19], \mathrm{p}=0.001)$.

\section{Conclusion :}

Overdue surveillance is not predictive of increased CRC but does increase expected number of AA. malignant colorectal large bowel obstruction following endoscopic stenting: A comparison of oncologic outcomes.

University Surgical Cluster, National University Health ${ }^{1)}$, Yong Loo Lin School of Medicine, National University of Singapore ${ }^{2)}$ $\bigcirc$ Heidi SY Chang ${ }^{1)}$, Tian Zhi Lim ${ }^{11}$, Pei Yin Sim²), Dedrick Chan $^{1)}$, Ker-Kan Tan ${ }^{1.2)}$

\section{Purpose}

To evaluate oncological outcomes between laparoscopic and open resection of colorectal cancer presenting with large bowel obstruction following endoscopic stenting.

\section{Methodology}

A retrospective review of all patients with left-sided obstructed colorectal cancer who underwent endoscopic stenting and elective orectal cancer who under
surgery for curative intent.

\section{Results}

Between January 2007 and June 2016, 39 patients were included. 23 underwent laparoscopic resection, the rest underwent open surgery. Both groups were comparable in co-morbidities, cancer stage and site of obstruction. The sigmoid colon (53.8\%) was the most common site of obstruction. There was no difference in grades of complication. Median length of hospital stay ( $p: 0.018)$ was shorter
in the laparoscopic group. Median follow-up was 64 months $(9.5-132)$ with no difference in
overall survival $(p: 0.784)$. Overall recurrence was $14(36 \%) .8$ paoverall survival $(p: 0.784)$. Overall recurrence was $14(36 \%)$. 8 pa-
tients had local recurrence divided between anastomotic site and peritoneal disease equally. Recurrence rates did not increase when surgery was more than 3-weeks from stent insertion. Laparoscopic resection was observed to have a higher recurrence rate (11 vs 3$)$
$(p: 0.098)$ despite comparable intra-operative lymph nodes harvested and uptake for adjuvant chemotherapy.

\section{Conclusion}

Laparoscopic resection of colorectal cancer presenting with obparable long-term oncological outcomes.

Colorectal Cancer Endoscopic Stenting 


\section{Q}

Ping Tung Christian Hospital

OYoen Young Chuah, Lian Feng Lin, Yi Chun Chan, Seng Howe Nguang

\section{Background/Aims :}

Upper gastrointestinal endoscopic surveillance is usually the first approach in suspected patients of GERD in Taiwan. Yet, there have been a few studies looking into the association of gastroesophageal flap valve function (GEFV) to endoscopic grading of GERD and gastrointestinal symptoms in Taiwanese patients. The primary aim of trointestinal symptoms in Taiwanese patients. The primary aim of
this study was to investigate (1) the association between GEFV (Hill classification) and endoscopic grading of GERD (Los Angeles Classification), and (2) the correlation of GEFV with upper gastrointestinal symptoms in Taiwanese patients.

\section{Patients and methods :}

Patients were included into the study and the GEFV graded I to IV Patients were included into the study and the GEFV graded I to IV as follows : Hill I-II : normal GEFV, and Hill III-IV : abnormal GEF V. GERD L.A. classification was as follows : L.A. A-B :
flux esophagitis and L.A. C-D : severe reflux esophagitis.

\section{RESULTS :}

225 patients were recruited. Of these patients, normal GEFV group and abnormal GEFV group consisted of $86.2 \% \quad(n=194)$ and $13.8 \%$ $(\mathrm{n}=31)$ respectively. Patients in abnormal GEFV group had a severe grading of GERD ( $16.1 \%$ vs $2.1 \% \mathrm{p}=0.03)$. Abnormal GEFV group had higher chance of having foreign sensation at throat $(9.7 \%$ (16.1\% sensation at throat $(9.7 \%$ (1\%, $=0.02$ ) if compared to normal GEFV group.

\section{CONCLUSIONS :}

Patients with abnormal valves (Hill grades III and IV) are associated with severe grading of GERD in endoscopy. Abnormal GEFV is more correlated to gastrointestinal symptom of foreign sensation at throat.

\section{IP-64_E}

Characteristics of achalasia patients associated with developing esophageal squamous cell carcinoma

Digestive Diseases Center, Showa University Koto Toyosu

Hospital $^{1 /}$, Digestive Diseases Center, Showa University Northern Yokohama Hospital ${ }^{2}$

$\bigcirc$ Mary Raina Angeli Abad $^{11}$, Yugo Iwaya ${ }^{1)}$, Yohei Nishikawa ${ }^{12}$, Kosuke Kunita $^{1)}$, Akiko Ueno ${ }^{1)}$, Yuto Shimamura ${ }^{1)}$, Mayo Tanabe $^{1)}$, Kazuya Sumi ${ }^{1)}$, Hideomi Tomida ${ }^{1)}$, Haruo Ikeda ${ }^{1)}$, Manabu Onimaru ${ }^{1)}$, Shin-ei Kudo ${ }^{2)}$, Haruhiro Inoue ${ }^{1}$

Background and Aim : Achalasia has been considered as one of the risk factors for developing SCC. This study aims to evaluate the characteristics of achalasia patients associated with developing SCC.

Methods : This is a retrospective analysis study performed between January 2010 and March 2018. We reviewed the database of patients diagnosed with esophageal achalasia based on Chicago Classification.

Results : From a total of 1253 achalasia patients, 16 patients $(1.28 \%)$ were identified to have SCC. Ten $(62.5 \%)$ were males and $6(37.5 \%)$ were females. Median age was $68(\mathrm{IQR}=56.25-76)$. Based on Chicago Classification, the type of achalasia seen were as follows : Type I/II/III $=8 / 6 / 2$. On histopathology results, 15 patients $(93.75 \%)$ had mucosal SCC, and 1 patient $(6.25 \%)$ had SCC extending to the submucosal layer. Achalasia patients with SCC were noted to be more likely in the older population $(p=<0.001)$ and with a higher prevalence of sigmoid type $(\mathrm{p}=<0.001)$ compared to achalasia patients without SCC. All patients underwent successful endoscopic resection (ER) without any complications.

Conclusion : Achalasia patients in the older population with a sigmoid type are at a higher risk for developing SCC and should be monitored more closely during follow-up.

achalasia

squamous cell carcinoma

\section{요 IP-65_E}

ENDOSCOPIC SUBMUCOSAL DISSECTION VS ENDOSCOPIC MUCOSAL RESECTION FOR BARRETT'S SUPERFICIAL ESOPHAGEAL NEOPLASIA : WESTERN MULTICENTER STUDY

Department of Internal Medicine, Cleveland Clinic ${ }^{1}$, Department of Gastroenterology, University of Florida ${ }^{2}$, Department of Gastroenterology, University Hospitals ${ }^{3}$, National Cancer Center Hospi$\mathrm{tal}^{4)}$, Department of Gastroenterology, Cleveland Clinic ${ }^{5)}$ $\bigcirc$ Lady Katherine Mejia Perez $z^{1)}$ Omar Alaber ${ }^{3)}$, Salmaan Jawaid $^{2)}$, Peter Draganov ${ }^{2)}$, Amitabh $\mathrm{Chak}^{3)}$, Yutaka Saito ${ }^{4)}$, Seiichiro Abe ${ }^{4)}$, Amit Bhatt ${ }^{5)}$

Background : Clinical outcomes of endoscopic resection for Barrett's superficial neoplasia remain unclear.

Aim : To compare recurrence rates of endoscopic mucosal resection and endoscopic submucosal dissection for Barrett's superficial neoplasia.

Methods : Multicenter study in the U.S. including patients who underwent ESD or EMR for BE-HGD/TlaEAC. Primary outcome was composite of metastasis and local recurrence rate.

Results : 98 patients underwent EMR and 49 ESD. ESD had higher en bloc, R0, and curative resection rates than EMR. The 12-month local recurrence rate for EMR and ESD was $63 \%$ and $11 \%$ ( $p$ $<.0001)$.

Conclusions : ESD results in more definitive treatment of BE-associated early neoplasia than EMR, with lower recurrence rates and less need for repeat therapeutic endoscopic procedures.

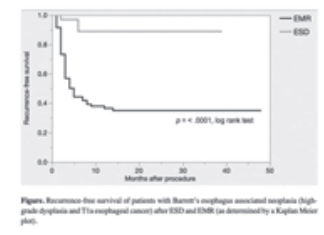




\section{IP-66_E}

ENDOSCOPIC REMOVAL OF FOREIGN BODIES : A RETROSPECTIVE STUDY IN A REFERRAL CENTER IN JAPAN

Division of Gastroenterology and Hepatology, Department of Internal Medicine, Keio University School of Medicine ${ }^{1)}$, Center for Diagnostic and Therapeutic Endoscopy, Keio University School of Medicine ${ }^{2)}$

OKenji Jose Luis Limpias Kamiya ${ }^{1)}$, Naoki Hosoe ${ }^{2)}$, Haruhiko

Ogata $^{2)}$, Takanori Kanai ${ }^{1)}$

Ingestion of foreign bodies (FB) and food bolus impaction (FBI) in the digestive tract is a common clinical problem, however until now there are no previous reports of endoscopic management of $\mathrm{FB}$ in Japan. The aim of this study is to report the level of safety and efficacy in the management of FB and FBI in our endoscopy center.

Methods : 215 procedures were performed from November 2007 to August 2018.

Data was collected from medical charts and endoscopic data was collected from endoscopic reporting system. Patient backgrounds, type of FB, location, procedure time, type of procedure, type of removal device, success and technical complication were reviewed and analyzed retrospectively.

Results : 109(51\%) procedures were performed in older adults. The most common type of FB was press-through-pack (PTP). Most common location was the esophagus $(60.5 \%)$. Transparent caps with grasping forceps were the most common used devices $(38.1 \%)$. The success rate was in all cases $(100 \%)$ and only one complication case $(0.5 \%)$.

Conclusions : Endoscopic management of foreign bodies in our Center was safe and effective. The devices used for the FB extraction were depending on the location and type of FB. able forceps for difficult endoscopic tissue samplings (with Video)

Department of Gastroenterology, SMG-SNU Boramae Medical Center, Seoul National University College of Medicine

ODong Seok Lee, Su Hwan Kim, Ji Won Kim

AIMS OF THE STUDY : Biopsies with conventional forceps beyond the operating range are difficult and cumbersome. Thus, we developed a new forceps and evaluated its technical feasibility.

METHODS : A bendable forceps was constructed with two channels and a two-stage knob. The bending motion-related structures were designed to improve the range of motion. For performance comparisons, we established a scoring system for the evaluation of the forceps, with 9 special cases. All procedures were performed by 5 expert endoscopists.

RESULTS : Biopsies with the bendable forceps were easy and faster than those performed with conventional forceps in the 9 cases. The mean evaluation scores were $6.2 \pm 0.4$ and $1.96 \pm 0.2$ for the bendable and conventional forceps, respectively $(\mathrm{P}<0.001)$.

CONCLUSIONS : This preliminary study showed that the newly developed bendable forceps allowed for a wide range of motion and is more effective than conventional forceps for endoscopic biopsies in complex GI lesions.

\section{Q IP-68_E_ Percutaneous Endoscopic Gastrostomy Re-}

Division of Gastroenterology, Department of Internal Medicine, Inha University Hospital

OJung Hwan Lee, Gye Sook Kwon, Hyungkil Kim, Yong Woon Shin

\section{Purpose}

Percutaneous endoscopic gastrostomy (PEG) feeding provides enteral nutrition to patients with neurological dysphagia. However. the effect of PEG on the prevention of aspiration pneumonia is controversial. The purpose of this study is to evaluate the effect of PEG on neurological disability patients experiencing pneumonia

\section{Methods}

A retrospective review was performed for 238 patients who underwent PEG from January 2008 to December 2018. Of these patients, 52 patients had occurred pneumonia within 2 years before PEG. Among them, 23 Patients without enough follow-up period were excluded. The episodes and 6-months incidence of pneumonia were compared before and after PEG.

\section{Results}

Median follow-up of 29 patients after PEG is 20.0 months (interquartile range [IQR ], 14.0-32.6 months). Eleven patients developed pneumonia during nasogastric tube feeding prior to PEG insertion. $\mathrm{Pa}$ tients experienced significantly fewer episodes of total pneumonia after PEG (median 2 (IQR 1-2) vs 1 (IQR 0-1) episodes, $P<0.001$ ). 6 -months incidence of pneumonia after PEG was decreased than that of total pneumonia (median 1.70 (IQR 0.92-4.19) vs 0.18 (IQR $0-0.34$ ) days, $P<0.001)$ and pneumonia associated with nasogastric tube feeding (median 0.83 (IQR 0.58-2.65) vs 0.22 (IQR 0-0.55) days, $P=0.008)$

\section{Conclusion}

PEG can also prolong interval of pneumonia, even for nasogastric tube feeding patients. However, large prospective studies will be needed.

Percutaneous endoscopic gastrostomy Pneumonia |P_69_E_ $\begin{aligned} & \text { Efficacy of endoscopic vacuum assisted closure } \\ & \text { treatment for postoperative anastomotic leak of }\end{aligned}$ gastric cancer

Yonsei University College of Medicine OJun Chul Park, Soo In Choi, Eun Hye Kim, Sung Kwan Shin, Sang Kil Lee, Yong Chan Lee

Background : Endoscopic vacuum-assisted closure (EVAC) has been attempted as a new nonsurgical treatment option for leakage. We aimed to evaluate clinical outcomes of EVAC and to compare its efficacy with that of self-expandable metallic stent (SEMS) for postgastrectomy leakage.

Patients : Between 2007 and 2018, 39 patients underwent endoscopic treatment for anastomotic leakage after gastrectomy for gastric cancer. Of them, 28 patients were treated with SEMS only, 7 with EVAC after SEMS failure, and 4 with EVAC only. We compared the characteristics and outcomes between EVAC and SEMS.

Results : Median follow-up duration was 17 (range 0-48) months. All EVAC cases healed successfully, and 1 case of anastomotic stricture occurred within 1 year. By comparison, treatment failure occurred in 2 SEMS cases $(7.1 \%)$ and 4 cases $(4.3 \%)$ of anastomotic stricture occurred within 1 year. Median treatment duration of EVAC (15 [range 6-47] days) was shorter than that of SEMS (36 [range 7-108] days). Relatively large leaks were treated successfully with EVAC (EVAC : median 2.1 [range 1.5-3.3] cm, SEMS : 1.0 [range $0.2-2.5] \mathrm{cm})$. Median weight loss after therapy was similar for EVAC $(8 \mathrm{~kg})$ and SEMS $(9 \mathrm{~kg})$.

Conclusions : EVAC can be effective for postgastrectomy anastomotic leakage. A large leak size could be an important clinical factor for considering EVAC as a treatment option.

Vacuum-assisted Closure Anastomotic Leak 


\section{IP-70_S}

Prognostic significance of serum alkaline phosphatase level in patients with unresectable advanced gastric cancer

Department of Surgery, Kochi Medical School ${ }^{1)}$, Department of Human Health and Medical Sciences, Kochi Medical School ${ }^{2)}$ OTsutomu Namikawa ${ }^{1)}$, Nobuko Ishida ${ }^{1)}$, Keiichiro Yokota ${ }^{1)}$, Sachi Tsuda $^{1)}$, Kazune Fujisawa ${ }^{1 /}$, Eri Munekage ${ }^{1 /}$, Jun Iwabu ${ }^{1)}$, Masaya Munekage $^{1)}$, Sunao Uemura ${ }^{1)}$, Shigehiro Tsujii ${ }^{1)}$, Hiromichi Maeda ${ }^{12}$, Hiroyuki Kitagawa ${ }^{12}$, Michiya Kobayashi ${ }^{2)}$, Kazuhiro Hanazaki ${ }^{1)}$

Backgrounds : This study evaluated the prognostic value of alkaline phosphatase (ALP) and lactate dehydrogenase (LDH) together with host-related factors in patients with unresectable advanced gastric cancer.Methods : The study enrolled 262 patients who received chemotherapy for unresectable advanced gastric cancer at our institution from 2007 to 2015. Clinicopathological information and systemic inflammatory response data were analyzed for associations between baseline cancer-related prognostic variables and survival outcomes.Results : The median survival time (MST) was significantly lower for patients with high ALP, high LDH, high total bilirubin, high aspartate aminotransferase, high alanine transaminase, high gamma-glutamyltransferase, high creatinine, a Glasgow prognostic score (GPS) of 1 or 2 score compared to GPS 0 , higher compared to lower neutrophil to lymphocyte ratio (NLR) 3.9, lower compared to higher prognostic nutrition index 36.1, T3-4 compared to T1-2 tumor and diffuse-type compared to intestinal-type histology. Multivariate survival analysis identified high ALP 322 (HR 1.808; $\mathrm{P}=0.044)$, T2-3 (HR 2.622; $\mathrm{P}=0.013)$, and diffuse-type gastric cancer (HR 2.325; $\mathrm{P}=0.003$ ) as significant independent predictors of worse prognosis in the studied group of cancer patients. Conclusions: High levels of ALP is an independent, worseprognosis factor for patients receiving chemotherapy for unresectable and recurrent gastric cancer. ences of laparoscopic sleeve gastrectomy for morbid obesity

Department of Surgery, Nagasaki University

OAkira Yoneda, Kengo Kanetaka, Shinichiro Kobayashi,

Yusuke Inoue, Yasuhiro Torashima, Shinichiro Ito, Mitsuhisa Takastuki, Susumu Eguchi

(Introduction)

Laparoscopic sleeve gastrectomy (LSG) is one of the most preferred treatment option for morbid obesity. In 2014, We introduced laparoscopic sleeve gastrectomy for treatment of patients with morbid obesity at our Institution. The aim of this study was to assess the safety and short-term efficacy of LSG.

\section{(Methods)}

A retrospective analysis of the 18 patients who underwent LSG between 2014 and 2019 was performed. We evaluate operative time blood loss, postoperative complications, length of hospital stay, debree of weight and HbAlc reduction and changes in food prefergree of
ences.

\section{(Results)}

Our 18 morbidly obese patients, who underwent LSG had an average preoperative BMI 43.2 (35.0 to $60.2 \mathrm{~kg} / \mathrm{m} 2$ ) and average $\mathrm{HbA}$ 1c $7.2 \%$. There were 11 men and 7 women, with mean age 39.8 (30 to 50 years) Mean operative time and blood loss were 234 (range 169-292 mins) and $14(0-50 \mathrm{ml})$ respectively. We had no conversion to open proce to open procedure. There was no postoperative complication. Me EWL and $H$ EWL and $\mathrm{HbA} 1 \mathrm{c}$ at 1 year were $65.2 \%$ and $5.7 \%$ respectively. Food preference showed that sweet and salty foods intake was not changed.

(Conclusions)

In our initial experience, LSG is a safe and effective treatment option for the obese patient. Follow-up will be necessary to evaluate long-term results.

IP-72_S

Identification of novel plasma microRNAs, predicting and modulating the effect of ; chemotherapy in gastric cancer

Division of Digestive Surgery, Kyoto Prefectural University of Medicine

OKeiji Nishibeppu, Shuhei Komatsu, Takuma Kishimoto, Jun Kiuchi, Toshiyuki Kosuga, Hirotaka Konishi, Atsushi Shiozaki, Kazuma Okamoto, Hitoshi Fujiwara, Eigo Otsuji

\section{BACKGROUND :}

This study aimed to explore microRNAs (miRNA) in plasma as novel biomarkers for chemosensitivity in gastric cancer (GC). We have identified cancer-associated plasma microRNAs, which could be useful as biomarkers for cancer detection, predicting prognosis and chemosensitivity in digestive tract cancers.

\section{MATERIALS :}

Using microRNA array-based approach, we selected miR-X which enhance chemoresistance to 5-FU. We also selected miR-Y which enhance chemosensitivity to 5-FU, using a systematic review of the NCBI database. We compared preoperative plasma miR levels between patients with or without recurrences. All patients underwent an adjuvant chemotherapy regimen with S-1.

\section{RESULTS :}

1) Plasma miR-X was up-regulated in GC patients with recurrence compared to GC patients without, and plasma miR-Y was downregulated in GC patients with recurrence compared to GC patients regulated in GC patients with recurrence compared to GC patients
without. 2) After overexpressing miR-X in GC cells, miR-X induced without. 2) After overexpressing $\mathrm{miR}-\mathrm{X}$ in GC cells, miR-X induced
a significant chemoresistance to 5 -FU, up-regulating TS, DPDin via significant chemoresistance to 5-FU, up-regulating TS, DPDin vi-
tro and vivo analyses. 3) After overexpressing miR-Y in GC cells, miR-Y enhanced the effect of 5-FU, inducing apoptosisand downregulating TS in vitro analyses. In vivo, miR-Y induced a significant chemosensitivity to $5-\mathrm{FU}$.

CONCLUSIONS : Plasma miR-X and $\mathrm{Y}$ could be biomarkers as a liquid biopsy for predicting chemosensitvity in GC, respectively. Furthermore, the miR-Y as a 5-FU modulator could be a novel agent for anti-cancer therapy.

biomarker

chemosensitivity

IP-73_S Equivalent feasibility and safety of perioperative care by ERAS in open and laparoscopyassisted distal gastrectomy for gastric cancer : A single-institution ancillary study using the patient cohort enrolled in the JCOG0912 phase III trial

Department of Gastrointestinal Surgery, Kanagawa Cancer Center ${ }^{1}$, Department of Surgery, Yokohama City University ${ }^{2)}$ OToru Aoyama ${ }^{1.2)}$, Tsutomu Hayashi ${ }^{1 /}$, Tsutomu Sato ${ }^{1)}$, Takanobu Yamada ${ }^{1)}$ Takashi Ogata ${ }^{1)}$, Haruhiko Cho ${ }^{1)}$, Takaki Yoshikawa ${ }^{1)}$

Background : Laparoscopy-assisted distal gastrectomy (LADG) has an advantage of earlier recovery after surgery due to having lower invasiveness and wound pain than open distal gastrectomy (ODG). However, whether or not the same enhanced recovery after surgery (ERAS) program for LADG is equally feasible and safe for ODG remains unclear. Methods: We retrospectively extracted the clinical data of the patients enrolled in JCOG0912 from the medical record system of our hospital and compared the treatment process and short-term surgical outcomes between LADG and ODG. The morbidity was defined as complications of grade 2 or more. Results : One hundred and sixty-three patients were entered from our hospital and randomized to undergo ODG (82 patients) or LADG (81 patients). The patient's backgrounds were similar between the ODG and LADG groups. The rate of completing the clinical pathway was $95.1 \%$ in both groups, and the rates of completing each ERAS element were similar. The median hospital stay after surgery was 9 days in both groups. Morbidity, defined as ClavienDindo classification more than grade 2 , was observed in $6.1 \%$ of the ODG group and $11.1 \%$ of the LADG group. No mortality occurred in either group. Conclusion : This study showed that the regimen of perioperative care performed by the ERAS program for LADG was equally feasible and safe for ODG with additional pain control. 
|P_74S The strategy for IDI (Iatrogenic duodenal Injury)

Division of Digestive Surgery, Kyoto Prefectural University of Medicine

OHisashi Ikoma, Michihiro Kudo, Katsutoshi Shoda, Tomohiro Arita, Toshiyuki Kosuga, Hirotaka Konishi, Yasutoshi Murayama, Atsushi Shiozaki, Yoshiaki Kuriu, Ryo Morimura,

Takeshi Kubota, Masayoshi Nakanishi, Hitoshi Fujiwara,

Kazuma Okamoto, Eigo Otsuji

Background : Iatrogenic duodenal injury : IDI is a severe complication caused by endoscopic operation or surgery. In this presentation, we will describe the strategy for IDP based on our experience. Methods and Materials: We retrospectively reviewed 11 cases that suffered from IDI at our institution between 2003 and 2018. Results : Endoscopic retrograde cholangiopancreatography : ERCP caused IDI in 5 cases, endoscopic submucosal dissection : ESD in 3 cases, and urgical injury in 3 cases respectively. Four surgical procedures including simple suture, utilizing $\mathrm{T}$ tube, gastrojejunostomy and were performed to repair IDP. As for the duodenojejunostomy, there were no severe complications among the patients who underwent patching or anastomosis to the injured site utilizing jejunum as a material for reinforcing the repaired portion. In the case of using $\mathrm{T}$ tube had required reoperation and long-term treatment. Finally, all patients had been discharged. We emphasize following points according to the procedure for repairing IDI. 1 : to reduce the tension applied to the repaired site as possible $2:$ to secure effective drainage 3 : to remove fat tissue around the right kidney after resecting the Gerota's fascia. We found those issues were crucial in the management for IDI through our experience. Conclusion : IDI is a severe disease, so we should pay paramount attention preventing the complications.

Iatrogenic duodenal injury duodeno-jejunostomy 


\section{IP-75 C}

Education and engagement : Key factors enabling colorectal cancer patients to promote benefits of screening amongst their siblings

Division of Colorectal Surgery, University Surgical Cluster

Dedrick Kok Hong Chan, Tian Zhi Lim, Melody Chow, Emily Chew, Carol Yap, Yi Xuan Lim, Ker Kan Tan

\section{Background}

The study aims to determine a novel counselling package for colorectal cancer patients to advocate screening amongst their siblings.

\section{Methods}

Colorectal cancer patients from a tertiary centre were assigned to receive a new counselling package versus the current standard advice. Block randomisation with participants blinding, and analysis and sibling response rates.

\section{Results}

Between 1 May 2017 to 30 Apr 2018, 83 participants received the new $(n=40)$ or standard $(n=43)$ counselling package. The sample demographics were comparable.

Despite the intervention, both arms had similar sibling contact rates $(>90 \%)$ and preferably to inform their siblings in person. Given the frequent engagement and education in the intervention group, the higher level of assumed screening awareness has in creased in the likelihood ( $\mathrm{p}$-value : 0.039) of patients convincing their siblings to contact the study team. These siblings adopted a proactive approach towards contacting the study team (p-value : 0.009 ) and registered their interest to understand more about colorectal cancer screening. However, the uptake of screening colonoscopy remained dismal in both arms.

\section{Conclusion}

The novel counselling package increases the awareness and importance of colorectal cancer screening amongst patients enabling them to advocate screening amongst their siblings. Further inter ventions are required to overcome the barriers towards uptake of colonoscopy.

\section{COLORECTAL CANCER SCREENING}

IP-76_C

Improvement of Awareness And Knowledge in Colorectal Cancer and Its Screening Using Online Learning

Universiti Sultan Zainal Abidin

ONurulhuda M. Hassan, Norwati Daud, Aniza A. Aziz, Harmy M. Yusof

Background and Aims : Colorectal cancer is the third most common cancer worldwide with 1.8 millon new cases in 2018. Colorectal cancer screening activities are found be be effective in reducing the mortality related to colorectal cancer. However, the uptake of these activities are still unsatisfactory, with one of the cause being low awareness and knowledge regarding the cancer and its screening. The aim of this study was to introduce a multimodality online course on colorectal cancer and its screening ; and to measure its effectiveness in providing the awareness and knowledge in colorectal cancer and its screening. Methodology : An online course was designed for general public, incorporating essential knowledge regarding colorectal cancer and its screening using open courseware. Random group sampling was done to sample 80 undergraduate students. Knowledge and awareness on colorectal cancer and its screening was measured using a validated questionnaire pre- and post-exposure to the online course. Paired t-test in SPSS ver22 was used for analysis. Results : There was significant difference in Preand Post-exposure test on overall knowledge $(p<0.001 ; \mathrm{CI}$ : $14.23,18.78, \mathrm{t}=14.51)$, knowledge on colorectal cancer screening ( $\mathrm{p}$ $<0.001 ; \mathrm{CI}: 3.04,4.46, \mathrm{t}=10.62)$, and awareness ( $\mathrm{p}=0.001 ; \mathrm{CI}$ : $3.41,5.26, \mathrm{t}=9.39)$. Conclusion : Multimodality online learning is a useful tool to increase awareness and knowledge regarding colorectal cancer and its screening and is feasible to be used.

IP-77_C

Applicability of Colorectal cancer screening guidelines to Asians in the United States

Mayo Clinic

OVeeravich Jaruvongvanich

\section{Objective}

American Cancer Society recommended colorectal cancer (CRC) screening begin at age 45 rather than 50 . However, it is unclear if this recommendation would be applicable to Asians in the US

Methods

CRC incidence rates during 2000-2015 were obtained from 18 Surveillance, Epidemiology and End Results registries. Incidence rates were age adjusted by the direct method to the 2000 US standard population

\section{Results}

CRC incidence trends in non-Asians aged $45-50$ (by 1.2\% per year) were higher than Asians aged $45-50$ (by $0.1 \%$ per year) with the incidence rate of 34 versus 26.8 per 100,000 people, respectively. When compared to Asians aged 50-

55 , Asians aged $45-50$ had a lower incidence rate with stable incidence trend.

\section{Conclusions}

Asians aged 45-50 with CRC has fairly stable incidence rate and the rate is lower than Asians aged 50-55 and non-Asians aged 45-50. CRC screening recommendations may not be applicable across to Asians in the US.

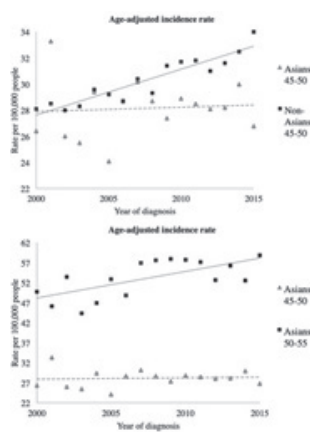

Colorectal cancer screening United States

IP-78_C A simple phone call with the right siting of care : The means to complete the evaluation of patients with positive FIT results.

Division of Colorectal Surgery, University Surgical Cluster Dedrick Kok Hong Chan, Tian Zhi Lim, Yi Xuan Lim, Carol Pei Ling Yap, Ker Kan Tan

\section{Background}

We aim to explore the issues hindering patients with a positive FIT from undergoing screening colonoscopy.

\section{Methods}

Participants were randomly selected from a FIT database. A prospective qualitative design using semi-structured open ended questions through a phone interview.

\section{$\underline{\text { Results }}$}

Sixty-five patients with positive results were approached. The sample was primarily male $(62.5 \%$ ) with a median age of 66 (range, 62 84 , years). Majority Chinese $(96.9 \%)$, living in public housing $(81.3 \%)$ and conversed in English $(78.1 \%)$. The median time from test to receiving a call was 12 (range, $3-28$, weeks). At time of interview, almost half $(n=30,46.2 \%)$ had received medical consultation. Those without any medical consultation (53.8\%), barriers precludThose without any medical consultation (53.8\%), barriers preclud-
ing its uptake include 1) lack of knowledge over the interpretation of a positive FIT result, 2) denial, 3) inconvenience to undergo further investigations, 4) cost and 5) failure to receive follow-up reminders.

About half $(48.6 \%)$ of the participants who did not undergo any medical consultation initially agreed to receive consultation after receiving a simple phone call enquiry. The converts preferred their follow-up near their home.

\section{Conclusion}

With the provision of timely and appropriate reminders and advice, the uptake of screening colonoscopy for patients with positive FIT can be maximised. 
Q IP-79 C Rectal carcinoids: Is surveillance neces-

National University Hospital System

OAnn Su Lui, John Jian-Xian Quek, Frederick Hong Xiang Koh,

Dedrick Kok Hong Chan, Kan Ker Tan

Rectal carcinoids make up about $1-2 \%$ of all rectal tumours. There is currently no consensus on its subsequent management and follow-up. This study aims to compare the outcomes of various management options for rectal carcinoids at a single institution in Singapore.

All patients who were diagnosed with rectal carcinoids between February 2014 and July 2016 in a single tertiary academic institution were reviewed. Data collected include their clinicopathological characteristics, intervention undertaken, and outcomes.

During the study period, 24 patients, median age 58-years-old (21$74)$, were diagnosed with rectal carcinoids. Rectal bleeding (38\%) was the most common presenting symptom, while $25 \%$ were diagnosed incidentally during screening colonoscopy.

Eighteen patients were diagnosed to have rectal carcinoids after snaring polypectomy, and no further intervention was performed Six patients underwent local resection either by means of trans-anal excision of the tumour $(12.5 \%)$ or endoscopic submucosal resection (ESD) $(12.5 \%)$. All rectal carcinoids identified were low-grade.

$50 \% \quad(n=9)$ of the patients who only underwent polypectomy had margins involved by carcinoid but none recurred after a median follow-up of 19 months (0-41 months). Only one patient who underwent ESD had a low-grade recurrence. There were no deaths in our study group throughout our follow-up period.

Local excision of tumour including polypectomy is sufficient for lowrisk rectal carcinoids. Surveillance colonoscopy can be avoided if tumour has been excised with clear margins.

Rectal carcinoid Surveillance 


\section{IP-80_E}

22g Franseen needle vs $19 \mathrm{~g}$ standard needle for tissue acquisition during EUS-FNA for pancreatic tumor

First Department of Internal Medicine, Gifu University Hospital OTakuji Iwashita, Shinya Uemura, Masahito Shimizu

Tissue acquisition during EUS-FNA for pancreatic tumors (PT) is important for evaluation of immunostaining or tissue architecture to diagnose specific diseases and its importance is increasing due to expansion of precision medicine. Recently, 22g Franseen needle (22 FR) emerged as histology needle.

Aims : To compare 22FR with $19 \mathrm{~g}$ standard needle (19ST) for tissue acquisition during EUS-FNA for PT using propensity score matching (PSM).

Methods : A retrospective database analysis found 434 patients who underwent EUS-FNA for PT $(\geq 20 \mathrm{~mm})$ using 22FR or 19ST between 2009 and 2018. PSM formed the matched 158 patients. The endpoints were diagnostic accuracy for malignancy in histology alone and combined histology and cytology, histological assessment score ranged 0 to 5 (full histological specimen), and safety.

Results : Baseline characteristics of 22FR group $(n=79) / 19 S T$ group $(\mathrm{n}=79)$ were age $[$ yo, median (range) ], 70 (39-90) /71 (2788) ; gender (female), 40/38; tumor size (mm), 27 (20-91) /31 (20-65) ; location, (head) $42 / 43$, (body/tail) $37 / 36$; scope location, (stomach, D1, D2) 42/43, 22/24, 15/12 and showed no significant difference in any factors. Final diagnoses were malignancy $(68 / 67)$ and benign $(11 / 12)(\mathrm{p}=0.82)$. Histological analysis showed accuracy of $99 \% / 90 \%(\mathrm{p}=0.01)$ and combined histological and cytological analysis showed accuracy of $99 \% / 92 \%(\mathrm{P}=0.05)$. Histological assessment scores per pass analysis $(0,1,2,3,4,5)$ were $7 / 12,0 / 5,5 / 19,6 / 18,22$ / $25,140 / 108(\mathrm{p}<0.0001)$. Adverse events were seen 2 patients in each group $(2.6 \% / 2.6 \%: \mathrm{p}=0.99)$.

Conclusion : 22FR during EUS-FNA for PT might improve quality of histological specimen and histological diagnostic accuracy in comparison with 19ST.

\section{\&IP-81_E}

Endoscopic Ultrasound (EUS) guided biliary drainage performed by non-EUS expert ; a lesson learned from a rural hospital in Thailand

Department of Internal Medicine, Rajavithi Hospital ${ }^{1)}$, Department of Surgery, Thabo Crown Prince Hospital ${ }^{2)}$

Tanyaporn Chantarojanasiri ${ }^{1}$, Chaloermphon Boonmee ${ }^{2)}$,

Pacharin Soimeesaeng ${ }^{2)}$, Wattana Pareesri ${ }^{2)}$

Introduction

Endoscopic ultrasound guided biliary drainage (EUS BD) an alternative therapy after fail ERCP. Apart from being technically demanding the need for procedure is unpredictable. We reported cases series of EUS-BD performed as a rescue therapy by non-EUS expert.

Material and method

EUS BD performed in a 150-bed rural hospital, which has 500 ERCPs per year. EUS BD was performed after failed ERCP for palliative drainage for malignant biliary obstruction, by endoscopists who experienced more than 1000 cases of ERCP but less than 50 cases of EUS FNA. Surgical and interventional radiology back up were prepared for every patients.

Results

Totally 29 cases of EUS BD was performed in patients diagnosed as malignant biliary obstruction with failed ERCP. Procedure time was
33 minutes (27-38). Technical success rate was $100 \%$ and clinical 33 minutes (27-38). Technical success rate was $100 \%$ and clinical
success rate was $79 \%$. Immediate complications were stent migra-
tion in 1 cases and bile leakage in 2 cases. Procedure-related complition in 1 cases and bile leakage in 2 cases. Procedure-related complications were experienced in $15^{\text {th }}$ and $20^{\text {th }}$ case. Late complications were stent obstruction in $4 / 29$ and cholangitis in $9 / 29$. None of drainage.

Conclusion

EUS BD performed by non-EUS expert might be feasible in selected conditions given the presence of surgical and interventional radiology back up.

EUS guide biliary drainage malignant biliary obstruction
IP_82 E Factors Predicting Poor Clinical Outcomes in Patients with ERCP-related Perforation

Konkuk University Medical Center ${ }^{1)}$, Department of Internal Medicine, Asan Medical Center ${ }^{2)}$

$\bigcirc$ Tae Yoon Lee ${ }^{1)}$, Myung Hwan Kim

Background : Perforation is a very serious complication resulting ERCP. There has been no study of the predictive factors for severe clinical outcomes in patients with ERCP-related perforation.

Objective : To investigate the factors predictive of severe clinical outcomes of ERCP-related perforation.

Design : Retrospective cohort study.

Patients : We assessed the clinical outcomes of 15,452 ERCP procedures performed on 9,295 patients from 2009 to 2013. For those patients who suffered ERCP-related perforation, clinical information from before the ERCP was obtained from a constructed ERCP database. The locations of air densities were derived from radiologic images.

Main Outcome Measurements : Independent predictive factors of severe clinical outcome in patients with ERCP-related perforation.

Results : ERCP-related perforation occurred in 55 of the 15,452 $(0.4 \%)$ procedures. Five were scope-related and the other 50 were scope-unrelated. Of the latter, $12(24.0 \%)$ led to severe clinical outcomes, and all five scope-related perforations required surgery. In scope-unrelated perforations, extra-abdominal air (odds ratio [OR], $6.07 ; 95 \%$ confidence interval [CI $], 1.02-37.39)$ and rebound tenderness (OR, 11.45; 95\% CI, 1.37-95.77) were significantly associated with severe clinical outcome.

Conclusion : Scope-related perforation of the ERCP is a serious complication requiring surgery. In scope-unrelated perforations, extra-abdominal air and rebound tenderness are predictive of more severe clinical course.

Cholangiopancreatography Intestinal Perforation
$14 \mathrm{~mm}$ uncovered versus $10 \mathrm{~mm}$ covered metal stent for distal biliary obstruction : a multicenter retrospective study

Division of Endoscopy, Shizuoka Cancer Center ${ }^{1)}$, Department of Gastroenterology, Shizuoka General Hospital ${ }^{2}$

Tatsunori Satoh ${ }^{11}$, Hirotoshi Ishiwatari ${ }^{11}$, Shinya Kawaguchi ${ }^{2}$

Background and Aim : In patients with unresectable distal malignant biliary obstruction, endoscopic biliary drainage by using a metal stent (MS) is an established palliative treatment. To prevent recurrent biliary obstruction (RBO), a $14 \mathrm{~mm}$ diameter uncovered MS (14-UMS) was developed. However, there is no study comparing 14-UMS to different types of MSs. Therefore, we retrospectively compared clinical outcomes of 14-UMS with those of $10 \mathrm{~mm}$ diameter covered MS (10-CMS).

Method : Between 2013 November and 2018 December, 222 pancreatic cancer patients with malignant distal biliary obstruction who underwent 14-UMS $(\mathrm{N}=83)$ or 10 -CMS $(\mathrm{N}=139)$ placement at two tertiary-care centers were included and analyzed retrospectively in the study.

Result: There was no significant difference between the two groups in the patient characteristics and overall survival. Median time to RBO (TRBO) did not differ significantly between the 14UMS and 10 - CMS groups (not reached vs 308 days, $P=0.12$ ). RBO occurred in $16(19 \%)$ and $43(31 \%)$ in the 14-UMS and the 10-CMS, respectively $(P=0.061)$. By multivariate analysis, a 10-CMS placement was an independent risk factor for RBO $(P=0.041)$.

Conclusion: There was no significant difference between two groups in terms of TRBO. However, the rate of RBO had a lower tendency in the 14-UMS and a 10-CMS placement was an independent risk factor for RBO, indicating the 14-UMS can be a promising MS. 

IP-84_E The safety and efficacy of a large-bore full covobstruction

Department of Internal Medicine, Gangnam Severance Hospital, Yonsei University College of Medicine

OSung Ill Jang, Yonsoo Kim, Sunghwan Yoo, Dong Ki Lee

Objective : In patients with unresectable malignant biliary obstructions, self-expanding metallic stent (SEMS) is widely used. However, SEMS is susceptible to occlusions by tumor ingrowth, outgrowth, and sludge formation. To overcome these adverse effects, we developed a large-bore dumbbell-shaped full covered SEMS (FCSEMS-L) and evaluated the efficacy and safety of FCSEMS-L.

Methods : Patients with unresectable distal malignant biliary obstruction was retrospectively enrolled. A total of 90 patients were included. A FCSEMS-L was inserted endoscopically in 46 patients, and a FCSEMS-C was inserted in 44 patients. Clinical characteristics, complications, and prognosis were also analyzed.

Results : Stent occlusion occurred in 7 patients (15.2\%) who received FCSEMS-L and in 18 patients (40.9\%) who received FCSEMS-C $(p=0.027)$. Stent occlusion due to sludge impaction was occurred in 3 patients with FCSEMS-L, and occurred in 13 patients with FCSEMS-C $(p=0.018)$. There are no difference in mean followup period (FCSEMS-L 193.2days vs FCSEMS-C 278.8 days, $\mathrm{p}=$ $0.236)$, stent migration $(\mathrm{p}=0.559)$, and cumulative stent patency ( $\mathrm{p}$ $=0.078$ ). Complications, including cholangitis and pancreatitis, were found to be acceptable and resolved by conservative management in both groups.

Conclusions : Although the use of a FCSEMS-L produced no significant differences in stent patency or stent migration rate, the FCSEMS-L can be used safely in the human bile duct and prevent stent occlusion effectively due to sludge impaction.

Malignant biliary obstruction large bore dumbbell-shaped FCSEMS IP-85_E A short length stent with an anti-migration deary anastomotic stricture after a biliary operatio

Department of Internal Medicine, Gangnam Severance Hospital, Yonsei University College of Medicine ${ }^{1)}$, Department of Radiology, Gangnam Severance Hospital, Yonsei University College of Medicine $^{2)}$

OSung Ill Jang ${ }^{1)}$, Tae Ryong Chung ${ }^{1)}$, Sunghwan Yoo ${ }^{1)}$, KwangHun Lee $^{2)}$, Seung-Moon Joo ${ }^{2)}$, Jung Hye Choi ${ }^{1 /}$, Dong Ki Lee ${ }^{1}$

Objective : In the treatment of anastomotic biliary stricture (ABS), fully covered self-expandable metal stent (FCSEMS) is useful. In this study, we investigated the efficacy of modified short removable FCSEMS with an anti-migration design for high-level ABS.

Methods : FCSEMS insertion was performed endoscopically on patients with high-level ABS after a biliary operation. The FCSEMS was maintained initially three months and exchanged every three months until the stricture was resolved. After removal of the FCSEMS, complications and recurrence were accessed during follow-up.

Results : A total of 71 patients with a median age of 58 years. The types of the previous operation were living donor liver transplantation $(77.5 \%)$, deceased donor liver transplantation $(8.5 \%)$, liver lobectomy $(11.3 \%)$ and cholecystectomy alone $(2.8 \%)$. The technical success rate was $100 \%$, and the clinical success rate was $95.7 \%$. The recurrence rate was $22.5 \%$, and the complication rate was $14 \%$. All the stents were removable, and stent migration was noted in 4 patients $(5.6 \%)$.

Conclusions : The newly designed FCSEMS is a potentially feasible and effective for the treatment in patients with high-level ABS after a biliary operation.

post-operative biliary stricture modified fully covered self-expandable metal stent 


\section{$\star$ IP-86_H}

SVR to DAA therapy in active HCC, inactive HCC, and non-HCC HCV patients : a propensity score matched (PSM) study from the Asia Liver Consortium

Department of General Internal Medicine, Kyushu University Hospital $^{1)}$, Department of Gastroenterology, Ogaki Municipal Hospital $^{2}$, Department of Virology and Liver Unit, Graduate School of Medical Sciences, Nagoya City University ${ }^{3)}$, Department of Hepatology, Osaka City University Medical School ${ }^{4}$, Liver Center, Saga University Hospital ${ }^{5}$, Department of Gastroenterology, Yamagata University ${ }^{6}$, GI/Hepatology, Stanford University ${ }^{7)}$ $\bigcirc$ Eiichi Ogawa ${ }^{1)}$, Hidenori Toyoda ${ }^{2)}$, Etsuko Iio ${ }^{3)}$, Masaru Enomoto $^{4)}$, Shinji Iwane ${ }^{5)}$, Toshifumi Tada ${ }^{2}$, Satoshi Yasuda ${ }^{2}$, Hirokazu Takahashi ${ }^{5}$, Yuichiro Eguchi ${ }^{5)}$, Yoshiyuki Ueno ${ }^{6}$, Akihiro Tamori, ${ }^{4}$, Norihiro Furusyo ${ }^{1)}$, Yasuhito Tanaka ${ }^{3)}$, Mindie H. Nguyen $^{7}$

Aim : To evaluate DAA treatment outcomes in a large East Asian $\mathrm{HCV} / \mathrm{HCC}$ versus $\mathrm{HCV} /$ non-HCC patients.

Methods: We included 6.081 patients (465 HCC, 5,616 non-HCC) treated with IFN-free DAAs in Hong-Kong, Japan, Korea, and Taiwan. We used PSM to match HCC and non-HCC groups on multivariable Cox regression to adjust for residual confounders.

Results : PSM of the entire study population yielded 436 matched pairs with balanced characteristics. Overall SVR rates were similar in HCC $(92.7 \%)$ and non-HCC $(95.0 \%)$ groups $(\mathrm{p}=0.16)$, but lower in HCC $(92.7 \%)$ and non-HCC $(95.0 \%)$ groups $(\mathrm{p}=0.16)$, but lower
in active HCC $(93.7 \%)$ compared to active HCC $(85.5 \%)$ cases $(\mathrm{p}$ $=0.03)$. On multivariable analysis, active HCC was independently associated with lower SVRs compared to non-HCC (OR 0.34 $\mathrm{p}=$ 0.01 but not inactive HCC or DAA regimen. Adverse effects were similar in $\mathrm{HCC}$ and non-HCC patients.

Conclusion: Inactive and non-HCC patients had similar SVR to DAA. However, compared to non-HCC, active HCC (but not specific DAA regimen) independently predicted lower SVR.
Direct-acting antivirals
High efficacy and improvement in CPT class with Sofosbuvir/Velpatasvir plus Ribavirin for 12 weeks in patients with CPT C decompensated cirrhosis

Northwestern University ${ }^{1}$, Gilead Sciences Inc. ${ }^{2)}$

$\bigcirc$ Michael Charlton ${ }^{1)}$, Tomohiro Ueno ${ }^{2)}$, ChenYu Wang ${ }^{2}$

Background The ASTRAL-4 study demonstrated the efficacy and safety of SOF/VEL+RBV in patients with CPT B decompensated cirrhosis. The current study is the first to evaluate the efficacy and safety of SOF/VEL+RBV in patients with CPT C decompensated cirrhosis. Methods This was an open-label, single-arm study conducted in France and the US. Patients had CPT score 10-12 at screening and infected with any HCV genotype. All subjects were treated with SOF/VEL+RBV for 12 weeks. The primary endpoint was SVR12 and Safety. Results Of the 32 patients enrolled, 26 (81\%) were male and $28(88 \%)$ were treatment naive. The mean age was 55 years. 18 patients had GT 1, 5 had GT 2, and 7 had GT 3. GT could not be determined for 2 patients. At baseline, CPT score ranged from 8-13, with 9 and 23 having CPT B and CPT C cirrhosis, respectively. MELD scores ranged from 10-25, 19 patients having scores $>15$. SVR12 was achieved in $78 \%$ of patients $(25 / 32)$, with no virologic failures. 3 patients received a liver transplant during the study. Among the 21 patients who achieved SVR12, who did not receive a liver transplant and who had CPT score evaluated, 48\% (10/ 21) improved in CPT class from baseline to post-treatment Week 12. $\mathrm{SOF} / \mathrm{VEL}+\mathrm{RBV}$ was well-tolerated with no Grade 3-4, SAEs, or deaths considered related to study drugs. Conclusions This study demonstrates that treatment with SOF/VEL+RBV for 12 weeks was well tolerated and resulted in high rates of SVR12 that were associated with clinical benefit, as measured by improvement in CPT class.

\section{IP-88_H}

$\mathrm{LDV} / \mathrm{SOF}$ is highly effective and safe in $\mathrm{Pa}$ tients with Chronic Hepatitis B Virus and Hepatitis C Virus Coinfection : Final Study Results

Chang Gung Memorial Hospital ${ }^{1}$, Gilead Sciences Inc. ${ }^{2}$ OTsung-Hui $\mathrm{Hu}^{1)}$, Hiroki Konishi2), ChenYu Wang ${ }^{2)}$

Background: Cases of HBV reactivation during HCV treatment have been reported in the IFN era and DAAs. This study evaluated the safety and efficacy LDV/SOF in patients with chronic HCV and HBV coinfection. Methods : 111 patients chronically infected with HCV GT 1 or 2 and HBV (positive for serum HBsAg) were enrolled into this study to receive LDV/SOF for 12 weeks. Following treatment, patients were monitored for safety (HBV DNA reactivation) and efficacy for a total of 108 weeks. HBV DNA reactivation is defined as a post-baseline increase from baseline to $\geqq$ LLOQ or $>1$ $\log 10 \mathrm{IU} / \mathrm{mL}$ in patients that were $<$ LLOQ or $\geqq$ LLOQ at baseline, respectively. Results : Baseline characteristics included : mean age 55 years, $62 \%$ female, $67 \%$ treatment naive, $84 \%$ without cirrhosis, $61 \%$ GT1, and HBeAg negative (99\%) with a mean baseline HBV DNA of $2.1 \log 10 \mathrm{IU} / \mathrm{mL}$. SVR12 was $100 \%$ and through week 96 of post treatment follow up ; there were no viral relapses. HBV DNA reactivation occurred in $73 \%$ of patients ; $12 \%$ of whom also had a corresponding ALT increase $>2 \mathrm{x}$ ULN. To date, 8 patients have started HBV therapy per Taiwanese treatment guidelines. The majority $(86 \%)$ of HBV reactivations occurred by post treatment week 12. $90 \%$ of HBV reactivations were asymptomatic with no patient developing clinical symptoms. Through 96 weeks of follow up, 19 patients had serious adverse events (AEs) ; 1 patient withdrew consent, and 2 patients died; none were considered drug related. Conclusions : LDV/SOF treatment remains a highly effective, safe and tolerable regimen in patients with $\mathrm{HCV} / \mathrm{HBV}$ coinfection.

HBV reactivation

Sofosbuvir

Q Associated Factors of Hepatitis C in Opioid Addicts Attending Therapy and Harmful Practices Related to Hepatitis Transmission

Universiti Sultan Zainal Abidin

ONurulhuda M. Hassan, Norwati Daud, Aniza A. Aziz

Aims : This study aims to determine the prevalence and associated factors of Hepatitis $\mathrm{C}$ in opioid addicts attending therapy and study the harmful practices related to its transmission.

Background : Opioid dependence, with an estimated prevalence of 16.5 million worldwide, carries a high cost to society by transmission of diseases such as Hepatitis C. Addicts at higher risk of Hepatitis $\mathrm{C}$ and its transmission should be identified upon entering therapy. Methods : This is a mixed-method cross-sectional study from June-July 2016. Addicts attending primary care therapy centres in Kuala Nerus district, Terengganu, Malaysia who fulfilled the inclusion and exclusion criteria and consented were included. A semistructured questionnaire was filled via face-to-face interview and reference to the case records was done. 122 questionnaires were assessed.

Results : $57 \%$ had Hepatitis C. Higher heroin use before therapy (p $=0.010, \mathrm{OR}: 3.57, \mathrm{CI}: 1.36,9.33$, relapse for heroin at 12 months of therapy ( $\mathrm{p}=0.004$, OR $: 4.14$, CI $: 1.56,10.95)$, and use of metamphetamine during therapy $(\mathrm{p}=0.031, \mathrm{OR}: 0.07, \mathrm{CI}: 0.01,0.78)$ were associated with positive Hepatits C status. $50.8 \%$ of those who relapsed still shared needle-washing containers despite non-needlesharing, all unaware that it may spread infections.

Conclusion: Education to avoid harmful practices which may spread hepatits $C$ is important even in addicts attending therapy, especially those who had higher heroin use, has relapses and still use metamphetamine during therapy. 

alysis

Chang Gung Memorial Hospital ${ }^{1}$, Gilead Sciences Inc. ${ }^{2)}$

$\bigcirc$ Tsung-Hui $\mathrm{Hu}^{1)}$, Tomohiro Ueno ${ }^{2)}$, ChenYu Wang ${ }^{2)}$

Background : Higher concentrations of the SOF metabolite, GS331007 , in severe renal impairment was reported. This study evaluated the safety and efficacy of LDV/SOF in HCV patients on dialysis. Method : HCV genotype 1, 2, 4, 5, or 6 patients, with or without compensated cirrhosis undergoing hemodialysis or peritoneal dialysis, were enrolled to receive LDV/SOF. Treatment duration was 8 , 12 or 24 weeks. The primary efficacy endpoint was the proportion of patients with SVR12. The primary safety endpoint was the proportion of patients who discontinued therapy due to AEs. Secondary endpoints included safety, viral resistance. Results : 95 patients were enrolled in Taiwan, Italy, Germany, the USA, and Belgium. The median age was 61 years (range $32-84$ ), 59\% were male, $22 \%$ treatment-experienced, and 20\% had cirrhosis. Most (92\%) were on hemodialysis with a mean dialysis duration of 11.5 years. There was no discontinuations due to AEs. To date, $87 / 95$ (92\%) of patients achieved SVR12 ; with no virologic failures. 4 patients died during therapy. None of the deaths were considered related to LDV/SOF. The most frequent AEs were muscle spasms (13\%), nasopharyngitis $(12 \%)$, and headache $(8 \%)$. Conclusion : Treatment with LDV/SOF in patients with and without cirrhosis undergoing dialysis resulted in a $92 \%$ SVR12 rate with no virologic failures. The regimen was well-tolerated with no treatment related discontinuations.

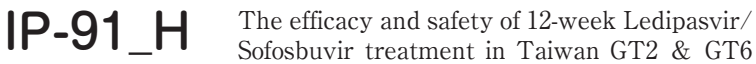
HCV patients : Real-world data from our hospital

Chi Mei Hospital, Liouying

OPei-Lun Lee, Jyh-Jou Chen, Hung-Da Tung, Chun-Ta Cheng, Tang-Wei Chuang, Hs u Kao, Yu-Hsun Wu, Mai-Gio Pang

Ledipasvir/Sofosbuvir has been approved for the treatment in HCV-2 patients in Taiwan, Korea, and Japan. However, the realworld evidences of LDV/SOF in GT2 HCV patients are limited. In addition, in Taiwan, the data of LDV/SOF in GT6 patients is still rare in real world. We aim to investigate the real-world efficacy and safety of 12-week LDV/SOF in GT2 and GT6 HCV patients by analyzing the data from our hospital.

There are 200 HCV-2 and 193 HCV-6 patients receiving LDV/SOF \pm RBV during 2018 and 2019 in our hospital. Among 200 GT2 patients, 73 patients were men, 16 patients were treatment experienced, and 31 patients were liver cirrhotic. 3 of them already completed follow-up duration, and all of them achieved SVR12. The other patients will have SVR12 results around Sep and will be reported in JDDW. For 193 GT6 patients, 84 patients were men, 11 patients were treatment experienced, and 70 patients were liver cirrhotic. The overall SVR12 was $96.8 \%$. During the treatment, the percentage of early discontinuation was 1\% for GT2 and 1.55\% for GT6 HCV patients.

In Taiwan real world, 12-week $\mathrm{LDV} / \mathrm{SOF} \pm \mathrm{RBV}$ treatment achieved high SVR12 in GT2 \& GT6 patients, and the efficacy is comparable to pivotal clinical studies. 


\section{IP-92_H}

Aspirin suppresses cell proliferation of hepatocellular carcinoma

Department of Gastroenterology and Neurology, Kagawa University

$\bigcirc$ Ting Ting Shi, Koji Fujita, Mai Nakahara, Hirohito Yone-

yama, Asahiro Morishita, Takako Nomura, Teppei Sakamato,

Kei Takuma, Hideki Kobara, Tsutomu Masaki

Background/Objective Hepatocellular carcinoma is the most common primary malignancy and the fourth leading cause of cancerrelated death. Aspirin is known to inhibit cell proliferation in a variety of cancer cells, including HCC. However, the mechanism remains unknown. We investigated the effects of aspirin on HCC in vitro and in vivo, and the study of microRNAs on antitumor effect. MethodsHuh7, Hep-G2, Hep-3B, Li-7, HLE and PLC/PRF/5 cell lines were used in this study by MTT assay to analysis the antiproliferation effect in vitro. Cell cycle and apoptosis of Huh7 in aspirin were analyzed by flow cytometric, ELISA, western blot and microRNA array. In vivo study was analyzed with a xenograft nude mouse model. Results Aspirin inhibited the proliferation of all the cell lines in our experiments in vitro. Additionally, aspirin induced $\mathrm{G}_{0} / \mathrm{G}_{1}$ cell cycle arrest of Huh7. This blockade was accompanied by a decrease in the levels of cyclin $\mathrm{E}$ and cell cycle-related proteins $\mathrm{CDK} 2$ and $\mathrm{Rb}$ phosphorylation. On the other hand, aspirin increased the level of caspase-cleaved cytokeratin 18, contributed to the induction of apoptosis. Aspirin reduced the EGFR activation in Huh7 cells, and the level of clusterin and HSP70. Furthermore, microRNA expression was markedly altered in Huh7 treated with aspirin. In vivo antitumor activity of aspirin caused a significant reduction in the growth of Huh7 tumors in nude mice. conclusion In conclusion, aspirin inhibited the growth of HCC cells by apoptosis, cell cycle arrest and alteration of microRNA in vitro and/or in vivo.

aspirin

hepatocellular carcinoma (HCC)

IP-93_H

Liver volume is an independent predictor of increased risk for hepatocellular carcinoma in patients with chronic viral hepatitis.

Department of Medicine, Seoul National University Bundang Hospital

OKyung Han Lee, Jin-Wook Kim

Background : Risk stratification is clinically mandatory in the process of hepatocellular carcinoma (HCC) surveillance. We previously showed that small-for-body build liver volume predicts increased risk of HCC in chronic hepatitis B. We extended our study including patients with chronic hepatitis $\mathrm{C}$ to see if liver volume maintains prognostic value in the development of viral hepatitis-associated HCC.

Methods : A total of 706 patients were included in this study : 429 with chronic hepatitis B (CHB) and 277 with chronic hepatitis C (CHC). HCC surveillance was done by periodic examination of liver ultrasound and serum alpha-fetoprotein. Liver volume was measured from the cross-sectional images of portal phase dynamic CT study, which was performed during the recall policy of HCC surveillance.

Results : Liver volume index, the ration of predicted-to-estimated liver volume is a significant predictor of HCC (hazard ratio [HR] = $10.2,95 \% \mathrm{CI}=5.6-18.4, \mathrm{p}<0.001$ ). The liver volume index predicted increased HCC risk both in CHB and CHC (HR=9.1 and 16.3, respectively). In multivariate Cox analysis, liver volume index remained significant along with male sex, liver cirrhosis, ald age high gamma glutamyltransferase and low platelet counts.

Conclusion : Liver volume add prognostic information to the conventional combination of predictors in the prediction of viral hepatitis-associated HCC.

\section{IP-94_H}

In vivo rapid genetic screen of efficacy biomarkers for multi-kinase inhibitors in Hepatocellular carcinoma

Department of Gastroenterology and Hepatology, Osaka University Graduate School of Medicine

OTakahiro Kodama, Yuta Myojin, Hayato Hikita, Ryotaro Sakamori, Tomohide Tatsumi, Tetsuo Takehara

Background : Response rates of molecular-targeted drugs for hepatocellular carcinoma (HCC) are unsatisfactory due to its high inter-tumor heterogeneity of genetic drivers. We have developed novel HCC model mimicking inter-tumor driver diversity and rapidly screened efficacy biomarkers of multi-kinase inhibitors.

Methods : Pooled library of 10 clinically-relevant HCC oncogene cDNAs flanked by unique barcode and PiggyBac (PB) transposon was administered into liver of wild-type mice by hydrodynamic tail vein injection, together with $\mathrm{PB}$ transposase, leading to their genomic transposition and constitutive activation of random oncogenes in liver cells.

Results : Mice developed multiple genetically heterogeneous liver tumors within 2 weeks after library administration. They were treated with lenvatinib or sorafenib and oncogene abundance in every tumor was quantified by high-throughput barcode sequence. Abundance of tumor cells expressing MET or NRAS was significantly lower in sorafenib-treated tumors compared to vehicle, while abundance expressing FGF19 was significantly lower in lenvatinibtreated tumors. IC50 values for lenvatinib were negatively correlated with FGF19 levels In vitro. FGF19 inhibition in FGF19amplified HCC cell lines, Hep3B and HuH7, significantly suppressed cell growth and mitigated the efficacy of lenvatinib. FGF19 levels were markedly downregulated in Hep3B clones acquiring lenvatinib-resistance.

Conclusion : Our genetic screen identified that tumor levels of MET and NRAS for sorafenib and FGF19 for lenvatinib could be potential efficacy biomarkers toward HCC.

HCC

FGF19

\section{IP-95_H} Comprehensive analysis of circulating microRNA as a predictive biomarker for lenvatinib therapy

Department of Gastroenterology \& Neurology, Faculty of Medicine, Kagawa University

OAsahiro Morishita, Mai Nakahara, Tsutomu Masaki

The prognosis of advanced HCC remains poor. Lenvatinib, an oral multikinase inhibitor, brought about additional options for advanced HCC therapy. Sorafenib and regorafenib were recently used for advanced HCC that has progressed on lenvatinib therapy. Therefore, predictive biomarker for lenvatinib therapy is required to switch lenvatinib to next anticancer drugs.

MicroRNAs have recently been evaluated as tissue biomarkers for the response of anti-cancer drugs. However, no biological marker in serum, including circulating microRNA, has yet been discovered for the prediction of refractory for lenvatinib therapy in HCC.

Lenvatinib inhibited the cell proliferation in Hep3B, and Huh7 cells (effective group ; EG), but not in PLC/PRF/5 and Li-7 cells (noneffective group; NEG). Sixty-five miRNAs were significantly changed in the medium of lenvatinib EG and NEG. In addition, lenvatinib resistant cells which we generated were analyzed and several miRNAs were commonly altered in the medium as compared to parental cells. MicroRNA analysis was also performed using serum samples of HCC patients between EG (CR/PR) and NEG (PD) before lenvatinib administration. Among in vitro and in vivo results, 3 microRNAs were commonly detected by microRNA array and highly up-regulated in EG as compared to those in NEG by real time RT-PCR.

Therefore, we elucidate specific circulating microRNAs involved in refractory for lenvatinib therapy. Identification of specific microRNAs may develop a new critical predictive biomarker for the efficacy of lenvatinib therapy in HCC. 
Liver Cancer Prevention Research Unit, RIKEN Center for Integrative Medical Sciences

OXian-Yang Qin, Soichi Kojima

Acyclic retinoid (ACR), a chemopreventive agent against hepatocellular carcinoma (HCC), selectively kills HCC progenitor cells. Here, we performed a genome-wide transcriptome screen and identified that ACR suppressed the expression of a proto-oncogene MYCN selectively expressed in HCC cells but not in normal hepatic cells. Data mining of clinical datasets showed that MYCN expression in HCC was correlated positively with both cancer stem cell (CSC) and Wnt/ $\beta$-catenin signaling markers, whereas negatively with mature hepatocyte markers. Immunofluorescence and flow cytometric analyses showed MYCN expression marked an EpCAM+ CSC-like subpopulation, which was selectively targeted by ACR. In a cohort study of 102 HCC patients in Japan, MYCN was expressed at higher levels in HCC tumor than in non-tumor tissues. There was a significant positive correlation between $M Y C N$ expression and recurrence of $\mathrm{HCC}$ with single tumor but not with multiple tumors. In a cohort study of 118 HCC patients from Taiwan Liver Cancer Network (TLCA), MYCN expression was 1) significantly higher in HCC tumor than in non-tumor tissues ; 2) significantly higher in HCC tumor in patients with recurrence than those without recurrence ; 3 ) positively correlated with the recurrence rate of HCC patients without cirrhosis or with single tumor. In summary, these results suggest that MYCN will be a prognostic biomarker for recurrence and early stage of HCC.

MYCN $\quad$ Liver cancer

IP-97_H $\begin{aligned} & \text { Clinical significance of circulating } \\ & \text { immuno-oncology checkpoint proteins in }\end{aligned}$ advanced HCC

Osaka City University Graduate School of Medicine Department of Hepatology

OPhuong Minh Dong, Masaru Enomoto, Le Thi Thanh Thuy, Hoang Hai, Atsushi Hagihara, Sawako Uchida-Kobayashi, Akihiro Tamori, Norifumi Kawada

Background : Clinical significance of circulating immuno-oncology checkpoint molecules as a predictor of patients'outcome or therapeutic response has been evaluated in various cancers, but not in hepatocellular carcinoma (HCC). Methods : Forty-eight patients with advanced HCC who received sorafenib (median age, 70 years ; 33 men and 15 women ; 23 in BCLC stage B and 25 in C) were included. Plasma samples were collected at baseline, 1 , and 2 weeks of treatment. The concentration of 16 soluble immunooncology proteins were measured using multiplexed fluorescent bead-based immunoassays. Results : Multivariate analysis showed that inhibitor factor sBTLA levels at baseline were an independent predictor of overall survival (HR, 2.144;95\% CI, 1.023-4.489; $\mathrm{p}=$ 0.043 ). After 1 week, only sCD28 was increased significantly ( $\mathrm{p}=$ 0.0197 ). Interestingly, at week 2 , ten of 16 proteins were significantly increased including sBTLA, sLAG-3, sTLR-2, sGITRL, sPD-1, sCTLA-4, sCD80, sCD86, sPD-L1, and sICOS. The positive correlations between fold changes of soluble receptors and their ligands were observed (ex. sCTLA4 vs sCD80/sCD86 and sPD-1 vs sPD-L $1 / \mathrm{sCD} 80$ ). Fold changes of sCTLA-4 and sPD-1, sPD-L1 were also positively correlated. Conclusion : sBTLA can be a good biomarker predicting the outcome in HCC patients. The dynamic changes of soluble immuno-oncology checkpoint proteins suggested that molecular-targeted therapy can affect the immune system of advanced HCC patients. 


\section{$\star$ IP-98_S}

The application of circulating tumor DNA analysis for detecting minimal residual disease and predicting recurrence in colorectal cancer patients

Department of Gastroenterological and Surgery, Cancer Institute Hospital, Japanese Foundation for Cancer Research ${ }^{11}$, Cancer Precision Medicine Center, Japanese Foundation for Cancer Research ${ }^{2)}$ OSatoshi Nagayama ${ }^{1)}$, Hiu Ting Chan ${ }^{2)}$, Siew-Kee Low ${ }^{2}$, Kazuma Kiyotani ${ }^{2}$, Tomohiro Yamaguchi ${ }^{1}$, Toshiya Nagasaki ${ }^{12}$, Takashi Akiyoshi' ${ }^{1 /}$, Tsuyoshi Konishi' ${ }^{1)}$ Yosuke Fukunaga ${ }^{1)}$, Yusuke Nakamura ${ }^{2}$, Masashi Ueno ${ }^{1)}$

We evaluated the feasibility of circulating tumor DNA analysis in detecting minimal residual disease in colorectal cancer (CRC) patients. Three sets of plasma, peripheral blood and tumor tissues were collected from 45 CRC patients. Genomic DNA was extracted from tumor tissue and peripheral blood that were used to evaluate the concordance between tissue and cell-free DNA/RNA (cfDNA/ RNA) and mutations derived from clonal haematopoiesis (CH). Next generation sequencing was performed by using Oncomine PanCancer Cell-Free Assay. Twenty-seven (69\%) of 39 pre-operative patients were found to have at least 2 copies of somatic mutations from cfDNA/RNA. Among the 39 patients that were monitored, one stage IIIb patient who carried BRAF V600E mutation with a MAF of $0.067 \%$ pre-operatively, showed 8 -fold increase $(0.530 \%)$ in $\mathrm{MAF}$ at the time of recurrence. The MAF of BRAF V600E was reduced to $0.1759 \%$ one month after surgical removal of the metastatic lesions. In another patient, GNAS R201C was repeatedly detected from pre- and post-operative cfDNA/RNA samples. Such mutation was also detected from the genomic DNA indicating this mutation is likely to be derived from $\mathrm{CH}$ and should be excluded as a monitoring marker.

\section{IP-100_S}

Clinical significance of tumor depth for rectal cancer with lateral lymph node involvement.

Department of Surgery, Coloproctology Center Takano Hospital OKazutsugu Iwamoto, Yasumitsu Saiki, Masafumi Tanaka, Mitsuko Fukunaga, Tadaaki Noguchi, Kazutaka Yamada

In our institute, total mesorectum excision (TME) with lateral lymph node dissection is recognized as standard surgical procedure for lower locally advanced rectal cancer. Patients and methods : 631 patients underwent potentially curative surgery for rectal cancers located (2001-2017) were reviewed in the present study. Results : Male patients were $65.5 \%$ and Female $34.5 \%$. Ra : $29.8 \%$, $\mathrm{Rb}: 62.7 \%, \mathrm{P}: 7.4 \%$. T1 tumors $: 21.4 \%$, T2 $: 22.3 \%$, T3 $: 41.2 \%$, T $4 \mathrm{a}: 9.0 \%$ and $\mathrm{T} 4 \mathrm{~b}: 6.0$ percent. Lateral nodal involvement (LNI) was observed in 72 patients $(11.4 \%)$. LNI was observed T2 tumor was $5.7 \%$, T3 $: 17.7 \%$, T4a : $9.6 \%$ and T4b : $40.7 \%$. (P<0.001). Patients who had nodal involvement in IMA area and SRA area and mesorectal area were observed $29.6 \%$. And 5 year Over all survival (5OS) were T2 : 92.1\%, T3 : 84.2\%, T4a : $65.5 \%$ and T4b 59.0\% (P $<0.001)$. A significantly increased incidence of positive lateral lymph node, 5 OS who had LNI were T2 : 83.3\%, T3 : 73.2\%, T4a : $37.5 \%$ and $\mathrm{T} 4 \mathrm{~b} 11.4 \%(\mathrm{P}<0.001)$. Conclusion : Poor prognostic risk factors of rectal cancer were doper tumor like T4a and T4b with Lateral Lymph Node Involvement. The findings a thorough preoperative examination of advanced rectal cancer such as an optimal size for lymph nodes and deeper depth, it will determine to add neoadjuvant chemoradiotherapy or chemotherapy. More research is needed to investigate this issue in greater depth.

rectal cancer

surgery

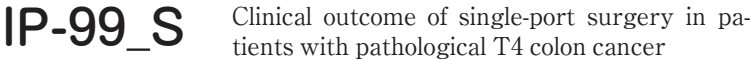

Department of Surgery, Osaka Rosai Hospital ${ }^{1)}$, Department of Surgery, Osaka Police Hospital ${ }^{2}$

OMitsuyoshi Tei ${ }^{1)}$, Toshinori Sueda ${ }^{1)}$, Haruna Furukawa ${ }^{1)}$, Tae Matsumura $^{1)}$, Chikato Koga ${ }^{1)}$, Masaki Wakasugi ${ }^{12}$, Ryohei Kawabata $^{1)}$, Jyunzo Shimizu ${ }^{1)}$, Junichi Hasegawa ${ }^{1)}$, Hiroki Aka$\mathrm{matsu}^{2)}$

【Purpose】 The aim of this study was to evaluate the clinical outcomes of single-port surgery (SPS), compared with those of multiport surgery (MPS) in patients with pathological T4 colon cancer. 【Methods】 A total of 188 patients with proven pathological T4 colon cancer who underwent laparoscopic surgery from January 2008 to December 2014 were enrolled in this study. These patients were divided into the SPS group $(n=94)$ and the MPS group ( $n=94)$. The clinical outcomes were compared between groups. 【Results】 The median operative time was significantly shorter in the SPS group than in the MPS group $(\mathrm{P}=0.045)$. Postoperative complications did not differ between the groups. The length of postoperative hospital stay was significantly shorter in the SPS group than in the MPS group $(\mathrm{P}<0.001)$. Oncological resection rates were similar between groups. The 3-year relapse-free survival rates, the 3-year local recurrence-free survival rates and the 5-year overall survival rates were similar between groups. [Conclusions] Single-port surgery is safe and feasible in patients with pathological T4 colon cancer.
IP-101_S

Clinical diagnosis and treatment of colorectal cancer using a gene-targeted panel test for next-generation sequencing

Department of Surgical Oncology, The University of Tokyo Kazuhito Sasaki, Keisuke Hata, Hiroaki Nozawa, Kazushige Kawai, Toshiaki Tanaka, Takeshi Nishikawa, Yasutaka Syuno, Soichiro Ishihara

Background : Next-generation sequencing (NGS) of tumor sample DNAs can guide clinical cancer management by providing diagnostic or prognostic data, and facilitating the identification of potential treatment regimens. We evaluated the efficacy of targeted sequencing for the management of colorectal cancer (CRC) in patients undergoing elective surgery.

Methods : Matched Formalin-fixed, paraffin-embedded (FFPE) tumor tissues and blood samples were obtained from eight patients with sporadic CRC who underwent elective surgery. Genomic DNA and RNA were extracted from FFPE sections. NGS was performed to investigate mutations in a panel of over 400 cancer-related genes.

Results : Our panel detected two BRAF, one RAS, one MAP3K1, and one BRCA2 mutations, one PTEN deletion, and two hypermutations. In one case, submucosal tumor like mass, which was initially thought to be lymph node metastasis, was diagnosed as primary colon cancer owing to variations in gene profiling results. Moreover, in this case, adjuvant chemotherapy with a platinum compound was provided because of the detection of BRCA2 mutation.

Conclusions : In this series, the genetic analysis contributed to the accurate diagnosis and selection of the adjuvant chemotherapy regimen in one patient. Identification of novel treatment targets and subsequent drug development using the cancer genome information will allow us to develop tailor-made medicine in the future. 

IP_102_S Gene panel testing detects actionable gecer

Division of Digestive and General Surgery, Niigata University Graduate School of Medical and Dental Sciences ${ }^{1)}$, Division of Pediatric Surgery, Niigata University Graduate School of Medical and Dental Sciences ${ }^{2}$, Department of Surgery, Niigata Cancer Center Hospital $^{3)}$

OYoshifumi Shimada ${ }^{1)}$, Masayuki Nagahashi ${ }^{1 /}$, Hiroshi

Ichikawa $^{1)}$, Yosuke Tajima ${ }^{1)}$, Kaoru Abe ${ }^{1)}$, Kana Tanaka ${ }^{1)}$,

Hidehito Oyanagi ${ }^{1)}$, Kizuki Yuza ${ }^{1)}$, Yuki Hirose ${ }^{1)}$, Kohei Miura ${ }^{1)}$,

Takaaki Hanyu ${ }^{1)}$, Kazuyasu Takizawa ${ }^{1)}$, Masato Nakano ${ }^{1)}$, Jun

Sakata $^{1)}$, Takashi Kobayashi ${ }^{1.2}$, Hitoshi Kameyama ${ }^{1)}$, Hitoshi

Nogami $^{3)}$, Satoshi Maruyama ${ }^{3)}$, Yasumasa Takii ${ }^{33}$, Toshifumi

Wakai $^{1)}$

BACKGROUNDS : Right-sided colorectal cancer (RCRC) has different tumor biology from let-sided CRC (LCRC), and RCRC showed worse prognosis and resistance to anti-EGFR therapy compared with LCRC. We aimed to identify actionable genetic alterations according to primary tumor sidedness.

MATERIALS AND METHODS : Two-hundred one Japanese patients with Stage I-IV CRC were analyzed. We retrospectively investigated genetic alterations using a 415-gene panel testing, and evaluated actionable genetic alterations in RCRC.

RESULTS : Mutations in KRAS, PIK3CA, RNF43, BRAF, ACVR2 A, MSH6, and PALB2 were significantly associated with RCRC. Microsatellite instability-high and $B R A F$ V600E mutation were significantly associated with RCRC $(P=0.028$ and $P<0.001$, respectively). RCRC had significantly more genetic alterations associated with resistance to anti-EGFR therapy (TK receptors. RAS and PI3 K pathway) compared with LCRC $(P=0.009)$.

CONCLUSION : RCRC is more likely to harbor genetic alterations associated with response to immunotherapy and anti-BRAF therapy, while RCRC is more likely to harbor genetic alterations associated with resistance to anti-EGFR therapy.

right-sided colorectal cancer genetic alteration 


\section{QIP-103_S}

Prognosticators of Long-term Outcomes of TNM stage II Colorectal Cancer : Molecular Patterns or Clinicopathological Features

Taipei Veterans General Hospital

OTai-Chuan Kuan, Shih-Ching Chang, Jen-Kou Lin, Shung-Haur Yang, Jeng-Kae Jiang, Wei-Shone Chen, Tzu-Chen Lin, HuannSheng Wang, Yuan-Tzu Lan, Chun-Chi Lin

\section{Background :}

Patients with stage II colorectal cancer (CRC) have a higher risk of recurrence when certain risk factors present. However, the prognostic role of molecular patterns for stage II disease is still unclear.

\section{Methods :}

A total of 509 patients were enrolled in this study, and all clinical, pathological, and molecular data were collected. Molecular patterns pathological, and molecular data were collected. Molecular patterns included microsatellite instability, elevated microsatellite altera-
tions at selected tetranucleotides (EMAST) status, KRAS, BRAF, tions at selected tetranucleotides (EMAST) status, KRAS, BRAF,
APC, TP53, PI3K, AKT1, and other gene mutations. The endpoints APC, TP53, PI3K, AKT1, and other gene mutations. The endpoints were oncological outcomes, including overall survival (OS), cancer-
specific survival (CSS), disease-free survival (DFS), local recurrence (LR), and distant recurrence (DR). Cox regression method was used.

\section{Results :}

Numerous molecular patterns influenced the oncological outcomes in univariate analysis, but no variable reached significance in LR. In multivariate analysis, mucinous component (MC) $>50 \%(\mathrm{P}<0.01)$ was crucial for OS and CSS. Lymphovascular invasion (LVI) (P< $0.01), \mathrm{MC}>50 \%(\mathrm{P}<0.01)$, and EMAST-H $(\mathrm{P}=0.02)$ significantly affected DFS, whereas LVI $(\mathrm{P}<0.01)$, MC $>50 \%(\mathrm{P}<0.01)$, and TP 53 mutation $(\mathrm{P}=0.02)$ were crucial for $\mathrm{DR}$

\section{Conclusions :}

In this study, microsatellite instability, EMAST, KRAS, and BRAF alterations do not influence OS, CSS, DFS for stage II CRC. Overall, LVI and MC are two significant prognostic factors for DFS and DR.

\section{IP-104_S}

Exosomal Protein Biomarkers in Plasma Acquired by Absolute Quantitative Proteomic Analysis for Colorectal Cancer

Department of Gastrointestinal Surgery, Kyoto University ${ }^{1)}$, Laboratory of Proteome Research, National Institutes of Biomedical Innovation, Health and Nutrition ${ }^{2}$, Gastroenterological Center, Department of Gastroenterological Surgery, Cancer Institute Ariake Hospital, Japanese Foundation for Cancer Research ${ }^{3}$

OKeiko Kasahara ${ }^{1,2)}$, Satoshi Nagayama ${ }^{3)}$, Ryohei Narumi ${ }^{2)}$,

Takeshi Tomonaga ${ }^{2)}$, Jun Adachi ${ }^{2}$, Yoshiharu Sakai ${ }^{1)}$

[Introduction] Selected reaction monitoring $(\mathrm{SRM})$ is a reliable proteomic analysis to enable absolute quantitative measurement with high reproducibility.[Materials \& Methods] $100 \mu \mathrm{l}$ of aliquots of plasma were processed by using ultracentrifugation. Plasma samples were obtained for 59 patients with colorectal cancer (Stage I $\mathrm{N}=26$, Stage IV N=33) and 59 healthy volunteers. SRM assay was performed. A bioinformatics approach supported the selection of proteins as biomarkers. [Results \& Conclusion] 1,344 peptides corresponding 450 proteins were listed as biomarker candidates and measured. 70 proteins were selected with the result of prediction methods from statistics and machine learning. We will show you the deliberate strategy for biomarker discovery.

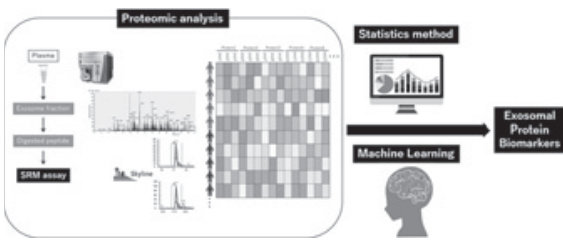

Exosome

Absolute Quantitative Proteomic Analysis
Q

Journey for patients following ileostomy creation is not straightforward

Division of Colorectal Surgery, National University Hospital ODedrick Kok Hong Chan, Ker-Kan Tan

Background

An ileostomy is usually created to avert systemic sepsis in a patient with a tenuous anastomosis. However, what is often not reported are the numerous issues facing these patients subsequently, ranging from readmissions, non-reversal of the stoma and complications from the closure.

Methods

We conducted a retrospective analysis of patients who had an ileostomy created from 2011 to 2016 . Statistical analysis was performed to identify risk factors for readmissions and ileostomy nonreversal.

Results

193 patients had an ileostomy created during the study period. Twenty-six (13.5\%) patients developed stoma related complications requiring readmission. The most common cause of readmission $(9.3 \%)$ was due to dehydration and acute kidney injury secondary to high stoma output.

One hundred and thirty (67.4\%) patients had their ileostomy reversed. On multivariate analysis, only stomas created during an ultralow anterior resection were associated with reversal (OR2.88 $(95 \% \mathrm{CI}, 1.24-6.68) ; \mathrm{p}=0.014)$. There was a trend towards significance for permanent ileostomies in patients age $>50$ (OR0.358 (95\% CI,0.120-1.07) ; $\mathrm{p}=0.065$ )

Among patients who underwent ileostomy reversal, seven $(3.6 \%)$ developed complications. Four $(2.1 \%)$ suffered from an anastomotic leak requiring surgical intervention with one mortality from sepsis. Two (1.0\%) patients were readmitted for bleeding while one $(1.4 \%)$ developed a wound infection requiring antibiotics. Conclusion

Almost half of the patients who had an ileostomy had an undesirable outcome following closure. Patients need to be properly counselled about the risks involved prior to the index operation.

ileostomy

complications

QIP-106_S

Are we resecting too much? Having 5 $\mathrm{cm}$ surgical margins are unecessary to reduce local recurrence in colon cancer.

University Surgical Cluster, National University Health System, Singapore

OKai Yin Lee, Frederick Hong Xiang Koh, Dedrick Chan, Ker Kan Tan

Current clinical guidelines recommend surgical resection margins of at least $5-7 \mathrm{~cm}$ proximally and distally in the treatment of colon cancer. Resection margins have been well studied in rectal cancer, but remains lacking in colon cancer. The objective of the study is to investigate the role of resection margins on local recurrence in colon cancer.

A retrospective study of all patients diagnosed with colorectal cancer from 2008-2013 was conducted. Patients managed with palliative intent, rectal tumours, non-adenocarcinoma tumours were excluded. We compared patients with resection margins of at least 5 $\mathrm{cm}$ and less than $5 \mathrm{~cm}$. Univariate, multivariate and survival analysis were performed.

251 patients were analysed over a median follow up of 57 months (range 0-101 months). 175 (69.7\%) patients had either resection margins not meeting $5 \mathrm{~cm}$. There were $23(13.1 \%)$ local recurrences with median time to recurrence of 21.5months (range 3-102 months). Using a cutoff of $5 \mathrm{~cm}$, we showed no significant differences in local recurrence for both proximal and distal margins. T4 tumours, rectosigmoid tumours and histological pedicle invasion are independently associated with local recurrence.

Current cutoffs of $5 \mathrm{~cm}$ for surgical resection margins in colon cancer is not associated with increased risk of local recurrence. Smaller margins of up to $4 \mathrm{~cm}$ may potentially be safely taken without affecting risk of locoregional recurrence nor pericolic lymph node yield.

Colon cancer

Resection margins 

IP-107_S $\begin{aligned} & \text { Oxaliplatin Based Chemotherapy Followed } \\ & \text { by Surgery for Clinical Stage II/III Lower }\end{aligned}$ Rectal Cancer

Department of Gastroenterological Surgery, Osaka University Graduate School of Medicine ${ }^{1)}$, Department of Surgery and Science, Kyushu University Graduate School of Medicine ${ }^{2}$

$\bigcirc$ Hidekazu Takahashi ${ }^{1}$, Norikatsu Miyoshi ${ }^{1}$, Naotsugu Haraguchi $^{12}$, Taishi Hata ${ }^{1)}$, Chu Matsuda ${ }^{1)}$, Hirofumi Yamamoto ${ }^{1)}$, Tsunekazu Mizushima ${ }^{1)}$, Masaki Mori', Yuichiro Doki ${ }^{12}$

[Background] Overall survival rate was not improved by neoadjuvant radio-chemotherapy, therefore for neoadjuvant therapy, chemotherapy with adequate intensity for distant metastasis is thought to be ideal. We have been adopted oxaliplatin-based neoadjuvant chemotherapy (NAC), prior to TME+LLND, and postoperative adjuvant chemotherapy against lower rectal cancer patients with T3 or deeper under the hypothesis of that NAC should have effect on primary lesion as well as distant metastasis. Here, we report on the completion rate and mid-term outcomes of cases treated with TME+LLND combined with pre- and postoperative chemotherapy.[Patients and Methods] From 2011 to 2018, 50 patients were enrolled. Male/female ratio $31: 19$, age was $19-76$ (median 64) years old.[Results] Oxaliplatin based regimen was selected as NAC. Doublet/Triplet ratio, $27: 23$. The completion rate of NAC was $44 / 50(88.0 \%)$. All other cases $3-5$ courses of XELOX therapy were adjuvanted with adjuvant chemotherapy. Recurrence was confirmed in 11 case, two patients died from rectal cancer (5-year recurrence free survival:71.0\%, 5-year survival rate: $92.4 \%$, follow-up period 975 days), and there was no difference in longterm oncological outcomes between doublet/triplet NAC regimen in this analysis. [Summary] Neoadjuvant chemotherapy for locally advanced rectal cancer was feasible.

advanced rectal cancer

neoadjuvant chemotherapy 


\section{IP-108_S}

Low platelet recovery predicts delayed functional and volume recovery after liver resection

Department of Digestive Surgery, Nihon University School of Medicine

OShintaro Yamazaki, Yusuke Mitsuka, Nao Yoshida, Tokio Higaki, Tadatoshi Takayama

Background : This study analyzed the relationship between platelet recovery and post-resection liver regeneration.

Methods : 343 consecutive patients with liver resection $>2$ Couinaud's segments were analyzed. Patients were divided into groups of delayed or normal platelet recovery rate on the basis of platelet count on postoperative day (POD) 7. Data were matched before analysis to adjust for operation scale. Trends in liver functional, liver volume recovery and remnant ischemic area were calculated by $\mathrm{CT}$ volumetry. Factors predicting liver regeneration were assessed.

Results : In 78 matched-pair patients, all-complications rate $(42.3 \%$ vs. $26.9 \%, p=0.002)$ and infectious complications rate $(21.8 \%$ vs. $9.0 \%, \mathrm{p}=0.027$ ) were significantly higher in the low platelet recovery group. Liver function did not differ significantly, while significant differences remained for platelet recovery in all patients $(\mathrm{p}<$ $0.01)$ and patients with colorectal metastasis $(\mathrm{p}<0.01)$. Parenchyma volume recovery was delayed in the low platelet recovery group from POD7 ( $84.5 \%$ vs. $78.1, \mathrm{p}<0.01)$ to POD30 (92.5\% vs. 85.6, p $<0.01$ ). Platelet recovery rate on POD7 correlated negatively with ischemic liver volume as evaluated on POD2 by CT $(\mathrm{r}=0.691)$. Postoperative ischemic volume was highest on POD2 (5.41 [1.98-11.21] $\mathrm{p}<0.001$ ), and infectious complications (3.48 [1.44-7.37], $\mathrm{p}<$ $0.001)$ and multiple resection (1.67 [1.10-4.11], $\mathrm{p}=0.011)$ predicted low platelet recovery on multivariate analysis.

Conclusion : Platelet recovery predicted liver functional and volume recovery, and occurrence of complications.

\section{IP-109_S}

Liver Transplantation for the Curative Treatment of Secondary Biliary Cirrhosis Caused by Hepatolithiasis : A Case Series

\section{Changhua Christian Hospital}

$\bigcirc$ LiZzu Lin

Hepatolithiasis is a calculus disease of liver with bile stone within the intrahepatic biliary ducts. The prevalence is rare in Western societies, but relative high in East Asia. Mostly, hepatolithiasis complicated with pyogenic cholangitis, liver abscess or even sepsis. Initially, it belongs a benign disease. However, due to the risk of secondary biliary cirrhosis and malignant transformation, aggressive intervention includes surgical and non-surgical treatments are necessary. In this study, we presented four cases of secondary biliary cirrhosis caused by recurrent intra-hepatic cholangiolithiasis were treated by liver transplantation. One patient received deceased donor liver transplant (DDLT), and the other three received living donor liver transplantation (LDLT). Under 51 months of median follow up period, no recurrence of hepatolithiasis was found.
IP-110_S

The Inflammatory Biomarkers in Hepatocellular Carcinoma Following Hepatic Resection : Prognostic Significance and Association with Programmed Death Ligand 1 Expression

Department of Surgery and Science, Kyushu University OShinji Itoh, Tomoharu Yoshizumi, Kyohei Yugawa, Takeshi Kurihara, Shohei Yoshiya, Yohei Mano, Kazuki Takeishi, Noboru Harada, Toru Ikegami, Yuji Soejima, Masaki Mori

Background : We investigated the prognostic value of the inflammatory biomarkers, neutrophil-to-lymphocyte ratio (NLR), plateletto-lymphocyte ratio (PLR) and lymphocyte-to-monocyte ratio (LMR) in patients with hepatocellular carcinoma (HCC) undergoing curative hepatic resection and their associations with programmed death ligand 1 (PD-L1) expression, and tumor microenvironment were further explored. Method : A total 428 patients who underwent hepatic resection for HCC were retrospectively analyzed. The impact of inflammatory biomarkers on overall survival (OS) and recurrence-free survival (RFS) was assessed. Immunohistochemical examination using PD-L1, CD8 and CD68 stainings were performed. HCC cell lines were evaluated for PD-L1 expression. Results : Multivariate analysis showed that a low LMR was an independent prognostic factor for OS and RFS after hepatic resection for HCC, and the NLR and PLR were not independently significant. LMR was significantly lower in the PD-L1-positive patients compare with the PD-L1-negative patients. Consistent results in the validation cohort strengthened the prognostic value of LMR and association between LMR and PD-L1 expression. PD-L1 expression was upregulated in HCC cell lines treatment with interferon- $\gamma$ and co-cultured with monocyte cells (THP-1).Conclusion : Our results show that LMR is an independent predictor of survival after hepatic resection in patients with HCC. Activation of the immune checkpoint pathwayin the tumormicroenvironmentis associated with a low LMR.

Hepatocellular carcinoma immune checkpoint

\section{IP-111_S Genomic sequencing in HBP malignancies}

Department of HBP Surgery, Tokyo Medical and Dental University $^{1)}$, Department of Molecular Oncology, Tokyo Medical and Dental University ${ }^{2}$, Cancer Center, Tokyo Medical and Dental University $^{3)}$

OHiroaki Ono ${ }^{1)}$, Sadakatsu Ikeda ${ }^{3)}$, Kosuke Ogawa ${ }^{1)}$, Toshiro Ogura $^{1)}$, Daisuke Ban ${ }^{1)}$, Atushi Kudo ${ }^{1)}$, Satoshi Miyake ${ }^{3)}$, Shinji Tanaka ${ }^{2)}$ Minoru Tanabe ${ }^{1)}$

Background : Genomic sequencing is a comprehensive method to investigate tumor genomic mutation by next generation sequencer. Whole-genomic analysis can figure out actionable tumor mutation and lead to individual suitable treatment matching tumor genomic information.

Methods : Our university have started the comprehensive genomic sequencing analysis since August 2017. Total 90 patients have utilized sequencing analysis using panels of Gurdant360, OncoPrime Basic, or OCA v3, until November 2018 .

Results : 39 patients were carried out in our department. They were 18 patients of neuroendocrine tumor (NET) including gastrointestinal NET most frequently, 17 of pancreatic cancer, and 5 of biliary tract cancer and so on. In the patients of NET, actionable mutation, which might be related to treatment, was harbored in 13 cases $(76.5 \%: 13 / 17)$. As result, novel treatments indicated by genomic sequencing were started in 3 patients $(17.6 \%: 3 / 17)$. Concretely, BRCA1 mutation, which is associated with DNA damage repair system, was harbored in one patient. And CDKN2A mutation, which is associated with cell cycle regulation, was harbored in one patient. Another patient was diagnosed as high tumor mutation burden which was indicative for immune checkpoint inhibitor.

Conclusion : Precision medicine can lead to order-made therapy so as to explore treatments for HBP malignancies.

Pancreas

Genomic sequencing 
IP-112_S

Exosomes transfer information of cancer cells ; reprogramming of nonmetastasizing tumor cells and therapeutic efficacy

Department of Surgery, Surgical Oncology and Science, Sapporo Medical University ${ }^{11}$, Tumor Cell Biology, University Hospital of Surgery, Heidelberg ${ }^{2)}$, Department of Cell Science, Research Institute of Frontier Medicine, Sapporo Medical University School of Medicine $^{3)}$

ODaisuke Kyuno ${ }^{1)}$, Margot Zöller ${ }^{2}$, Takashi Kojima ${ }^{3)}$, Hiroshi Yamaguci $^{1)}$, Masafumi Imamura ${ }^{1 /}$, Minoru Nagayama ${ }^{1)}$, Kenji Okita $^{1)}$, Takayuki Nobuoka ${ }^{1)}$ Y Yasutoshi Kimura ${ }^{1)}$, Ichiro Take$\operatorname{masa}^{1)}$

Background : Exosomes function in the transport of biological information between all kinds of cells including tumor cells. We investigated whether cancer initiating cells (CICs) could transfer the information to non-CICs and whether we could use exosomes as therapeutic tools against CICs.

Method : Claudin7 is a CIC marker in gastrointestinal tumors and we used claudin7-rich and -knockdown pancreatic cancer cells as $\mathrm{CICs}$ and non-CICs, respectively. We co-cultured non-CICs with exosomes derived from CICs. We also transfected the anti-claudin7 microRNA to exosomes from fibroblasts and co-cultured the transfected exosomes with CICs.

Results : CIC-exosomes promoted tumor cell dissemination and metastatic growth. Migration, invasion, and angiogenesis of non-CICs were stron were was efficiently transferred into tumor cells. Claudin7-silencing was accompanied by reduced expression of additional cancer-initiating cell markers. The exosome-miRNA transfer provoked reduced anchorage-independent growth, loss in motility and invasiveness and mitigated apoptosis resistance of CICs.

Conclusion : These findings suggest exosomes could affect the invasiveness and metastatic capacity of tumor cells and could also be used as an efficient drug delivery system to suppress malignancy of tumor cells. cancer

\section{IP-113_S}

High-Grade PanIN and EMT-Associated Transcription Factors Predict Prognosis of Pancreatic cancer: New Perspective and Subgroups

Department of Molecular Pathology, Tohoku University School of Medicine $^{1)}$, Department of Surgery, Iwate Prefectural Chubu Hospital $^{2)}$, Department of Surgery, Tohoku University Hospital ${ }^{3)}$

OSho Fujiwara ${ }^{1.2)}$, Yuriko Saiki ${ }^{11}$, Shinichi Fukushige ${ }^{1 /}$, Masaharu Ishida ${ }^{3)}$, Fuyuhiko Motoi ${ }^{3)}$, Michiaki Unno ${ }^{3)}$, Akira Horii ${ }^{1)}$

\section{Backgrounds :}

Pancreatic ductal adenocarcinoma (PDAC) is the most lethal cancer. because of its highly invasive and metastatic nature. Most patients are diagnosed at an advanced stage with these malignant characteristics. Epithelial-to-mesenchymal transition (EMT) is known to play an important role for these malignant behaviors. High-grade pancreatic intraepithelial neoplasia (HG-PanIN) is known to be one of the major precursor lesions of PDAC. However, association with HG-PanIN and major EMT transcription factors has not been clearly understood.

Methods :

Surgically resected specimens from 71 PDAC patients are investigated and expressions of SNAIL, SLUG, TWIST1 and ZEB1 were immunohistochemically analyzed in PDAC and their adjacent HGPanIN. We analyzed their clinicopathological features and clinical outcomes.

\section{Results :}

Expressions of SNAIL and SLUG significantly increased in PDAC compared with HG-PanIN $(P<0.001$ and $<0.002)$. Especially, large tumor size and perineural invasion were more observed in PDAC accompanied by SNAIL-positive HG-PanIN group than -negative group ; $P=0.025$ and $P=0.036$, respectively. However, the PDAC accompanied by SNAIL-positive HG-PanIN group showed a significantly better relapse-free survival $(P<0.001)$ and overall survival $(P<0.001)$

\section{Conclusions :}

Our present results suggest existence of a distinct subgroup for precursor region of PDAC : SNAIL-positive HG-PanIN group.

\section{IP-114_S}

Initial Recurrence Pattern after Resection for Pancreatic Cancer : A Systematic Review and Meta-analysis

Department of General, Visceral and Transplantation Surgery, University of Heidelberg

Masayuki Tanaka, Andre Mihaljevic, Pascal Probst, Max Heckler, Ulla Klaiber, Ulrike Heger, Markus Buchler, Thilo Hackert

ABSTRACTBackground : Initial recurrence mapping of resected pancreatic ductal adenocarcinoma (PDAC) could help in stratifying patient subpopulations for optimal postoperative follow-up. The aim of this systematic review and meta-analysis was to investigate the initial recurrence patterns of PDAC and to correlate them with clinicopathological factors. Methods : The MEDLINE and Web of Science databases were systematically searched for studies reporting first recurrence patterns after PDAC resection. Data were extracted from the studies selected for inclusion. Pooled odds ratios (OR) and their $95 \%$ confidence intervals (CI) were calculated to determine the clinicopathological factors related to the recurrence sites. Results : Eighty-nine studies with 17,230 patients undergoing PDAC resection were included. The weighted median rates of initial recurrence sites were $20.8 \%$ for locoregional, $26.5 \%$ for liver, $11.4 \%$ for lung, and $13.5 \%$ for peritoneal dissemination. The weighted median overall survivals were 19.8 months (locoregional), 15.0 months (liver), 30.4 months (lung), and 14.1 months (peritoneal dissemination). Meta-analysis revealed that R1 (direct) resection, perineural invasion and positive peritoneal lavage cytology were significantly associated with peritoneal dissemination as initial recurrence site. Conclusion : Based on these stratifications and risk factors for initial recurrence patterns, correctly predicting the site and prognosis could be considered in decision-making for specific surveillance and treatment.

pancreatic cancer

recurrence pattern
IP_115_S Tumor growth rate could be a predictor of

Department of Surgery, Japanese Red Cross Kumamoto Hospital ${ }^{12}$, Department of Pathology, Japanese Red Cross Kumamoto Hospital ${ }^{2}$ $\mathrm{OKenji} \mathrm{Okumura}^{1)}$, Yasuhiro Kihara ${ }^{1)}$, Michiko Nagamine ${ }^{2)}$, Hiroshi Yokomizo

Introduction: Aggressive pancreatic cancer grows rapidly and often cannot be resected due to extensive invasion or dissemination in the operation. We hypothesized rapid growing pancreatic cancer would be aggressive and associate with worse outcomes. The aim of this study was to validate the tumor growth rate as a prognostic factor in pancreatic cancer.

Materials and Methods : We performed retrospective analysis of 92 patients with resected pancreatic caner in our institution and analyzed clinical characteristics and outcomes of the patients. Tumor growth rate was defined by $\{$ (pathological volume)-(CT volume) $\}$ / (interval days). We excluded patients with multiple cancers, neoadjuvant therapy or missing records.

Results : Median (IQR) age was 70 (60-77), male : female was 1 : 1. Median (IQR) Interval days between initial CT scan to operation was 19 (14-32). Five-year overall survival was $29 \%$. Large CT volume, large pathological volume and high tumor growth rate were significantly associated with worse outcomes $(\mathrm{p}<0.05)$. In other clinicopathological factors, pancreaticoduodenectomy, perineural invasion and lymphatic invasion were associated with worse outcomes $(\mathrm{p}<0.05)$. In multivariate analysis, tumor growth rate was a significant prognostic factor to predict five-year survival $(\mathrm{p}=0.004)$.

Conclusion : Our study showed rapid tumor growth rate was significantly associated with worse five-year overall survival. This study result supports our hypothesis and tumor growth rate could be a predictor of aggressiveness.

Pancreatic Cancer

Prognosis

HG-PanIN 